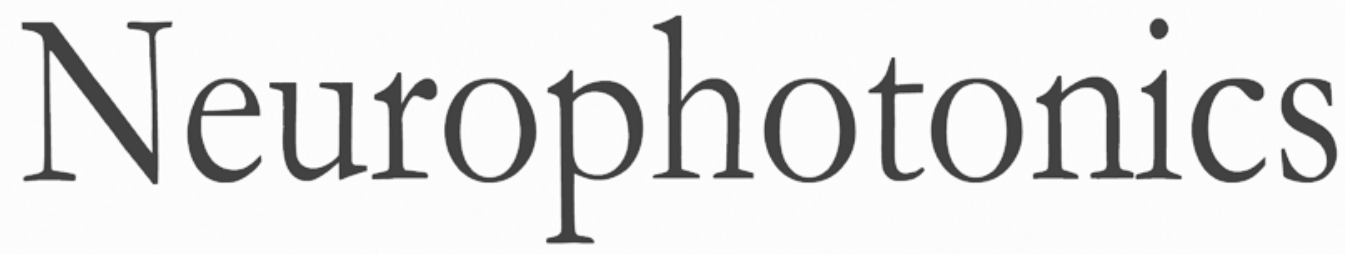

\title{
Cerebral blood flow and autoregulation: current measurement techniques and prospects for noninvasive optical methods
}

Sergio Fantini

Angelo Sassaroli

Kristen T. Tgavalekos

Joshua Kornbluth 


\title{
Cerebral blood flow and autoregulation: current measurement techniques and prospects for noninvasive optical methods
}

\author{
Sergio Fantini, ${ }^{\mathrm{a}, \star}$ Angelo Sassarolii, ${ }^{a}$ Kristen T. Tgavalekos, ${ }^{\mathrm{a}}$ and Joshua Kornbluth ${ }^{\mathrm{b}}$ \\ ${ }^{a}$ Tufts University, Department of Biomedical Engineering, 4 Colby Street, Medford, Massachusetts 02155, United States \\ ${ }^{\mathrm{b}}$ Tufts University School of Medicine, Department of Neurology, Division of Neurocritical Care, 800 Washington Street, Box \#314, Boston, \\ Massachusetts 02111, United States
}

\begin{abstract}
Cerebral blood flow (CBF) and cerebral autoregulation (CA) are critically important to maintain proper brain perfusion and supply the brain with the necessary oxygen and energy substrates. Adequate brain perfusion is required to support normal brain function, to achieve successful aging, and to navigate acute and chronic medical conditions. We review the general principles of CBF measurements and the current techniques to measure CBF based on direct intravascular measurements, nuclear medicine, X-ray imaging, magnetic resonance imaging, ultrasound techniques, thermal diffusion, and optical methods. We also review techniques for arterial blood pressure measurements as well as theoretical and experimental methods for the assessment of CA, including recent approaches based on optical techniques. The assessment of cerebral perfusion in the clinical practice is also presented. The comprehensive description of principles, methods, and clinical requirements of $\mathrm{CBF}$ and CA measurements highlights the potentially important role that noninvasive optical methods can play in the assessment of neurovascular health. In fact, optical techniques have the ability to provide a noninvasive, quantitative, and continuous monitor of CBF and autoregulation. ๑ 2016 Society of Photo-Optical Instrumentation Engineers (SPIE) [DOI: 10.1117/1.NPh.3.3.031411]
\end{abstract}

Keywords: Cerebral perfusion; autoregulation; computed tomography perfusion; perfusion magnetic resonance imaging; transcranial Doppler; laser Doppler flowmetry; near-infrared spectroscopy; diffuse correlation spectroscopy; coherent hemodynamics spectroscopy.

Paper 16001SSVR received Jan. 4, 2016; accepted for publication May 10, 2016; published online Jun. 21, 2016.

\section{Cerebral blood flow}

\subsection{Physiological importance and normal values of cerebral blood flow in adult humans}

The human brain is an organ with high-energy density demands, amounting to only $2 \%$ of the entire body mass (or $\sim 1.4 \mathrm{~kg}$ ) but accounting for about $20 \%$ of the total power consumption of a normal adult at rest (or $\sim 20 \mathrm{~W}$ ). Blood perfusion is responsible for the delivery of oxygen, which is necessary for the neuronal oxidative metabolism of energy substrates (mostly glucose, but also ketone bodies and lactate ${ }^{1}$ ). Because of the limited capacity of neurons for anaerobic metabolism (at rest, up to $92 \%$ of the adenosine triphosphate in the brain results from oxidative metabolism of glucose ${ }^{2}$ ), cerebral blood flow (CBF) is critically important for brain function and viability. It ensures proper delivery of oxygen and energy substrates and the removal of waste products of metabolism. Both hypoperfusion (insufficient $\mathrm{CBF}$ ) and hyperperfusion (excessive $\mathrm{CBF}$ ) can cause brain damage through ischemic injury, the former, and the breakdown of the blood-brain barrier, the latter, which can cause seizures, headaches, encephalopathy, and both ischemic and hemorrhagic stroke. ${ }^{3}$

$\mathrm{CBF}$ is defined as the blood volume that flows per unit mass per unit time in brain tissue and is typically expressed in units of $\mathrm{ml}_{\text {blood }} /\left(100 \mathrm{~g}_{\text {tissue }} \mathrm{min}\right)$. Alternatively, one may express $\mathrm{CBF}$ in terms of flow per unit volume of brain tissue, thus in

\footnotetext{
*Address all correspondence to: Sergio Fantini, E-mail: sergio.fantini@ tufts.edu
}

$\mathrm{ml}_{\text {blood }} /\left(100 \mathrm{ml}_{\text {tissue }} \mathrm{min}\right)$. The numerical values of CBF in the two cases differ by a factor given by the density of human brain tissue, which is about 1.04 to $1.06 \mathrm{~g} / \mathrm{ml}$ (with reported values, measured ex vivo, as high as $1.08 \mathrm{~g} / \mathrm{ml}) .{ }^{4}$ The normal average cerebral blood flow (CBF) in adult humans is about $50 \mathrm{ml} /(100 \mathrm{~g} \mathrm{~min}),{ }^{5}$ with lower values in the white matter $[\sim 20 \mathrm{ml} /(100 \mathrm{~g} \mathrm{~min})]$ and greater values in the gray matter $[\sim 80 \mathrm{ml} /(100 \mathrm{~g} \mathrm{~min})]^{2}$

\subsection{Factors that affect cerebral blood flow}

In the spirit of Ohm's law or Darcy's law, blood flow (BF) through a vascular segment can be expressed as the ratio between the pressure difference across that segment $(\delta P)$ and its vascular resistance $(R)$. Poiseuille's law expresses this resistance of a vascular segment $(R)$ in terms of its radius $(r)$, length $(L)$, and the blood viscosity ( $\eta$, usually expressed in centipoise, with $1 \mathrm{cP}=1 \mathrm{mPas}): R=8 \eta L /\left(\pi r^{4}\right)$. Even though $\mathrm{BF}$ does not strictly fulfill all requirements for the validity of Poiseuille's law (mostly because blood does not behave as a Newtonian fluid, especially in the microvasculature, blood vessels are not rigid pipes, and the flow velocity profile may deviate from the parabolic shape of steady laminar flow, especially at branching points or curved sections), it is nevertheless useful referring to it to appreciate, at least qualitatively, the factors that affect CBF. According to Poiseuille's law, the blood flow (BF, in units of blood volume per unit time) through a vascular segment of length $L$ and radius $r$, driven by a pressure difference $\delta P$, is given by 
$\mathrm{BF}=\frac{\delta P}{R}=\frac{\delta P \pi r^{4}}{8 \eta L}$

In the case of $\mathrm{CBF}$, the driving pressure is the so-called cerebral perfusion pressure (CPP), defined in the next paragraph, and the resistance is a total cerebrovascular resistance (CVR), which is associated with the entire brain vascular tree. The main sources of CVR are small arteries and pial arterioles, which can regulate their radius $(r)$ through vasodilatation and vasoconstriction.

The CPP is defined as the difference between the mean arterial pressure (MAP), which is the weighted average of the systolic and diastolic pressure, and the intracranial pressure (ICP), which is the pressure of the cerebrospinal fluid (CSF) in the subarachnoid space. The normal range for resting MAP is 70 to $100 \mathrm{mmHg}$ and for ICP it is 5 to $15 \mathrm{mmHg}$. From Eq. (1), it is apparent that changes in perfusion pressure, changes in vascular radius (i.e., vasodilation and vasoconstriction), and changes in blood viscosity all affect the CBF. Changes in perfusion pressure may occur under normal conditions, e.g., during a change in posture or exercise, or they may result from the administration of drugs or from pathological conditions such as subarachnoid hemorrhage (SAH), traumatic brain injury (TBI), and stroke. Blood viscosity is directly related to hematocrit and the concentration of hemoglobin in blood. While lower hematocrit decreases viscosity, thus increasing CBF according to Eq. (1), it also reduces the oxygen-carrying capacity of blood. The effect of the vascular radius $r$ on CBF is of particular interest because it is responsible for the modulation and regulation of $\mathrm{CBF}$, which is highly sensitive to $r$ as indicated by the fourth power of $r$ in Eq. (1), and we consider it next.

There are a number of factors that affect the vascular smooth muscles of small arteries and arterioles, resulting in their constriction or dilation. For example, carbon dioxide $\left(\mathrm{CO}_{2}\right)$ is a powerful vasodilator, so that $\mathrm{CBF}$ increases during hypercapnic conditions. Two processes that are of paramount importance in cerebral hemodynamics are the cerebrovascular responses to brain metabolism (neurovascular coupling) and to changes in perfusion pressure (CA).

Neurovascular coupling is responsible for the increase in $\mathrm{CBF}$ to support greater regional or global metabolic demands of the brain. This metabolism-driven increase in CBF is thought to be effected by a number of vasoactive mediators such as ions $\left(\mathrm{K}^{+}, \mathrm{H}^{+}, \mathrm{Ca}^{2+}\right)$, metabolic by-products (lactate, $\mathrm{CO}_{2}$, hypoxia, adenosine), vasoactive neurotransmitters (dopamine, gammaamino butyric acid, acethylcoline), nitric oxide (NO), carbon monoxide (CO), and so on, ${ }^{6,7}$ with a potential contribution from astrocytes. ${ }^{8}$

$\mathrm{CA}$ is one of the homeostatic mechanisms of the body to keep CBF relatively constant despite changes in CPP. Even though the basic mechanisms responsible for neurovascular coupling and autoregulation are yet to be fully understood, it is nevertheless likely that neurovascular coupling and autoregulation share some common pathways that link them. ${ }^{9,10}$ In the next section, we consider CA in more detail.

\section{Cerebral autoregulation: the link between perfusion pressure and cerebral blood flow}

\subsection{Basic mechanisms and physiological importance of cerebral autoregulation}

As discussed above, $\mathrm{CBF}$ is affected by a number of physiological and biochemical mechanisms, including changes in CPP.
$\mathrm{CA}$ is the homeostatic process of regulation of $\mathrm{CBF}$ in response to changes in CPP. The way CA is achieved is through the regulation of CVR, which is done most effectively by modulating the radius of cerebral small arteries and arterioles [see Eq. (1)]. In the absence of CA, an increase in MAP causes an increase of CPP and, therefore, an increase of CBF even if the metabolic demand of the brain remains constant. Therefore, the CA mechanism, which can be seen as a negative feedback loop mechanism, counteracts the MAP increase by narrowing the vessels' radius (thus increasing their resistance to flow) and bringing CBF to the original level. Conversely, a decrease in MAP tends to decrease $\mathrm{CBF}$, and the regulatory mechanism causes vessel dilation to rebalance the CBF. These reactions of the cerebrovascular system to a MAP change occur if CA is working properly, otherwise, in pathological conditions where CA is impaired, CBF follows more or less passively (according to the level of impairment) MAP changes.

The physiological origin of CA is still unclear, with proposed mechanisms invoking myogenic, metabolic, and neurogenic processes. ${ }^{3,11}$ Myogenic mechanism: a myogenic response of vascular smooth muscle to transmural pressure changes was proposed to occur through arterial membrane depolarization, and to result in changes in the concentration of $\mathrm{Ca}^{2+}$ in the arterial wall. ${ }^{12}$ Metabolic mechanism: the altered concentration of vasoactive metabolites (such as adenosine) was proposed to result from initial blood-pressure-induced changes in $\mathrm{BF}^{13}$ Neurogenic mechanism: perivascular neurons were proposed to have autoregulatory effects on cerebral arterioles. ${ }^{14}$ Regardless of which mechanism is responsible or prevalent, CA is mediated through the release of chemical mediators, which implies that a finite amount of time is required to regulate the CVR. Therefore, a finite amount of time is needed to restore the original value of CBF following a MAP change. ${ }^{11}$

\subsection{Static versus dynamic cerebral autoregulation}

Studies on CA can be divided into static and dynamic ones. Even though the mechanisms underlying static and dynamic CA might be the same or share some common basis, the time scale at which they are observed is different: static CA refers to MAP and $\mathrm{CBF}$ values under steady state conditions that are observed over a time scale of minutes or hours, while dynamic CA refers to transient MAP and CBF changes that are observed in a time scale of seconds. Early studies on CA relied on relatively "slow" methods for measuring CBF, like the Kety-Schmidt technique ${ }^{15}$ (see Sec. 3.2.1), or the ${ }^{133} \mathrm{Xe}$ [Ref. 16] or ${ }^{85} \mathrm{Kr}$ [Ref. 17] uptake technique (see Sec. 3.3.1). MAP was changed either by shifting central blood volume with mechanical maneuvers (like changing posture from supine to standing, head-up tilting, or introducing lower-body negative pressure), or, more commonly, by vasoactive drug injection. For a list of methods used to change MAP in static CA studies, we refer to the review by Numan et al. ${ }^{18}$ The measurements of MAP and CBF were taken only at baseline (i.e., before the MAP was changed) and after the effect of the challenging mechanism was complete (usually after minutes). Therefore, with the typical methods used for measuring static $\mathrm{CA}$, it was not possible to study the temporal evolution of the transients in MAP and CBF as they reached their steady-state values. Moreover, according to these methods, CA was conceived as an all-or-nothing mechanism, i.e., either it was present (if $\mathrm{CBF}$ recovered to the initial value) or not (if $\mathrm{CBF}$ followed passively the MAP change). 
With the advent of transcranial Doppler ultrasound (TCD, see Sec. 3.6.1), it was possible to sample the flow velocity of a large cerebral vessel [usually the middle cerebral artery (MCA)] with a high-sampling rate. This capability allowed for new methods of measuring a dynamic CA response. One of the typical MAP challenging mechanisms is the thigh pressure cuff release method, ${ }^{19}$ which will be described in more detail in Sec. 2.2.2. In both static and dynamic CA processes, the regulation of $\mathrm{CBF}$ is confined to the arterial compartment primarily at the level of small arteries and arterioles, which are able to dilate or constrict in order to change their resistance to flow.

\subsubsection{Static cerebral autoregulation}

A first review on static CA was written by Lassen. ${ }^{20}$ The MAPCBF curve reported in this work showed a constant CBF for MAP values between 60 and $170 \mathrm{mmHg}$, indicating a highly active static regulatory system. The work of Lassen had a profound effect on the scientific and medical community, and the MAP-CBF curve presented in his paper was considered an important reference for the upper and lower cutoff values of MAP within which CA was effective. Figure 1 shows the CBF plateau over the MAP range for static autoregulation. The MAP-CBF curve in Lassen's work was obtained by combining the results from seven human studies with 11 different subject groups, where in each group $\mathrm{CBF}$ was measured at a single MAP. The results on different subject groups were mixed regardless of whether the subjects were healthy, diseased, or under medication. Therefore, the curve represented intersubject values under different health conditions, rather than an intrasubject MAP-CBF relationship measured on a cohort of subjects in similar health conditions. As previously noted, this way of extrapolating the CA static curve from a limited number of different subjects can lead to misleading results, even if the subjects are all healthy, because of individual variability and unaccounted for effects of other variables. ${ }^{11}$ It is possible that the static CA curve is more pressure passive than previously described by Lassen, or, in other words, that the CBF-MAP curve in the autoregulation range is not exactly a flat plateau (as in Fig. 1), but has a slightly positive slope. This is in agreement also with some theoretical models of CA based on a feedback loop. ${ }^{21}$ Moreover, it is nowadays known that the static CA curve is affected by other variables, like the concentrations of carbon dioxide $\left(\mathrm{CO}_{2}\right)$ and $\mathrm{O}_{2}$ in blood. For

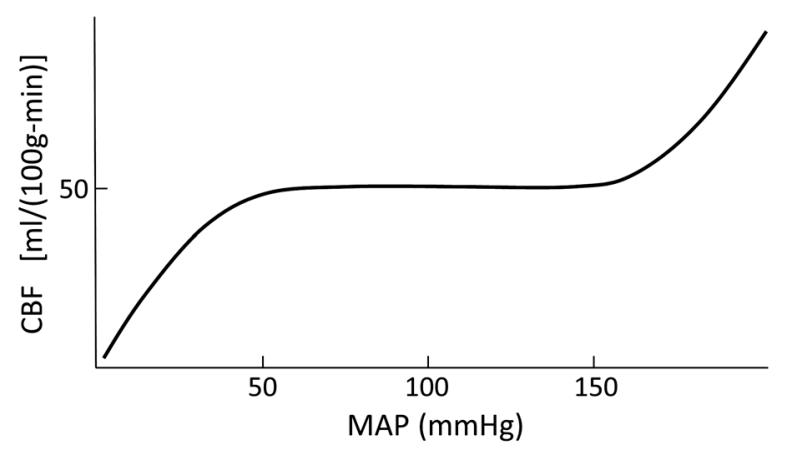

Fig. 1 Static autoregulation. The classic static autoregulation curve shows a plateau of cerebral blood flow (CBF) versus mean arterial pressure (MAP) in the MAP range from about $50 \mathrm{mmHg}$ to about $170 \mathrm{mmHg}$. a theoretical model of the influence of blood gas levels on $\mathrm{CA}$, we refer to the work of Payne et al. ${ }^{22}$

In a more recent review of static $\mathrm{CA},{ }^{18}$ the authors have reanalyzed 49 case studies of healthy subjects, dividing them into two groups according to whether MAP was increased or decreased. Since CBF was measured in different ways in the 49 studies, the authors considered the percentage change of $\mathrm{CBF}$ (relative to baseline) as a function of the percentage change of MAP (relative to baseline). The linear regression coefficient between MAP and CBF was positive and significantly greater during decreases than during increases in MAP, suggesting that autoregulation is more effective against positive changes in MAP than against negative changes in MAP. An asymmetric $\mathrm{CBF}$ response to an increase versus a decrease of MAP was also found in dynamic studies involving patients with TBI. ${ }^{23,24}$

We conclude this section with a comment about studies on static CA. As it is highlighted in the work of Tzeng and Ainslie, ${ }^{3}$ static CA studies pose some difficulties, especially if one wants to reproduce the entire static CA curve, because of confounding effects of some covariates of MAP changes. One of the covariates is the partial pressure of $\mathrm{CO}_{2}$, which can be altered after changes in MAP, and, therefore, it can affect CBF independently from MAP. Also, the use of drugs is necessary for studying large changes in MAP because of the baroreflex mechanism that limits the range of MAP induced by mechanical maneuvers. Therefore, one should be aware of the potential side effects of drugs on the tonus of both large and small arteries, which can also alter the CA response. Since static studies imply relatively long time scales (in the order of minutes or longer) to reach steady state values of MAP and $\mathrm{CBF}$, it can be more difficult to disentangle the pure $\mathrm{CA}$ response to MAP changes from the vascular effects of drugs and from other physiological mechanisms.

\subsubsection{Dynamic cerebral autoregulation}

CA operates at relatively short time scales, as already observed in studies dating back to the 1930s, in which the arteriolar dilation was directly observed on a time scale of seconds following changes in arterial blood pressure (ABP). ${ }^{25}$ In later studies, it was also confirmed that the mechanism of vessel dilation in response to hypotension occurred at a similar temporal scale, as long as the change in MAP was strong enough to elicit a CA response. For example, in some studies, a MAP change of $5 \mathrm{mmHg}$ is used as a threshold for eliciting a CA response. ${ }^{18}$

Aaslid et al. ${ }^{19}$ proposed a method for studying dynamic CA that still remains one of the most commonly used. It is based on the transient change in MAP following a thigh cuff pressure release, after a sustained cuff inflation above the systolic pressure for about 2 to $3 \mathrm{~min}$. The sudden cuff release causes a decrease in MAP of about $20 \mathrm{mmHg}$, which develops over 3 to $5 \mathrm{~s}$ postrelease, before the recovery phase starts. Usually, the entire MAP transient from the moment of cuff release to the recovery to steady state unfolds in a time range of 15 to $20 \mathrm{~s}$ (see Fig. 2). A trend similar to that of MAP is observed in the arterial blood flow velocity $(\mathrm{BFv})$, which is monitored with TCD, usually in the MCA. We observe that, strictly speaking, $\mathrm{BFv}$ is a surrogate of $\mathrm{CBF}$ and it can be considered as a reliable substitute of CBF only if the vessel's diameter does not change. This might be the case in several protocols (including the thigh cuff release protocol), but not in others, as questioned in recent literature. ${ }^{26}$ Also, we note that the BFv and MAP transients usually follow slightly different dynamics, with 


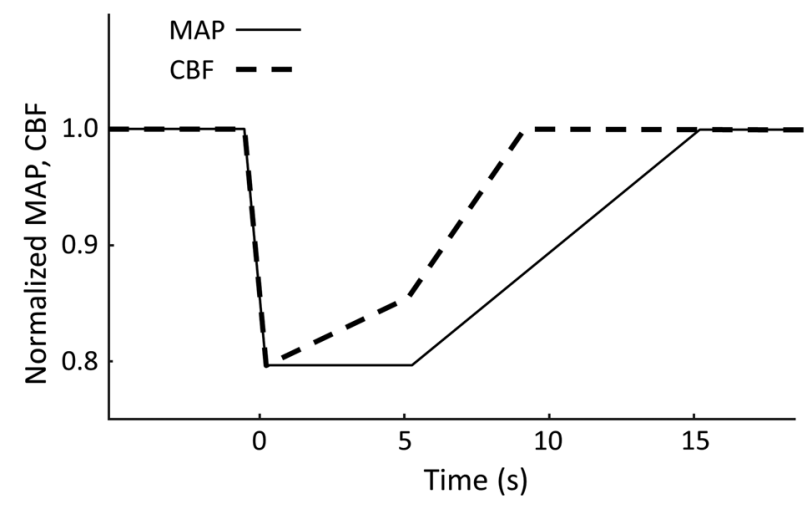

Fig. 2 Dynamic autoregulation. Schematic illustration of the mean arterial pressure (MAP) and cerebral blood flow (CBF) transients induced by the fast release of pneumatic thigh cuffs (at time 0 ) for the assessment of dynamic autoregulation. MAP quickly drops and CBF passively follows this fast change. Then MAP remains at a value lower than at baseline for about $5 \mathrm{~s}$, and during this time autoregulation mechanisms increase CBF toward its precuff-release value. After this initial $\sim 5 \mathrm{~s}$ period, baroreflex mechanisms increase MAP, and CBF changes reflect both MAP changes and autoregulatory effects.

a faster recovery of $\mathrm{BFv}$ to baseline, at least in the case of a properly functioning $\mathrm{CA}$.

Aaslid et al. ${ }^{19}$ have shown that different CBF responses to MAP changes are measured for different partial pressures of $\mathrm{CO}_{2}$ in arterial blood $\left(\mathrm{PaCO}_{2}\right)$. Basically, three cases were studied: hypocapnia (induced by hyperventilation), normocapnia (under normal breathing conditions), and hypercapnia (induced by breathing a mixture of $5 \% \mathrm{CO}_{2}$ in air). The end-expiratory partial pressures of $\mathrm{CO}_{2}\left(\mathrm{pCO}_{2}\right.$, also referred to as end-tidal $\mathrm{CO}_{2}$ ) in the three cases were: $22 \mathrm{mmHg}$ (hypocapnia), $37 \mathrm{mmHg}$ (normocapnia), and $47 \mathrm{mmHg}$ (hypercapnia). The lower the level of $\mathrm{CO}_{2}$, the faster was the recovery of $\mathrm{BFv}$ to baseline after cuff release. This behavior can be expected by considering the known effect of $\mathrm{CO}_{2}$ content in blood on the MAP-CBF curve measured during static CA studies: the curve becomes more parallel to the horizontal MAP axis (indicating better autoregulation) during hypocapnia, whereas it shows a positive slope (i.e., CBF follows more passively MAP changes) during hypercapnia. An intuitive way to explain these results is the following: during hypocapnia the vascular tone is increased with respect to normocapnia (vessels are constricted) and therefore any CA response to hypotensive maneuvers (like during the cuff release) occurs on a faster time scale because it implies a relaxation of the vascular tone (i.e., a dilation of the blood vessels). On the contrary, during hypercapnia, the vascular tone is relaxed with respect to normocapnia (vessels are dilated), and any CA response to hypotensive maneuvers will occur on a slower time scale because it implies a further relaxation of the tonic level (i.e., a further dilatation of the blood vessels). More precisely, the dynamic CA measurements based on the transient changes of MAP and CBF induced by the thigh cuffs release mostly focus on the initial $\sim 5 \mathrm{~s}$ after cuff release, when MAP remains low before baroreflex mechanisms raise it back toward its baseline value. It is the rate of CBF recovery during this initial period, when MAP is approximately constant (at a value lower than at baseline), that is most closely indicative of CA. The main features of MAP and CBF transient changes following thigh cuffs release are schematically illustrated in Fig. 2.
The thigh pressure cuff release method is termed a time domain method, and under the assumption that CA can be approximated to be a linear process (at least for small change of $\mathrm{MAP}^{27}$ ), one can use typical methods of linear systems to analyze the data (see Sec. 5). Other maneuvers to study dynamic CA have been proposed on the basis of induced MAP oscillations; one such method is the lower body negative pressure protocol. ${ }^{28}$ These methods, which are based on inducing oscillations at different frequencies, are termed frequency-domain methods, and can also be studied with typical methods of linear systems (see Sec. 5).

We conclude this section by listing a few advantages of dynamic versus static CA studies. First, the fast MAP-CBF temporal dynamics implied in dynamic CA studies make the confounding effects of some covariates of MAP changes less important than in static CA studies. Second, the study of MAP and CBF transients, like those measured in the thigh cuff release protocol, has elicited a new concept of CA, in which different "levels" of dynamic CA can be distinguished, based on the particular features of the temporal trends of $\mathrm{CBF}$ (see Sec. 5). In other words, CA is viewed as a process characterized by different levels of effectiveness and not as an all-or-nothing mechanism (as it was conceived in previous static CA studies). Third, dynamic studies are easier to perform, do not require invasive maneuvers (like drug injections), and involve shorter acquisition times, facilitating longitudinal studies. One of the major problems of dynamic studies of CA is that they cannot be used to study the vasoconstriction response to hypertension. The reason is that it is difficult to induce hypertension without using drugs that act on a longer temporal scale.

\section{Techniques to measure cerebral blood flow}

In this section, we first provide an overview of some of the general concepts used for measuring $\mathrm{CBF}$, which have been adapted to suit the capabilities of different measurement modalities. We then go into detail for each technique, describing the principle that is used, what needs to be measured, and the units of all terms. Table 1 summarizes key properties of the various techniques for measuring $\mathrm{CBF}$, including their level of invasiveness, spatial extent, ability to provide absolute or relative measurements, and implementation for single reading or continuous monitoring. Table 2 lists relevant acronyms and symbols.

\subsection{General concepts used to measure cerebral blood flow}

\subsubsection{Fick principle}

A general approach to measuring $\mathrm{CBF}$ is based on a mass balance relationship, known as the Fick principle, applied to a physiologically inert substance $x$ (i.e., a substance that is not metabolized in the brain) in the blood stream. In its simplest form, the Fick principle states that the amount of substance $x$ ( $\mathrm{d} Q_{x}$; units: $\operatorname{mol}_{x}$ ) that is accumulated in (or dissipated from) a brain tissue volume $V_{T}$ over the infinitesimal time interval $\mathrm{d} t$ about time $t$ is given by the difference between the amounts of the substance delivered to (by arterial inflow) and removed from (by venous outflow) the tissue volume between times $t$ and $t+\mathrm{d} t$. We observe that the tissue volume $V_{T}$ is intended to include the blood that perfuses it, and that the blood volume fraction, or cerebral blood volume (CBV), is assumed to stay constant. With these definitions and under these conditions, the Fick principle is written as follows: 
Table 1 Main properties of current techniques to measure cerebral blood flow. In this Table, invasive techniques require surgical access, catheterization, or arterial puncture, whereas minimally invasive techniques require intravenous injection of a contrast agent.

\begin{tabular}{|c|c|c|c|c|c|}
\hline Method & Technique & Invasiveness & Spatial extent & $\begin{array}{l}\text { Absolute or } \\
\text { relative }\end{array}$ & $\begin{array}{l}\text { Snapshot or } \\
\text { continuous }\end{array}$ \\
\hline Intravascular & $\mathrm{N}_{2} \mathrm{O}$ inhalation & Invasive & Global & Absolute & Snapshot \\
\hline \multirow[t]{2}{*}{ measurements } & Thermodilution & Invasive & Global & Absolute & Continuous \\
\hline & ${ }^{133} \mathrm{Xe},{ }^{85} \mathrm{Kr}$ & Invasive & Regional & Absolute & Snapshot \\
\hline \multirow{3}{*}{ Nuclear medicine } & SPECT & Minimally invasive & Local & Relative & Snapshot \\
\hline & PET & Minimally invasive & Local & Absolute & Snapshot \\
\hline & $\mathrm{Xe}-\mathrm{CT}$ & Noninvasive & Local & Absolute & Snapshot \\
\hline \multirow[t]{2}{*}{ X-ray imaging } & Perfusion CT & Minimally invasive & Local & Absolute or Relative & Snapshot \\
\hline & DSC-MRI & Minimally invasive & Local & Absolute or Relative & Snapshot \\
\hline \multirow[t]{2}{*}{ Magnetic resonance imaging } & ASL & Noninvasive & Local & Absolute & Snapshot (repeatable) \\
\hline & TCD & Noninvasive & Regional & Relative & Continuous \\
\hline Ultrasound & $\begin{array}{l}\text { Transit-time } \\
\text { ultrasonic flowmetry }\end{array}$ & Invasive & Regional & Relative & Continuous \\
\hline \multirow[t]{3}{*}{ Thermal diffusion } & TDF & Invasive & Regional/local & Absolute & Continuous \\
\hline & LDF & Invasive & Regional/local & Relative & Continuous \\
\hline & DCS & Noninvasive & Regional/local & Relative & Continuous \\
\hline \multirow[t]{3}{*}{ Biomedical optics } & Qualitative NIRS & Noninvasive & Regional/local & Relative & Continuous \\
\hline & Quantitative NIRS & Minimally invasive & Regional/local & Absolute & Snapshot \\
\hline & $\mathrm{CHS}$ & Noninvasive & Regional/local & Absolute & Continuous \\
\hline
\end{tabular}

Note: SPECT: single photon emission computed tomography; PET: positron emission tomography; CT: computed tomography; DSC-MRI: dynamic susceptibility contrast magnetic resonance imaging; ASL: arterial spin labeling; TCD: transcranial Doppler; TDF: thermal diffusion flowmetry; LDF: laser Doppler flowmetry; DCS: diffuse correlation spectroscopy; NIRS: near-infrared spectroscopy; CHS: coherent hemodynamics spectroscopy.

$\mathrm{d} Q_{x}(t)=F_{V_{T}}(t)\left\{[x]_{a}(t)-[x]_{v}(t)\right\} \mathrm{d} t$,

where $F_{V_{T}}$ is the amount of blood that flows through volume $V_{T}$ per unit time (units: $\mathrm{ml}_{\text {blood }} / \mathrm{min}$ ), and $[x]_{a}$ and $[x]_{v}$ are the concentrations of substance $x$ in arterial and venous blood, respectively, [units: $\mathrm{mol}_{x} / \mathrm{ml}_{\text {blood }}$ ]. If we divide both sides of Eq. (2) by the mass $\left(M_{T}\right)$ of the brain tissue in volume $V_{T}$, we obtain

$\mathrm{d} q_{x}(t)=\operatorname{CBF}(t)\left\{[x]_{a}(t)-[x]_{v}(t)\right\} \mathrm{d} t$,

where $\mathrm{d} q_{x}(t)$ is the amount of substance $x$ accumulated in tissue volume $V_{T}$ between times $t$ and $t+\mathrm{d} t$ per unit mass of tissue (units: $\mathrm{mol}_{x} / \mathrm{g}_{\text {tissue }}$ ), and $\mathrm{CBF}=F_{V_{T}} / M_{T}$ is the CBF. If we now consider a finite time interval $\Delta t$ and we further assume that $\mathrm{CBF}$ is constant during this time interval, we can integrate Eq. (3) to obtain:

$\mathrm{CBF}=\frac{\Delta q_{x}}{\int_{0}^{\Delta t}\left\{[x]_{a}(t)-[x]_{v}(t)\right\} \mathrm{d} t}$, where $\Delta q_{x}$ is the amount of substance $x$ accumulated in (if positive) or dissipated from (if negative) the tissue volume $V_{T}$, in the time interval $0-\Delta t$, per unit mass of tissue. Figure 3(a) shows a schematic representation of the Fick principle in the case where $V_{T}$ is the entire brain volume and CBF provides a global measure of brain perfusion.

\subsubsection{Central volume principle}

A case in which a measurement of the tracer concentration in venous blood is not required [unlike the case of Eq. (4)] is when the tracer concentration is directly measured in the brain volume of interest (VOI). If the tracer thoroughly mixes with blood, its concentration in brain tissue $\left(C_{T}\right.$, units: $\mathrm{mol} / \mathrm{g}_{\text {tissue }}$, where, again, we consider brain tissue as a whole, including the vascular space) at a given time $t$ is given by the integration of its concentration in arterial blood $\left(C_{a}\right.$, units: $\left.\mathrm{mol} / \mathrm{ml}_{\mathrm{blood}}\right)$ at a previous time $\tau$ (ranging from $-\infty$ to $t$ ) times the probability that the tracer transit time in the VOI is longer than $t-\tau[R(t-\tau)$, dimensionless]: 
Fantini et al.: Cerebral blood flow and autoregulation: current measurement techniques and prospects for noninvasive optical methods

Table 2 Acronyms/symbols for quantities and variables reported in this work with associated definition and units.

\begin{tabular}{|c|c|c|}
\hline Acronym/Symbol & Definition & Units \\
\hline $\mathrm{BFI}$ & Blood flow index & $\mathrm{cm}^{2} / \mathrm{s}$ \\
\hline BFv & Blood flow velocity & $\mathrm{cm} / \mathrm{s}$ \\
\hline $\mathrm{CA}$ & Cerebral autoregulation & $\mathrm{N} / \mathrm{A}$ \\
\hline $\mathrm{cbf}$ & Cerebral blood flow (relative changes) & - \\
\hline CBF & Cerebral blood flow & $\mathrm{ml}_{\text {blood }} /\left(100 \mathrm{~g}_{\text {tissue }} \min \right)$ \\
\hline CBFv & Cerebral blood flow velocity & $\mathrm{cm} / \mathrm{s}$ \\
\hline CBV & Cerebral blood volume & $\mathrm{ml}_{\text {blood }} / 100 \mathrm{~g}_{\text {tissue }}$ \\
\hline CPP & Cerebral perfusion pressure & $\mathrm{mmHg}$ \\
\hline CVR & Cerebrovascular resistance & $\mathrm{mmHg} /\left[\mathrm{ml}_{\text {blood }} /\left(100 \mathrm{~g}_{\text {tissue }} \min \right)\right]$ \\
\hline ICP & Intracranial pressure & $\mathrm{mmHg}$ \\
\hline JBF & Jugular blood flow & $\mathrm{ml}_{\text {blood }} / \mathrm{min}$ \\
\hline MAP & Mean arterial pressure & $\mathrm{mmHg}$ \\
\hline $\mathrm{MTT}_{B}$ & Mean transit time of blood or nondiffusible tracer & $\mathrm{s}$ \\
\hline $\mathrm{MTT}_{D}$ & Mean transit time of diffusible tracer & $\mathrm{s}$ \\
\hline VBF & Volumetric blood flow & $\mathrm{ml} / \mathrm{s}$ \\
\hline$A$ & Area under tracer's clearance curve & $\mathrm{ml}_{\text {tracer }} / \mathrm{ml}_{\text {tissue }} \times \min$ \\
\hline$c_{B}, c_{l}$ & Specific heat of blood, indicator & $\mathrm{cal} /\left(\mathrm{g}^{\circ} \mathrm{C}\right)$ \\
\hline$c_{s}$ & Speed of ultrasound in tissue & $\mathrm{m} / \mathrm{s}$ \\
\hline$C_{a}$ & Concentration of tracer in arterial blood & $\mathrm{mol} / \mathrm{ml}_{\text {blood }}$ \\
\hline$C_{T}$ & Concentration of tracer in brain tissue & $\mathrm{mol} / \mathrm{g}_{\text {tissue }}$ \\
\hline $\mathrm{ctHb}$ & Concentration of hemoglobin in blood & $\mathrm{mol}_{\mathrm{HbT}} / \mathrm{ml}_{\text {blood }}$ \\
\hline$D_{B}$ & Effective Brownian diffusion coefficient & $\mathrm{cm}^{2} / \mathrm{s}$ \\
\hline $\mathscr{F}^{(c)}$ & Fåhraeus factor & - \\
\hline$f$ & Frequency & $\mathrm{Hz}$ \\
\hline$F_{1}$ & Injection rate of indicator fluid & $\mathrm{ml} / \mathrm{min}$ \\
\hline$F_{V_{T}}$ & Amount of blood flowing through tissue volume $V_{T}$ per unit time & $\mathrm{ml}_{\text {blood }} / \min$ \\
\hline$h(t)$ & Probability density of tracer transit time & - \\
\hline$H$ & Maximum tracer concentration & $\mathrm{ml}_{\text {tracer }} / \mathrm{ml}_{\text {tissue }}$ \\
\hline$[\mathrm{Hb}], D$ & Tissue concentration of deoxyhemoglobin & $\mathrm{mol}_{\mathrm{Hb}} / \mathrm{ml}_{\text {tissue }}$ \\
\hline$[\mathrm{HbD}]$ & Difference $\left[\mathrm{HbO}_{2}\right]-[\mathrm{Hb}]$ & $\mathrm{mol} / \mathrm{ml}_{\text {tissue }}$ \\
\hline$\left[\mathrm{HbO}_{2}\right], O$ & Tissue concentration of oxyhemoglobin & $\mathrm{mol}_{\mathrm{HbO} 2} / \mathrm{ml}_{\text {tissue }}$ \\
\hline$[\mathrm{HbT}], T$ & Tissue concentration of total hemoglobin & $\mathrm{mol}_{\mathrm{HbT}} / \mathrm{ml}_{\text {tissue }}$ \\
\hline$L$ & Length of vascular segment & $\mathrm{mm}$ \\
\hline$M_{T}$ & Brain tissue mass & g \\
\hline
\end{tabular}


Table 2 (Continued).

\begin{tabular}{|c|c|c|}
\hline Acronym/Symbol & Definition & Units \\
\hline$\overline{M_{T}^{0}}$ & Tissue magnetization before inversion pulse & $\mathrm{J} /\left(\mathrm{T}-\mathrm{ml}_{\text {tissue }}\right)$ \\
\hline$M_{T}^{s s}$ & Tissue magnetization at steady state & $\mathrm{J} /\left(\mathrm{T}-\mathrm{ml}_{\text {tissue }}\right)$ \\
\hline$\delta P$ & Pressure difference for a vascular segment & $\mathrm{mmHg}$ \\
\hline $\mathrm{d} q_{x}\left(\Delta q_{x}\right)$ & $\begin{array}{l}\text { Amount of tracer } x \text { accumulated in tissue volume } V_{T} \\
\text { over time } \mathrm{d} t(\Delta t) \text { per unit mass }\end{array}$ & $\mathrm{mol}_{x} / \mathrm{g}_{\mathrm{tissue}}$ \\
\hline $\mathrm{d} Q_{x}$ & Amount of tracer $x$ accumulated in tissue volume $V_{T}$ over time $\mathrm{d} t$ & $\operatorname{mol}_{x}$ \\
\hline$r$ & Radius of blood vessel & $\mathrm{mm}$ \\
\hline$R(t)$ & Impulse residue function & - \\
\hline $\mathrm{SaO}_{2}, S^{(a)}$ & Arterial hemoglobin saturation & - \\
\hline $\mathrm{ScO}_{2}, \mathrm{StO}_{2}$ & Cerebral oxygen saturation of hemoglobin & - \\
\hline $\mathrm{SvO}_{2}, S^{(v)}$ & Venous hemoglobin saturation & - \\
\hline$t^{(c)}$ & Capillary transit time & s \\
\hline$T_{B}, T_{l}, T_{M}$ & Temperature of blood, indicator, mixture & ${ }^{\circ} \mathrm{C}$ \\
\hline$T_{1}$ app & Time constant of decay from $M_{T}^{0}$ to $M_{T}^{s s}$ & s \\
\hline$v_{B}$ & Speed of red blood cells & $\mathrm{cm} / \mathrm{s}$ \\
\hline$V_{T}$ & Selected volume of brain tissue & $\mathrm{mm}^{3}$ \\
\hline$[x]_{a}$ & Concentration of tracer $x$ in arterial blood & $\mathrm{mol}_{x} / \mathrm{ml}_{\text {blood }}$ \\
\hline$[x]_{v}$ & Concentration of tracer $x$ in venous blood & $\mathrm{mol}_{x} / \mathrm{ml}_{\text {blood }}$ \\
\hline$\alpha$ & Ratio of moving to total scatterers & - \\
\hline$\eta$ & Blood viscosity & $\mathrm{cP}$ \\
\hline$\lambda$ & Tissue-blood partition coefficient & $\mathrm{ml}_{\text {blood }} / \mathrm{g}_{\text {tissue }}$ \\
\hline$\rho_{B}, \rho_{l}$ & Mass density of blood, indicator & $\mathrm{g} / \mathrm{ml}$ \\
\hline
\end{tabular}

$C_{T}(t)=\mathrm{CBF} \int_{-\infty}^{t} C_{a}(\tau) R(t-\tau) \mathrm{d} \tau$.

$R(t)$ is also referred to as the impulse residue function, and it represents the fraction of the tracer input at time 0 that is still present in the VOI at time $t$. The integral in Eq. (5) is a temporal convolution between the arterial concentration of the tracer and the impulse residue function. The factor $\mathrm{CBF}$ on the right hand side accounts for the tracer flow through the blood stream and translates the $C_{a}$ units of concentration per unit volume of blood into the $C_{T}$ units of concentration per unit mass of tissue. A representative time trace $C_{T}(t)$ for a tracer bolus is shown in Fig. 3(b).

Now, let us introduce the probability density function, $h(t)$, for the tracer transit time within the tissue VOI $\left(V_{T}\right)$. This probability density function leads to the definition of the tracer mean transit time (MTT) within such tissue region of interest

$\operatorname{MTT}=\int_{0}^{\infty} t h(t) \mathrm{d} t$.
We also observe that the probability of a residence transit time longer than $t$, introduced in Eq. (5) as $R(t)$ is

$R(t)=\int_{t}^{\infty} h(\tau) \mathrm{d} \tau=1-\int_{0}^{t} h(\tau) \mathrm{d} \tau$.

Nondiffusible tracer: In the case of a nondiffusible tracer, i.e., a tracer that remains confined to the vascular space, its MTT transit [given by Eq. (6)] coincides with the blood transit time, and, therefore, we denote it with a subscript " $B$ " $\left(\mathrm{MTT}_{B}\right)$. Now, let us consider the blood volume that is contained within the tissue VOI, $V_{T}$, and let us denote it with $V_{B}$ (this blood volume has units of $\mathrm{ml}$, and should not be confused with $\mathrm{CBV}$, which has units of $\mathrm{ml}_{\text {blood }} / \mathrm{ml}_{\text {tissue }}$ or $\mathrm{ml}_{\text {blood }} / 100 \mathrm{~g}_{\text {tissue }}$ and actually represents a blood volume fraction). Such blood volume $V_{B}$ can be decomposed into infinitesimal elements $\left[\mathrm{d} V_{B}(t)\right]$ that are associated with a given blood transit time $t$ or, more accurately, with a transit time within the interval $(t, t+\mathrm{d} t)$. This infinitesimal element $\mathrm{d} V_{B}(t)$ is given by the product of two factors: (1) the rate at which blood volume elements with transit times between $t$ and $t+\mathrm{d} t$ enter the tissue 
Fick principle
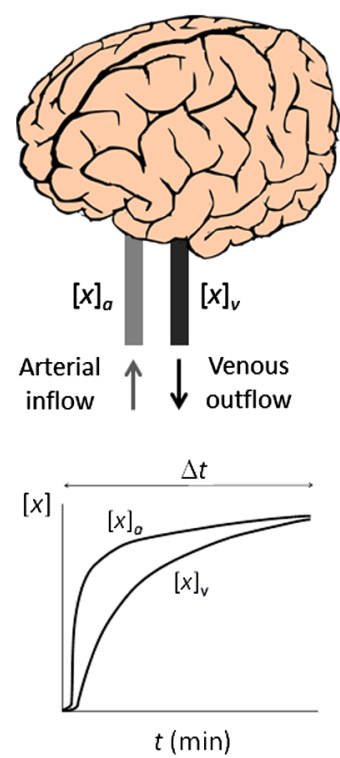

(a)
Central volume principle
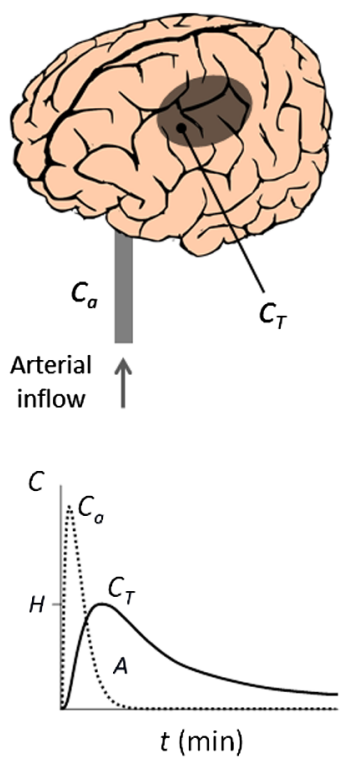

(b)
Doppler, autocorrelation
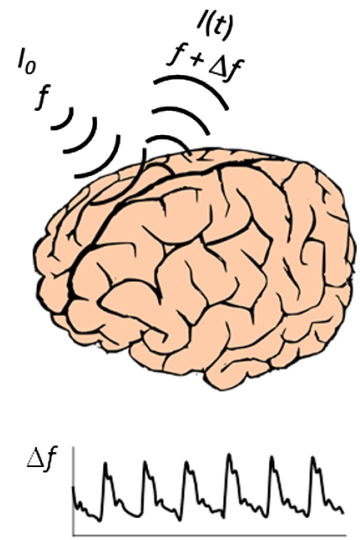

$t(\mathrm{~s})$

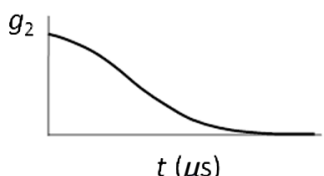

(c)

Fig. 3 Three basic approaches to the measurement of cerebral blood flow. (a) The Fick principle, (b) the central volume principle, and (c) the Doppler effect or autocorrelation methods. (a) A global CBF measurement is based on recording time traces of the arterial and venous blood concentrations $\left([\boldsymbol{X}]_{a}\right.$ and $[\boldsymbol{X}]_{v}$, respectively) of a diffusible and physiologically inert intravascular tracer $x$ over a time $\Delta t$ that is sufficiently long to achieve equilibrium in the blood-brain tracer diffusion (in the case of the KetySchmidt method, the intravascular tracer was nitrous oxide $\left(\mathrm{N}_{2} \mathrm{O}\right)$, breathed continuously by the subject [see Sec. 3.2.1]). (b) A regional CBF measurement in a volume of interest $V_{T}$ is based on the measurement of the temporal dynamics of the arterial and tissue concentration $\left(C_{a}\right.$ and $C_{T}$, respectively) of an intravascular bolus $(H$ and $A$ represent the peak value and the total area under the curve of the temporal trace of $C_{T}$ (this approach is the basis for a number of nuclear medicine [Sec. 3.3], X-ray [Sec. 3.4], and MRI techniques [Sec. 3.5]). (c) The Doppler effect applies to ultrasound or optical waves that interrogate the brain tissue at a certain frequency $f$, and results in a frequency shift $(\Delta f)$ and in a decay rate for the normalized intensity autocorrelation function $\left(g_{2}\right)$ that are directly related to the speed of blood flow (these methods are employed by Doppler ultrasound [Sec. 3.6.1], laser Doppler flowmetry [Sec. 3.8.1], and diffuse correlation spectroscopy [Sec. 3.8.2]).

region of interest (which is the same as the rate at which they exit it because of the assumed stationarity of CBF and CBV), which is given by $F_{V_{T}} h(t) \mathrm{d} t$ (where $F_{V_{T}}$ represents the amount of blood flowing through the tissue volume $V_{T}$, and has units of $\mathrm{ml}_{\text {blood }} / \mathrm{min}$ ); (2) the time spent by such blood volume elements in the tissue VOI, which is $t$ by definition. Therefore, one can write 29,30

$\mathrm{d} V_{B}(t)=F_{V_{T}} t h(t) \mathrm{d} t$.

Integration of Eq. (8) over all transit times $(0 \leq t<\infty)$ yields the relationship between $F_{V_{T}}$ and $V_{B}$ that is a manifestation of the central volume principle

$F_{V_{T}}=\frac{V_{B}}{\mathrm{MTT}_{B}}$

Dividing both sides of Eq. (9) by the mass $\left(M_{T}\right)$ of the tissue region of interest, and considering that $\mathrm{CBF}=F_{V_{T}} / M_{T}$ and $\mathrm{CBV}=V_{B} / M_{T}$, we can write the central volume principle relationship for $\mathrm{CBF}$ and $\mathrm{CBV}$

$\mathrm{CBF}=\frac{\mathrm{CBV}}{\mathrm{MTT}_{B}}$
Diffusible tracer: Equation (10) may be generalized to the case of a diffusible tracer. In this case, $V_{B}$ in Eq. (9) is replaced by the equilibrium volume of distribution of the tracer, i.e., the blood volume that contains the same amount of tracer that is in the tissue volume $V_{T}$ at equilibrium. ${ }^{31}$ We stress again that "tissue" is intended here to include the blood in the vasculature. ${ }^{32}$ The numerator on the right-hand-side of Eq. (10) then becomes the volume of distribution of the tracer per unit mass of tissue, which is referred to as the tissue-blood partition coefficient, $\lambda$, also defined as the concentration of the diffusible tracer in tissue (at equilibrium) divided by the concentration of the tracer in blood. If the same units for the concentrations in tissue and blood are used, $\lambda$ is dimensionless (or expressed in $\mathrm{ml}_{\text {blood }} / \mathrm{ml}_{\text {tissue }}$ or $g_{\text {blood }} / g_{\text {tissue }}$ ), whereas it takes units of $\mathrm{ml} / \mathrm{g}$ (i.e., $\mathrm{ml}_{\text {blood }} / g_{\text {tissue }}$ ) if the tissue and blood concentrations of the indicator are expressed per unit tissue mass and per unit blood volume, respectively. Both conventions are used in the literature, but here we use the latter convention, so that the units of $\lambda$ and $\mathrm{CBV}$ are the same. For example, the brainblood partition coefficient for water is $0.90 \mathrm{ml} / \mathrm{g},{ }^{33}$ which can be interpreted by saying that $0.90 \mathrm{ml}$ of blood contain the same amount of water as $1 \mathrm{~g}$ of brain tissue. On the basis of the above definitions, the central volume principle equation for a diffusible tracer is 


$$
\mathrm{CBF}=\frac{\lambda}{\mathrm{MTT}_{D}},
$$

where $\mathrm{MTT}_{D}$ is the MTT transit of the diffusible tracer in the tissue VOI (which in general is different from $\mathrm{MTT}_{B}$ ). We conclude this section by observing that $\lambda \sim 1 \mathrm{ml} / \mathrm{g}$ for freely diffusible tracers, whereas for nondiffusible tracers $\lambda=\mathrm{CBV}$, which is about $0.04 \mathrm{ml} / \mathrm{g}$. Therefore, a comparison of Eqs. (10) and (11) shows that $\mathrm{MTT}_{D} \gg \mathrm{MTT}_{B}$, and in fact $\mathrm{MTT}_{D}$ is on the order of minutes whereas $\mathrm{MTT}_{B}$ is on the order of seconds. These concepts are illustrated in Fig. 3(b), in which the indicated time scale of minutes for the dynamics of $C_{T}(t)$ refers to a diffusible tracer.

\subsubsection{Doppler effect and intensity fluctuations}

The interaction of a probing wave, be it an ultrasound pressure wave or an optical electromagnetic wave, with moving red blood cells in the blood stream results in a frequency shift and in intensity fluctuations of the detected wave. The frequency shift is a manifestation of the Doppler effect; the intensity fluctuations can be characterized by the field or intensity autocorrelation functions and their rate of decay. The Doppler effect, which results in spectral line broadening, and the intensity fluctuations are different aspects of the same phenomenon. In fact, the intensity power spectrum and the field autocorrelation function are related by a Fourier transformation, known as the WienerKhinchin theorem. BF velocity, and therefore CBF, is directly related to the Doppler shift $(\Delta f)$ and directly related to the rate of decay of the normalized intensity autocorrelation function $\left(g_{2}\right)$. This approach to the measurement of BF is schematically illustrated in Fig. 3(c). It is important to observe that Doppler and autocorrelation methods do not really measure CBF, but they rather yield measures of the speed of BF, either in large vessels (Doppler ultrasound, Sec. 3.6.1) or in the microcirculation [laser Doppler flowmetry (LDF), Sec. 3.8.1; diffuse correlation spectroscopy (DCS), Sec. 3.8.2]. Because CBF is intended to represent the rate of inflow of arterial blood into the capillary bed rather than the speed of BF within brain tissue, the data provided by Doppler and autocorrelation methods need to be carefully interpreted and are often taken to provide relative measures of $\mathrm{CBF}$ changes.

\subsection{Direct measurements of intravascular tracers in the blood stream}

\subsubsection{Kety-Schmidt arteriovenous difference method}

A seminal paper by Kety and Schmidt, ${ }^{34}$ which set the stage for the development of a variety of $\mathrm{CBF}$ measurements in the human brain, ${ }^{35}$ considered nitrous oxide $\left(\mathrm{N}_{2} \mathrm{O}\right)$ as a freely diffusible intravascular tracer. It was administered by inhalation, and the dynamics of $\left[\mathrm{N}_{2} \mathrm{O}\right]_{a}$ and $\left[\mathrm{N}_{2} \mathrm{O}\right]_{v}$ were measured in blood drawn by femoral artery puncture and from a needle in the right internal jugular vein, respectively. The dynamic measurements were performed over a time interval $\Delta t=10 \mathrm{~min}$ following the beginning of $\mathrm{N}_{2} \mathrm{O}$ inhalation, at which time a steady state was reached, such that $\left[\mathrm{N}_{2} \mathrm{O}\right]_{a}=\left[\mathrm{N}_{2} \mathrm{O}\right]_{v}$. This steady state carries no information about $\mathrm{CBF}$, but the time required to reach it is inversely related to CBF. Specifically, the measurement of $\mathrm{CBF}$ is based on the amount of $\mathrm{N}_{2} \mathrm{O}$ delivered to the brain, per unit brain mass, over the entire time $\Delta t$ $\left(\Delta q_{x}\right)$, and the total arteriovenous difference integrated over time $\Delta t$, as given by Eq. (4), written again here with the generic tracer $x$ replaced by $\mathrm{N}_{2} \mathrm{O}: 34,36$

$$
\mathrm{CBF}=\frac{\Delta q_{\mathrm{N}_{2} \mathrm{O}}}{\int_{0}^{\Delta t}\left(\left[\mathrm{~N}_{2} \mathrm{O}\right]_{a}-\left[\mathrm{N}_{2} \mathrm{O}\right]_{v}\right)(t) \mathrm{d} t} .
$$

Typical time traces of $\left[\mathrm{N}_{2} \mathrm{O}\right]_{a}(t)$ and $\left[\mathrm{N}_{2} \mathrm{O}\right]_{v}(t)$ are shown in Fig. 3(a). The total amount of $\mathrm{N}_{2} \mathrm{O}$ delivered to the brain per unit mass [i.e., the numerator of Eq. (12)] was calculated on the basis of the relative solubility of $\mathrm{N}_{2} \mathrm{O}$ in brain tissue and in blood at equilibrium. Measurements with this method reflect a global CBF, and Kety and Schmidt found a value of $62 \pm 12 \mathrm{ml} /(100 \mathrm{~g} \mathrm{~min})$ in a group of 11 human subjects. ${ }^{34}$

Following its introduction in 1945, the Kety-Schmidt method quickly became a standard approach for quantitative measurements of the global CBF. However, its application is invasive and cumbersome, requiring inhalation of nitrous oxide, puncture or catheterization of the carotid or femoral artery, and accurate measurements of $\mathrm{N}_{2} \mathrm{O}$ concentrations in blood samples. Furthermore, it relies on some assumptions (jugular venous blood representing brain venous drainage, reaching a steady state of brain saturation with $\mathrm{N}_{2} \mathrm{O}$ within 10 to $15 \mathrm{~min}$, and so on) that may not always be accurate. Finally, the KetySchmidt method allows for only global CBF measurements, but regional CBF measurements are highly desirable in a number of research and clinical situations. For these reasons, other techniques have been considered to improve upon the intravascular tracer approach of Kety and Schmidt. For example, the introduction of radioactive tracers (such as ${ }^{133} \mathrm{Xe}$ and ${ }^{85} \mathrm{Kr}$ ), in conjunction with measurements of their clearance curve (i.e., their concentration decay versus time), leads to measurements of regional CBF (see Sec. 3.3.1).

\subsubsection{Jugular thermodilution}

As an alternative to intravascular tracers that diffuse into the brain, one may inject a cold fluid miscible with blood (typically saline) that introduces a thermal perturbation to perform a direct measurement of BF velocity within a large blood vessel that drains the brain, such as the internal jugular vein. This method allows for continuous or repeated measurements (in the cases of continuous infusion or cold bolus input, respectively) and is referred to as jugular thermodilution. ${ }^{37}$ While this method does not require arterial puncture or catheterization (but it requires jugular vein catheterization) it provides only a local measurement of jugular venous flow (in units of $\mathrm{ml}_{\text {blood }} / \mathrm{min}$ ). In the case of a continuous infusion, the jugular blood flow (JBF: $\mathrm{ml} / \mathrm{min}$ ) is expressed as follows in terms of the injection rate of the indicator fluid $\left(F_{I}: \mathrm{ml} / \mathrm{min}\right)$, the specific heat of blood and indicator $\left[c_{B}, c_{I}: \mathrm{cal} /\left(g^{\circ} \mathrm{C}\right)\right]$, and the densities of blood and indicator $\left(\rho_{B}, \rho_{I}: \mathrm{g} / \mathrm{ml}\right)$

$$
\mathrm{JBF}=F_{I} \frac{\rho_{I} c_{I}}{\rho_{B} c_{B}}\left(\frac{T_{M}-T_{I}}{T_{B}-T_{M}}\right),
$$

where $T_{B}, T_{I}$, and $T_{M}$ are the temperatures of blood, the indicator, and their mixture, respectively, which are measured by thermistors placed inside and outside the catheter used to inject the indicator fluid. ${ }^{38}$ The JBF may be translated into a measure of the $\mathrm{CBF}$, by considering that there are two internal jugular veins that drain the entire brain tissue mass $\left(M_{T}: \mathrm{g}\right)$ 


$$
\mathrm{CBF}=\frac{2 \mathrm{JBF}}{M_{T}} .
$$

Measurements of CBF with jugular thermodilution were validated by comparison with measurements with the KetySchmidt method. ${ }^{38}$

While jugular thermodilution allows for continuous measurements of $\mathrm{BF}$, it does require injection of the fluid indicator, so that the ability of the patient to handle the fluid load poses a limitation to the duration of flow monitoring.

\subsection{Nuclear medicine}

\subsubsection{Intra-arterial injection of a radioactive inert gas} $\left({ }^{133} \mathrm{Xe}\right.$ or $\left.{ }^{85} \mathrm{Kr}\right)$

A common intraoperative method to measure CBF is based on the intra-arterial injection of a radioactive bolus that emits $\gamma$-rays (photons) or $\beta$ particles (electrons) and the detection of the diffusible tracer's clearance curve in brain tissue regions by scintillation detectors, ${ }^{31,39}$ an Anger camera, ${ }^{40}$ or a Geiger-Müller tube. ${ }^{41,42}$ Two radioactive isotopes, ${ }^{133} \mathrm{Xe}$ and ${ }^{85} \mathrm{Kr}$, the former more commonly used than the latter, decay by emitting $\gamma$-rays and $\beta$ particles, respectively, that are detected to yield regional radioisotopes clearance curves. This approach relies on the same concepts as the Kety-Schmidt method (Sec. 3.2.1), but allows for regional measurements of $\mathrm{CBF}$, and measures the clearance curve of the tracer continuously rather than at a number of time points. Three methods have been proposed to analyze the clearance curve to generate $\mathrm{CBF}$ measurements:

1. Stochastic analysis is based on the central volume principle [Eq. (11)] and yields the following expression for $\mathrm{CBF}:{ }^{31,43}$

$$
\mathrm{CBF}=\frac{\lambda}{\mathrm{MTT}_{D}}=\lambda \frac{H}{A},
$$

where $\lambda$ is the tissue-blood partition coefficient for the tracer (i.e., the equilibrium volume of distribution of the tracer in blood per gram of tissue) (units: $\left.\mathrm{ml}_{\text {blood }} / g_{\text {tissue }}\right), H$ is the maximum tracer concentration in the region of interest (i.e., the maximum value of the tracer's clearance curve; units: $\mathrm{ml}_{\text {tracer }} / \mathrm{ml}_{\text {tissue }}$ ), $A$ is the area under the tracer's clearance curve (units: $\mathrm{ml}_{\text {tracer }} / \mathrm{ml}_{\text {tissue }} \times \min$ ), and $\mathrm{MTT}_{D}$ is the tracer MTT mean through the tissue region of interest. $H$ and $A$ are visually defined in Fig. 3(b). We recall that the tissue-blood partition coefficient expresses the ratio between the tracer concentration in tissue and in blood, and its value is typically assumed, possibly including a dependence on temperature and hematocrit. ${ }^{44}$ Equation (15) allows for the measurement of CBF in a region of interest from measurements of the clearance curve and knowledge of the tissue-blood partition coefficient of a radioactive tracer.

2. Compartmental analysis considers two tissue compartments (gray and white matter) and assumes diffusion equilibrium of the radioactive tracer in both compartments, where the tracer's concentration in tissue $\left(C_{T_{-} i} ; i=1,2\right)$ is represented by a single exponential function $^{45}$
$C_{T_{-} i}(t)=C_{T_{-} i}(0) \mathrm{e}^{-\frac{\mathrm{CBF}_{i}}{\lambda_{i}} t}$,

where $\lambda_{i}$ is the tissue-blood partition coefficient in gray matter $(i=1)$ or white matter $(i=2)$, and $C_{T_{-} i}(0)$ is the peak value of the tracer's concentration at time 0 . Under the assumption that the bolus arrives rapidly to the tissue investigated, $C_{T_{-} i}(0)$ is proportional to $\mathrm{CBF}_{i} \cdot{ }^{31}$ Moreover, if the two partition coefficients are known, the two values of $\mathrm{CBF}$ in white and gray matter can be calculated from the clearance curve. ${ }^{31}$ It is important to observe that the time constant of the exponential decay in Eq. (16) is $\mathrm{MTT}_{D_{-} i}$, the tracer's transit time in the $i$ 'th tissue compartment [see Eq. (15)].

3. Initial slope analysis considers a single brain tissue compartment and the initial decay (in practice, the first minute or so of the tracer's clearance) of the exponential function of Eq. (16), which is illustrated in Fig. 3(b). From Eq. (16), after removal of the tissue compartment index $i$ and taking the natural $\log$ of both sides of the equation, it immediately follows that: ${ }^{46}$

$$
\mathrm{CBF}=-\lambda \frac{\mathrm{d}}{\mathrm{d} t}\left\{\ln \left[C_{T}(t)\right]\right\} .
$$

We stress that the ${ }^{133} \mathrm{Xe}$ and ${ }^{85} \mathrm{Kr}$ methods rely on the measurement of the clearance of the radioactive tracer from the tissue, a clearance that is assumed to follow an exponential decay expressed as $\exp [-(\mathrm{CBF} / \lambda) t]$. This means that the relevant measurements are conducted after the peak value of $C_{T}(t)$ (indicated as $H$ in Fig. 3(b)) over a time scale of minutes, which is much longer than the time scale of $\sim 10 \mathrm{~s}$ for the arterial bolus $C_{a}(t)$. This approach contrasts with positron emission tomography (PET) methods, which measure the delivery rather than the clearance of the tracer (see Sec. 3.3.3).

\subsubsection{Single-photon emission computed tomography}

Single-photon emission computed tomography (SPECT) uses the injection of a delivery compound, such as hexamethylpropyleneamine oxime (HMPAO) or ethyl cysteinate dimer (ECD), labeled with the radioisotope technetium-99m $\left({ }^{99 \mathrm{~m}} \mathrm{Tc}\right)$, the most common of the gamma ray producing radionuclides. ${ }^{47,48}$ The compound is injected intravenously, passes the blood brain barrier, and is metabolized and retained intracellularly. ${ }^{49,50}$ The SPECT system measures the spatial distribution of the radiotracer in the cerebral tissue and its temporal evolution. Rather than absolute CBF, SPECT measurements yield brain perfusion indices ${ }^{51,52}$ that reflect $\mathrm{CBF}$ as well as the radiotracer's kinetics.

\subsubsection{Positron emission tomography}

CBF imaging with PET, which achieves a spatial resolution on the order of $1 \mathrm{~cm}^{3}$, uses ${ }^{15} \mathrm{O}$-labeled oxygen $\left({ }^{15} \mathrm{O}_{2}\right)$, carbon dioxide $\left(\mathrm{C}^{15} \mathrm{O}_{2}\right)$, or water $\left(\mathrm{H}_{2}{ }^{15} \mathrm{O}\right)$ as a radioactive diffusible contrast agent. After intravenous injection or inhalation of 
the contrast agent, the local instantaneous tissue radiotracer concentration (expressed as $c_{T}^{\left({ }^{15} \mathrm{O}\right)}(t)$, in units of $\mathrm{cps} / \mathrm{g}$, where cps are counts-per-second) is written as follows by the autoradiographic method, developed from the one-compartment Kety-Schmidt method: ${ }^{53,54}$

$$
\left.c_{T}^{\left({ }^{15} \mathrm{O}\right)}(t)=\mathrm{CBF} \int_{0}^{t} c_{a}^{(15} \mathrm{O}\right)(\tau) e^{-\frac{\mathrm{CBF}}{\lambda}(t-\tau)} \mathrm{d} \tau,
$$

where $\left.c_{a}^{(15} \mathrm{O}\right)(t)$ is the arterial concentration of the radiotracer (expressed in cps/ml) and $\lambda$ is the tissue-blood equilibrium partition coefficient for the radiotracer. Equation (18) is formally identical to Eq. (5), with $R(t-\tau)$ replaced by the exponentially decaying factor $\exp (-\mathrm{CBF} / \lambda(t-\tau))$, and the integral is carried out for times that follow the introduction of the radioactive agent at $t=0$. This exponentially decaying factor describes the impulse residue function $R(t-\tau)$ in conjunction with the approximation that the time evolution of the tracer concentration (the tracer dilution curve) is described by a gamma function. ${ }^{55}$ The factor $\mathrm{CBF} / \lambda$ in the exponent of the residence probability function is the inverse of the tracer mean transit time $\left(\mathrm{MTT}_{D}\right)$, in agreement with the central volume principle of Eq. (11). While $c_{a}^{\left({ }^{15} \mathrm{O}\right)(t)}$ can be measured by repeated arterial blood sampling, and $\lambda$ may be estimated, $c_{T}^{\left({ }^{15} \mathrm{O}\right)(t)}$ cannot be measured directly with PET, which measures the total (rather than the instantaneous) number of counts, per unit mass of tissue, over the duration of the PET scan. If the scan occurs between times $t_{1}$ and $t_{2}$, the measured number of counts per unit tissue mass is $\left.\left.C_{T}^{(15} \mathrm{O}\right)=\int_{t_{1}}^{t_{2}} c_{T}^{(15} \mathrm{O}\right)(t) \mathrm{d} t$. Therefore, $\mathrm{CBF}$ is obtained by numerically solving the following equation ${ }^{53}$

$$
\left.C_{T}^{(15 \mathrm{O})}=\mathrm{CBF} \int_{t_{1}}^{t_{2}}\left[\int_{0}^{t} c_{a}^{(15} \mathrm{O}\right)(\tau) \mathrm{e}^{-\frac{\mathrm{CBF}}{\lambda}(t-\tau)} \mathrm{d} \tau\right] \mathrm{d} t^{\prime} .
$$

The measurement time interval $\left(t_{1}, t_{2}\right)$ typically covers the first $40 \mathrm{~s}$ following the bolus arrival, thus mostly relying on the dynamics of tracer delivery rather than tracer clearance (which instead is measured in the ${ }^{133} \mathrm{Xe}$ and ${ }^{85} \mathrm{Kr}$ methods). In general, the dynamics of tracer delivery provides a more reliable measure of CBF because it is mostly determined by the arterial inflow, whereas the dynamics of tracer clearance is also affected by diffusion effects, multiple tissue compartments, and so on.

In addition to $\mathrm{CBF}$, PET can measure $\mathrm{CBV}$, cerebral metabolic rate of oxygen or glucose, and oxygen extraction fraction with rapid sequential scans due to the short half-life of the tracers. ${ }^{56,57}$ PET is the most expensive of the tomographic modalities for measuring $\mathrm{CBF}$, making it more favorable as a research tool, to study physiology or validate other perfusion techniques, than as a clinical tool. However, recent advances in detector technology and greater availability of radiopharmaceuticals have improved the desirability of PET for clinical use. $^{58}$

\subsection{X-ray techniques}

\subsubsection{Xenon-enhanced computed tomography}

Stable xenon is a radiodense diffusible contrast agent that freely crosses the blood-brain barrier. The Xe-CT procedure begins with a baseline CT scan. Then, sequential CT scans are performed during inhalation of $\sim 30 \%$ xenon. Baseline values are subtracted from each voxel of the xenon enhanced CT images to determine the concentration of xenon in brain tissue and a curve is computed to describe tracer accumulation over time within each voxel. Additionally, the arterial input function (AIF) is estimated by measuring end-tidal xenon concentration. Similar to the PET case of Eq. (18), the fact that the tracer concentration can be measured directly in brain tissue releases the Kety-Schmidt requirement of venous blood measurements, and the equation for Xe concentration in tissue is ${ }^{59}$

$C_{T}^{(\mathrm{Xe})}(t)=\mathrm{CBF} \int_{0}^{t} C_{a}^{(\mathrm{Xe})}(\tau) \mathrm{e}^{-\frac{\mathrm{CBF}}{\lambda}(t-\tau)} \mathrm{d} \tau$,

where $\lambda$ is now the equilibrium tissue-blood partition coefficient for Xe. This form of the Kety-Schmidt equation may be solved with an iterative least squares approach to yield CBF. ${ }^{59}$

Patient motion is a limitation of Xe-CT that can cause artifacts in images. Clinical safety of xenon was assessed in a widespread study, and the authors concluded that Xe-CT has low risk for adverse events. ${ }^{60} \mathrm{CBF}$ was quantified in a study of patients with aneurysmal SAH with a bedside Xe-CT scanner supporting the potential utility of mobile CT scanners in neurointensive care units. ${ }^{61}$ For further clinical applications of CBF measurements in the neuro critical care unit, see Sec. 6.2.

\subsubsection{Perfusion computed tomography}

The bolus tracking methodology for cerebral perfusion computed tomography (PCT) uses the central volume principle (see Sec. 3.1.2), which relates the mean blood transit time $\left(\mathrm{MTT}_{B}\right)$ through a region of interest, the CBV (units: $\mathrm{ml}_{\text {blood }} / g_{\text {tissue }}$ ), and $\mathrm{CBF}^{62}$ The central volume principle is expressed by Eq. (10), which is repeated here

$\mathrm{CBF}=\frac{\mathrm{CBV}}{\mathrm{MTT}_{B}}$.

The PCT procedure begins with a baseline scan with no contrast. Then, an iodinated, nondiffusible contrast agent is injected intravenously. With rapid sequential scanning, time-concentration curves of the contrast-bolus can be constructed. One algorithm used to analyze PCT data to obtain CBV and $\mathrm{MTT}_{B}$, and then CBF from Eq. (21), is the deconvolution algorithm, which is based on the assumption of a hermetically sealed system. ${ }^{63}$

Although $\mathrm{MTT}_{B}, \mathrm{CBV}$, and CBF can be obtained quantitatively, some questions remain about the accuracy and reproducibility of PCT. A correct determination of the AIF is also a challenge that makes absolute quantification of PCT parameters difficult. ${ }^{64}$ Motion artifacts are also a concern and may further limit the accuracy of PCT. For these reasons, relative rather than absolute maps of CBF are often used in PCT.

The biggest impact of PCT has been in imaging of stroke. ${ }^{65}$ Ratios of CBF in brain regions may be used as a quantitative method for interpatient CBF comparisons based on PCT 
measurements. ${ }^{63}$ Computed tomography angiography may be performed in the same imaging session as PCT for anatomic investigation of vessels. ${ }^{66-68}$ See Sec. 6.2 for additional clinical applications of perfusion CT.

\subsection{Magnetic Resonance Imaging}

\subsubsection{Dynamic susceptibility contrast, or perfusion-weighted MRI}

Similar to the case of the Kety-Schmidt method (Sec. 3.2.1), MRI measurements of CBF with dynamic susceptibility contrast (DSC-MRI), also referred to as perfusion-weighted imaging or bolus-tracking MRI, require the injection (intravenous in this case) of a bolus of contrast agent (Gadolinium-based). A key difference with respect to the Kety-Schmidt method, however, is that the MR contrast agents used for DSC-MRI do not diffuse through the blood-brain barrier and remain in the vascular space. The source of contrast for DSC-MRI measurements is the magnetic field gradient between the capillaries and the surrounding tissue, which results from the presence of the intravascular paramagnetic contrast agent. This field gradient leads to a spin dephasing of the water in the bloodstream (i.e., a decrease in the transverse relaxation time $T_{2}$ and a corresponding drop in the MR signal) that depends on the local CBF. ${ }^{69}$ Because such spin dephasing occurs during the relatively short transit time of the contrast bolus (in the order of several seconds), the fast technique of echo-planar imaging is ideally suited for DSC-MRI.

The fact that DSC-MRI uses nondiffusible tracers means that the tracer bolus stays in the vasculature, and its residence time in a given brain VOI, described by the probability density function $h(t)$ introduced in Sec. 3.1.2, coincides with the blood transit time in that volume. As expressed by Eq. (7), the probability that a tracer introduced in the brain VOI at time 0 is still present in the VOI at a later time $t$ is given by $R(t)=1-\int_{0}^{t} h(\tau) \mathrm{d} \tau$. Therefore, the tracer concentration (per unit tissue volume) in the VOI at any time $t\left[C_{T}(t)\right]$ is equal to the temporal convolution between $R(t)$ and the rate at which the tracer enters the VOI per unit tissue volume, $\mathrm{CBF} \times C_{a}(t)$, where $C_{a}(t)$ is the arterial tracer concentration (also referred to as the AIF). Based on these observations, the CBF in the VOI satisfies the following equation 70

$C_{T}(t)=\mathrm{CBF} \int_{0}^{t} C_{a}(\tau) R(t-\tau) \mathrm{d} \tau$,

which coincides with Eq. (5), with the integral carried out for times that follow the introduction of the bolus at $t=0$, as was done in Eq. (18) (in fact, $C_{a}$ is 0 for $t<0$ ). An additional factor $1 / k_{H}$ (where $k_{H}$ is a dimensionless factor related to the difference in hematocrit in small and large blood vessels) may be introduced on the right-hand-side of Eq. (22) to take into account the different cell/plasma volume ratio (only the plasma volume is accessible to the tracer) in the microcirculation and in large blood vessels. ${ }^{71}$ Equation (22) shows that an absolute measurement of CBF with DSC-MRI requires the convolution of $C_{a}(\tau)$ (which is measured as close as possible to the tissue VOI) with the residence probability $R(t)$, which depends on the properties of the local microcirculation. This problem has been addressed by deriving analytical expressions of $R(t)$ on the basis of a model of the microvascular hemodynamics (model-dependent methods), or by solving Eq. (22) for $\mathrm{CBF} \times$ $R(t)$ in every image pixel (model-independent methods). ${ }^{72}$ A study on nine healthy human subjects (28 to 42 years old) with perfusion-weighted MRI reported a global average CBF of $55 \pm 5 \mathrm{ml} /(100 \mathrm{~g} \mathrm{~min}){ }^{73}$

Alternatively, instead of an absolute measurement of CBF, one may perform qualitative $\mathrm{CBF}$ measurements on the basis of summary parameters related to the negative peak of the MR signal during the bolus transit in the brain VOI (time to peak, bolus arrival time, peak width, peak area, and so on). ${ }^{71}$

As of today, perfusion-weighted MRI remains mostly an investigational tool that has yet to achieve widespread clinical use. However, the practical and technical limitations that have limited the clinical use of cerebral perfusion assessment with MRI can be overcome to lead to a potentially powerful clinical tool. $^{74,75}$

\subsubsection{Arterial spin labeling}

Contrary to DSC-MRI, presented in the previous section, the MRI technique of arterial spin labeling (ASL) does not use an extrinsic contrast agent and is therefore completely noninvasive. ASL is based on magnetically labeling water in major arteries that perfuse the brain. Such labeling is achieved by inverting the blood magnetization with a combination of a radiofrequency pulse and a field gradient in the direction of BF, thus defining an inversion plane at the base of the brain. Therefore, ASL uses a freely diffusible tracer in the form of magnetically labeled water, which alters the brain tissue magnetization by exchanges with tissue water. The basic concept for ASL is illustrated in Fig. 4, which shows the labeling and imaging planes. In its original realization, ASL uses a series of radiofrequency pulses to continuously saturate blood water spins, and this technique is referred to as continuous ASL (CASL). ${ }^{76-78}$ Because the change in tissue magnetization due to the magnetically labeled water is dependent upon BF, CBF measurements with continuous ASL are based on the brain tissue magnetization before the application of the inversion pulse $\left(M_{T}^{0}\right)$ and after steady-state conditions are reached $\left(M_{T}^{s s}<M_{T}^{0}\right)$. The expression for CBF used by continuous ASL is as follows ${ }^{76}$

$\mathrm{CBF}=\frac{\lambda}{T_{1 \mathrm{app}}}\left(1-\frac{M_{T}^{s S}}{M_{T}^{0}}\right)$,

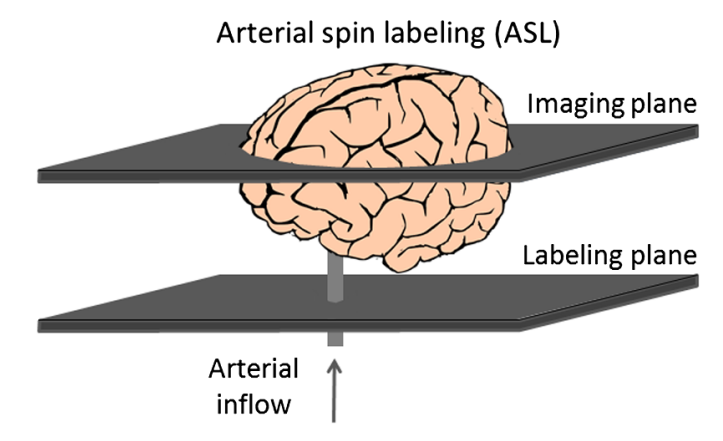

Fig. 4 Arterial spin labeling (ASL). Schematic representation of the basic approach for blood flow measurement with arterial spin labeling MRI. The water in the arterial inflow to the brain is magnetically tagged in the labeling plane. In the imaging plane, the change in tissue magnetization is directly related to blood flow and yields a measure of CBF. 
where $\lambda$ is the tissue-blood partition coefficient for water $\left(\mathrm{ml}_{\text {blood }} / g_{\text {brain }}\right)$ and $T_{1 \text { app }}$ is the time constant describing the decay of tissue magnetization from $M_{T}^{0}$ to $M_{T}^{s s}$.

The use of a single inversion radiofrequency pulse (typically $\sim 10 \mathrm{~ms}$ in duration) and a labeling region that is closer to the imaging slice result in pulsed ASL (PASL) [for a review, see Ref. 79]. PASL addresses two problems of CASL: magnetization transfer effects that alter the tissue magnetization independent of flow effects (due to the long application of the series of radiofrequency pulses), and the loss of spin labels as blood travels from the labeling plane to the imaging slice. ${ }^{71}$ PASL is implemented by a number of different techniques with creative acronyms such as EPISTAR (echo planar imaging and signal targeting with alternating radiofrequency), ${ }^{80}$ PICORE (proximal inversion with a control for off-resonance effects), ${ }^{81}$ FAIR (flow-sensitive alternating inversion recovery), ${ }^{82}$ and QUIPSS II (quantitative imaging of perfusion using a single subtraction, version II) ${ }^{83}$ These various PASL techniques implement various schemes for the acquisition of the tag and control images, but the concept is always the same: measuring local CBF by quantifying the decrease in tissue magnetization caused by the inflow of labeled arterial blood that carries a negative magnetization (i.e., magnetization that is flipped by $180 \mathrm{deg}$ ).

\subsection{Ultrasound techniques}

\subsubsection{Transcranial Doppler ultrasound}

TCD ultrasound provides noninvasive measurements of the BF velocity (units: $\mathrm{cm} / \mathrm{s}$ ) in the basal brain arteries (MCA, proximal anterior cerebral arteries and posterior cerebral arteries), ${ }^{84}$ most commonly in the left or right MCAs. The ultrasound transducer is placed in a subject-specific ultrasonic window in the temporal region above the zygomatic arch, also called the transtemporal window, which is shown in Fig. 5(a). ${ }^{85}$ In Fig. 5(b), it is shown how a TCD ultrasound probe placed at this transtemporal window can measure flow velocity in the MCA. ${ }^{85}$ The basic principle is that an ultrasound wave at frequency $f$ experiences a Doppler frequency shift $(\Delta f)$ as it gets reflected by the red blood cells that are moving at speed $v_{B}$. The relationship between $v_{B}$ and $\Delta f$ is as follows: ${ }^{86}$

$v_{B}=\frac{c_{s}}{2 f \cos (\theta)} \Delta f$ (a)

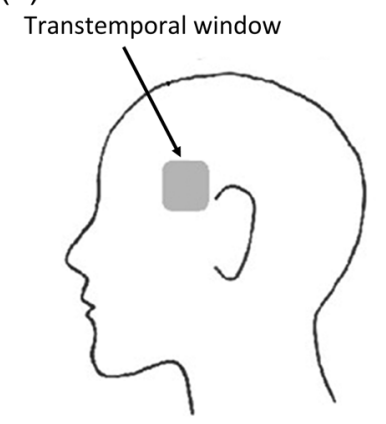

(b)

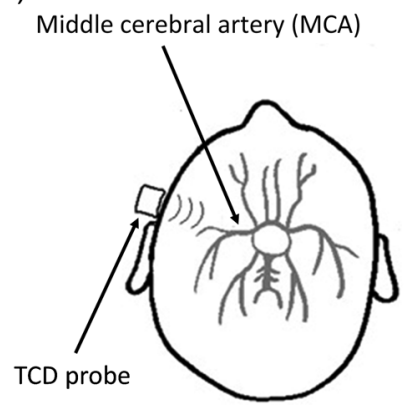

Fig. 5 Transcranial Doppler ultrasound. (a) The location of the transtemporal window on the left side of a human subject. (b) A transcranial Doppler (TCD) probe placed against the left transtemporal window has access to the left MCA for measuring blood flow velocity. Reproduced from Ref. 85 with permission. where $c_{s}$ is the speed of ultrasound in tissue, and $\theta$ is the angle between the direction of propagation of ultrasound and the direction of the blood vessel. The uncertainty in the angle $\theta$ complicates accurate measurements of $v_{B}$. Furthermore, the variability in blood vessel diameter introduces an additional uncertainty in translating the speed of BF (in units of $\mathrm{cm} / \mathrm{s}$ ) into a measure of volumetric $\mathrm{BF}$ (in units of $\mathrm{ml} / \mathrm{s}$ ) in the blood vessel. Even a good estimate of volumetric BF would still require knowledge of the brain mass perfused by the artery in order to estimate an absolute CBF. For these reasons, TCD provides only a relative index of $\mathrm{CBF}$.

A further refinement of TCD is transcranial color Doppler sonography, which combines $2 \mathrm{D}$ (B-mode) images of the brain (through the intact temporal plate) with a color-coded representation of BF with Doppler ultrasound. ${ }^{87}$

\subsubsection{Transit-time ultrasonic flowmetry}

An ultrasound technique to measure flow in an isolated blood vessel consists of placing it within an ultrasonic perivascular flow probe. This technique is applicable only invasively in a surgical setting. The perivascular flow probe features two ultrasound transducers, placed longitudinally on one side of the blood vessel, and an acoustic reflector on the opposite side of the blood vessel. The probe measures the transit times of ultrasound from one transducer to the other when traveling upstream $\left(t_{12}\right)$ and downstream $\left(t_{21}\right)$ to the BF. The difference between the upstream and downstream transit times $\Delta t=t_{12}-t_{12}>0$ is directly proportional to the volumetric $\mathrm{BF}$ in the blood vessel (VBF, units: $\mathrm{ml} / \mathrm{s}$ ), as opposed to the $\mathrm{BF}$ velocity $(\mathrm{cm} / \mathrm{s})$ measured by Doppler ultrasound ${ }^{88}$

$\mathrm{VBF}=\frac{k \pi D c_{s}^{2}}{16 \cot (\theta)} \Delta t$

In Eq. (25), $k$ is a correction factor related to the blood velocity distribution in the blood vessel, $D$ is the blood vessel diameter, $c_{s}$ is the speed of ultrasound in blood, and $\theta$ is the angle between the direction of propagation of ultrasound and the blood vessel direction. This technique has been used to quantify the BF in individual vessels during aneurysm surgery. ${ }^{89}$ The translation of the volumetric flow within a blood vessel [as given in Eq. (25)] into CBF would require the estimation of the brain mass that is perfused (for an artery) or drained (for a vein) by this blood vessel (similar to what was done in Eq. (14) to translate jugular flow into $\mathrm{CBF}$ ). In practice, VBF can be taken only as a relative measure of CBF.

\subsection{Thermal Diffusion Flowmetry}

Thermal diffusion flowmetry (TDF), which is also referred to as heat or thermal clearance method, measures absolute BF in the brain cortex or in the white matter. ${ }^{90}$ This technique can measure a spherical volume of about 20 to $30 \mathrm{~mm}^{3}$ surrounding the probe, which is placed within brain tissue. ${ }^{91}$ TDF provides continuous and invasive bedside monitoring of local CBF.

TDF is based on the principles of thermal transfer via the conductive properties of brain tissue and the convective effects of BF. A thermistor can measure temperature via changes in electrical resistance. A TDF probe consists of two thermistors - a passive one that measures brain temperature and is maintained at this temperature (neutral plate) and an active one held at a slightly higher temperature (heated plate). A schematic 


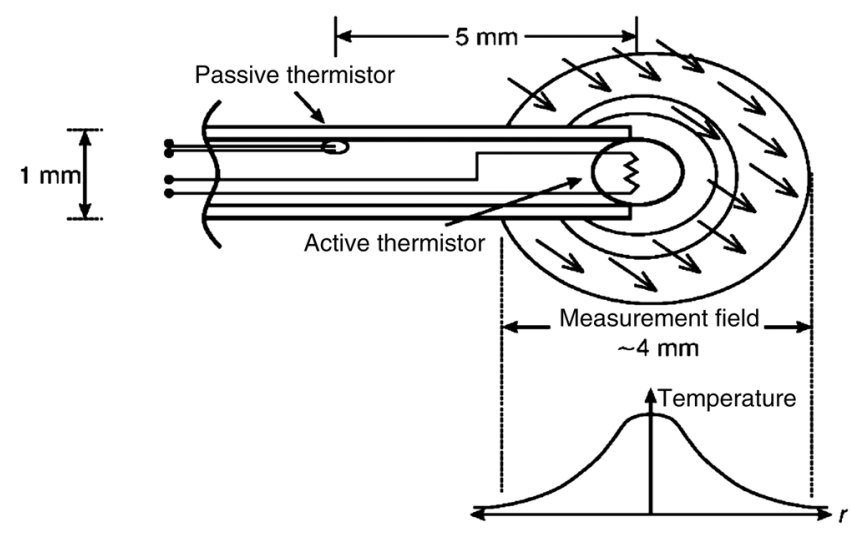

Fig. 6 Thermal diffusion flowmetry. Schematic diagram of the thermal diffusion probe (TDP) showing the active, heated thermistor at the probe tip, which produces a thermal measurement field in the surrounding tissue. The size of the thermal measurement field is dependent on the tissue thermal properties and the perfusion: high perfusion produces a smaller thermal field. The diameter of the field $(2 r)$ is approximately $4 \mathrm{~mm}$ for typical values of thermal properties and perfusion. The passive thermistor, mounted $5 \mathrm{~mm}$ proximal to the probe tip, monitors the tissue baseline temperature variations. Reproduced from Ref. 92 with permission of Springer.

diagram of the TDF probe is shown in Fig. $6 .{ }^{92}$ Thermal power is dissipated from the heated plate due to the thermal conduction of the tissue matter and the convection of CBF. These two components of heat transfer need to be separated in order to compute $\mathrm{BF}$ as an absolute value. Vajkoczy et al. ${ }^{93}$ determined the conductive properties, assumed to be constant, of the tissue surrounding the probe from the initial rate of propagation of the thermal field. For details on the thermal model, see Ref. 94.

For safety purposes, TDF is not applied when the patient has a high fever in order to avoid further heating of the tissue. Placement of the probe near large vessels may also affect reliability. ${ }^{90}$ If the probe is not properly placed or if it moves, the assumption that the heat-conducting properties of tissue matter are constant is no longer true. ${ }^{95}$

TDF has been used to measure the effectiveness of CA following TBI. ${ }^{96}$ Other examples of applications are to predict patient outcome after brain injury and to detect vasospasm in patients with $\mathrm{SAH} .{ }^{90}$

\subsection{Optical techniques}

\subsubsection{Laser Doppler flowmetry}

LDF is a technique for continuous monitoring of relative changes in $\mathrm{CBF}^{97,98} \mathrm{LDF}$ samples a superficial volume of 1-2 $\mathrm{mm}^{3}$ in tissue ${ }^{91}$ so that, while it is conceptually noninvasive, it requires removal of scalp and skull for access to the human brain, thus rendering it an invasive technique for human studies. The LDF instrument consists of a fiber-optic probe and uses monochromatic light in the range of 670 to $810 \mathrm{~nm}$. The movement of red blood cells induces a Doppler shift in the light that is proportional to the velocity and number of red blood cells. ${ }^{99}$ A photodetector measures the scattered light that has been Doppler shifted and these measurements are translated to a time-varying voltage that is proportional to changes in CBF. LDF is the Fourier counterpart to DCS, covered in the next section, although these two techniques do not tend to quantitatively agree due to differences in modeling assumptions. ${ }^{100}$
LDF may suffer from artifacts from patient motion and probe displacement. ${ }^{90}$ Clinically, LDF has been used for assessing autoregulation, $\mathrm{CO}_{2}$ reactivity, and responses to therapeutic interventions. ${ }^{91,101}$ LDF has also been used in studies in changes of CBF such as for research on ischemic stroke, cortical spreading depression, and neurovascular coupling. ${ }^{102}$

\subsubsection{Diffuse correlation spectroscopy}

DCS measures relative changes in BF over much greater tissue volumes than LDF, allowing for noninvasive measurements of the microvasculature of superficial brain cortical regions. ${ }^{103}$ It is a safe, noninvasive technique that can be used continuously at the bedside. Because it uses near-infrared (NIR) light, it has similar resolution and penetration depth as near-infrared spectroscopy (NIRS) methods covered in Secs. 3.8.3 and 3.8.4.

A typical DCS system consists of a single-wavelength, NIR, continuous-wave light source with a long coherence length $(\sim 10 \mathrm{~m})$, multimode source fibers with diameters of $\sim 1 \mathrm{~mm}$, single-mode optical fibers for light detection, single-photon counting avalanche photodiodes, and an autocorrelator. The DCS probe is placed against the surface of the subject's head, and NIR light propagates into the brain. The light is scattered by static scatterers as well as by moving red blood cells. The light then reaches the tissue surface and is measured by the optical detectors. The autocorrelator measures the intensity autocorrelation function of the detected photons. A schematic of a typical DCS system is shown in Fig. 7(a). ${ }^{104}$ The normalized intensity auto-correlation function $\left(g_{2}\right)$ is related to the normalized electric field correlation function $\left(g_{1}\right)$ by the Siegert relation. With this relation, the measurements from the autocorrelator can be fit to solutions of the correlation diffusion equation ${ }^{105}$ in order to determine values for $D_{B}$, the effective Brownian diffusion coefficient of the moving scatterers, and $\beta$, a parameter determined by the optical set up of the experiment. From these fitted values, a blood flow index (BFI [units: $\mathrm{cm}^{2} / \mathrm{s}$ ]) can be determined as

$\mathrm{BFI}=\alpha D_{B}$,

where $\alpha$ is the ratio of moving scatterers to the total number of scatterers in the sample. ${ }^{103} \mathrm{BFI}$ is often represented as percent changes relative to a baseline value, in which case it is termed the relative blood flow index (rBFI). Example $g_{2}$ curves based on intensity fluctuations [Fig. 7(b)] are shown in Fig. 7(c) for baseline $\mathrm{CBF}$ (normocapnia during room air breathing) and for increased CBF (hypercapnia during $\mathrm{CO}_{2}$ inhalation). ${ }^{104}$

The DCS system probes the skin, skull, and CSF, in addition to the tissue region of actual interest, the brain. Similar to the NIRS methods, the effects of superficial, extracerebral layers can be suppressed with special techniques. ${ }^{106}$ The DCS measurements of relative changes in BF have been convincingly validated, but quantification of absolute $\mathrm{CBF}$ remains a challenge. ${ }^{104}$

\subsubsection{Near-infrared spectroscopy}

NIRS is a noninvasive technique whose optical probes are placed on the subject's scalp, through hair if present, for measurements on the cerebral cortex. NIRS has been applied to functional brain studies ${ }^{107-109}$ as well as a variety of pilot clinical studies $^{110}$ aimed at brain monitoring during anesthesia, ${ }^{111}$ after brain injury, ${ }^{112,113}$ or during electroconvulsive therapy. ${ }^{114}$ The 

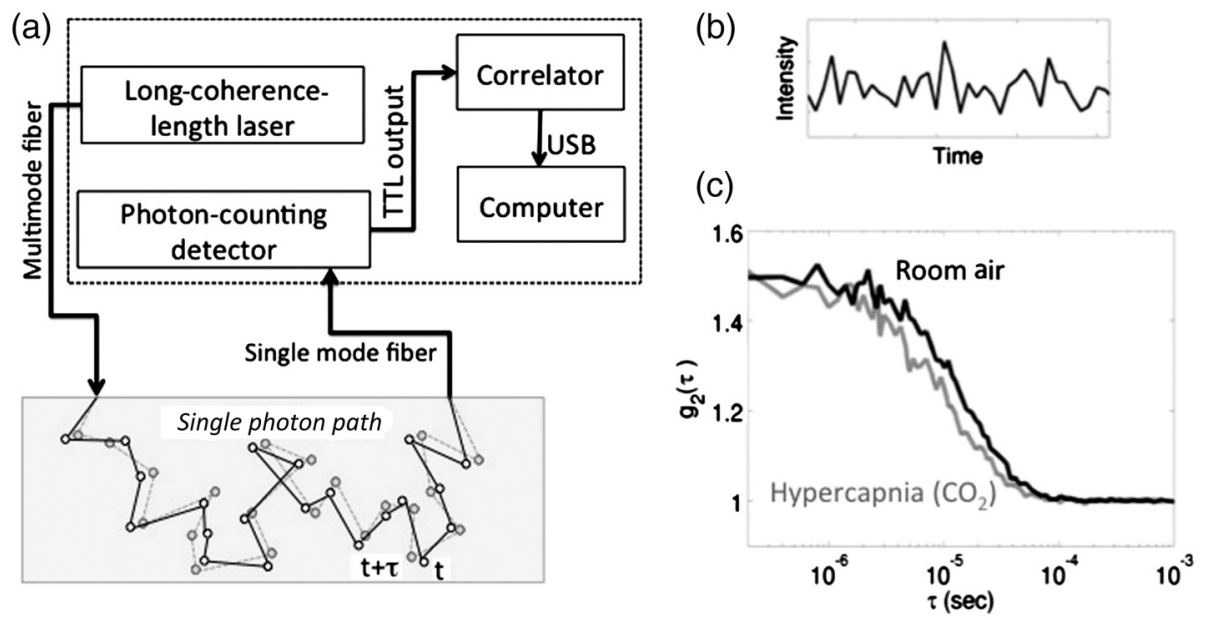

Fig. 7 Diffuse correlation spectroscopy (DCS). (a) Schematic of typical DCS instrumentation that consists of a long-coherence length source coupled to a multimode fiber for light delivery to the tissue, photon-counting detector(s), and an auto-correlator board that computes the intensity of the autocorrelation function, $g_{2}(\tau)$, based on photon arrival times (b), (c) Sample $g_{2}(\tau)$ curves obtained over the frontal cortex in a subject under baseline conditions (black) and under hypercapnia ( $3 \%$ inspired carbon dioxide, gray). The increased decay rate of $g_{2}(\tau)$ during hypercapnia reflects the increase in CBF by vasodilation. Reproduced from Ref. 104 with permission of the Society of Photo Optical Instrumentation Engineers (SPIE).

spatial resolution is on the order of $1 \mathrm{~cm}$, and the maximum tissue depth that can be probed is $2-3 \mathrm{~cm}$. NIRS is applicable at the bedside and can provide continuous monitoring. It is a diffuse optical method that is sensitive to the tissue concentrations of oxyhemoglobin, $\left[\mathrm{HbO}_{2}\right]$, and deoxyhemoglobin, [Hb], due to the differential absorption of NIR light of the oxygenated and deoxygenated heme group. The sum of $\left[\mathrm{HbO}_{2}\right]$ and $[\mathrm{Hb}]$ yields the total hemoglobin concentration, [HbT], and the ratio $\left[\mathrm{HbO}_{2}\right] /[\mathrm{HbT}]$ yields the oxygen saturation of hemoglobin in brain tissue, $\mathrm{StO}_{2}$, also called $\mathrm{ScO}_{2}$ to specify cerebral tissue. Because hemoglobin is confined to the red blood cells and the vast majority of oxygen in blood is bound to hemoglobin, [HbT] is proportional to $\mathrm{CBV}$, whereas $\mathrm{ScO}_{2}$ and the concentration difference $[\mathrm{HbD}]=\left[\mathrm{HbO}_{2}\right]-[\mathrm{Hb}]$ are indicators of the balance between oxygen supply and oxygen demand in tissue. Such a balance is determined by a number of factors, including BF, blood volume, metabolic rate of oxygen, capillary density, and hematocrit. Even though $[\mathrm{HbD}]$ and $\mathrm{ScO}_{2}$, which provide a measure of intravascular oxygenation, are not solely determined by $\mathrm{CBF}$, they are nevertheless linked to CBF. In particular, $[\mathrm{HbD}]$ was found to correlate with $\mathrm{CBF}$ in piglets, ${ }^{115,116}$ in infants undergoing surgery, ${ }^{117}$ and in critically ill patients. ${ }^{118}$ Of course, $[\mathrm{HbD}]$ and $\mathrm{ScO}_{2}$ can provide only relative measurements of $\mathrm{CBF}$, and they rely on the assumption that other physiological factors, besides $\mathrm{CBF}$, do not contribute significantly to their values. Furthermore, the details of the [HbD] and $\mathrm{ScO}_{2}$ temporal dynamics may not accurately represent the $\mathrm{CBF}$ dynamics.

Absolute measurements of CBF have been performed with tracer tracking techniques. The two main tracers have been oxyhemoglobin (endogenous) and indocyanine green (ICG) (exogenous). In the oxyhemoglobin method, the inspired oxygen fraction is first lowered and then sharply increased so that the resulting sudden burst in oxyhemoglobin concentration in blood acts as a bolus. ${ }^{19,120}$ For the first few seconds of measurement with NIRS, the venous outflow of the oxygen bolus is assumed to be zero. In other words, NIRS measurements are performed at times $(t)$ that are shorter than the bolus transit time in the measured volume. Under these conditions, the Fick principle yields Eq. (5), or Eq. (22), with a tracer resident probability $R(t)=1$, leading to the following expression for $\mathrm{CBF}$ in terms of the change in $\left[\mathrm{HbO}_{2}\right]$, the intravascular tracer, that is observed in a time interval $\Delta t$ that is shorter than MTT $^{119}$

$\mathrm{CBF}=k \frac{\Delta\left[\mathrm{HbO}_{2}\right]}{\operatorname{ctHb} \int_{0}^{\Delta t} \Delta \mathrm{SaO}_{2}(t) \mathrm{d} t}$,

where $\Delta\left[\mathrm{HbO}_{2}\right]$ is the oxyhemoglobin concentration change measured with NIRS [units: $\mathrm{mol}_{\mathrm{HbO} 2} / \mathrm{ml}_{\text {tissue }}$ ] over time $\Delta t, \mathrm{ctHb}$ is the blood concentration of hemoglobin [units: $\mathrm{mol}_{\mathrm{HbT}} / \mathrm{ml}_{\text {blood }}$ ], $\Delta \mathrm{SaO}_{2}$ is the change in arterial saturation (units: $\mathrm{mol}_{\mathrm{HbO} 2} / \mathrm{mol}_{\mathrm{HbT}}$ ), measured noninvasively with a pulse oximeter, and $k$ is a constant (units: $\mathrm{ml}_{\text {tissue }} / g_{\text {tissue }}$ ) that takes into account the small-to-large vessel hematocrit ratio and the mass density of brain tissue. This is a noninvasive method to measure absolute CBF. Even though oxygen is a freely diffusible tracer, one can consider the rate of oxygen diffusion from blood to brain (i.e., $\mathrm{CMRO}_{2}$ ) to be accomplished by the baseline hemoglobin flow, so that the bolus of $\Delta\left[\mathrm{HbO}_{2}\right]$, i.e., the extra amount of oxyhemoglobin, may be treated as a nondiffusible tracer in Eq. (27).

The ICG method requires an intravenous bolus injection of the contrast agent. ${ }^{121}$ Under physiological conditions, ICG binds to plasma proteins (mostly albumin) and does not cross the blood brain barrier, so that it remains confined to the vascular space. Therefore, NIRS measurements can be analyzed with a similar approach to the oxyhemoglobin method. ${ }^{122}$ In these early approaches, the arterial concentration of ICG was measured invasively by arterial catheterization. Another approach is based on the central volume principle, or on the general form of Eq. (5) without requiring that NIRS measurements be performed before any venous ICG outflow takes place. ${ }^{123}$ The tissue concentration of ICG is measured with NIRS and the ICG arterial concentration is measured with a noninvasive, 
dye-sensitive oximeter. Deconvolution between arterial and tissue ICG concentrations is performed in order to recover the resident probability function, $R(t)$ [see Eq. (5)], from which $\mathrm{MTT}_{B}, \mathrm{CBF}$, and CBV can be computed. ${ }^{123}$ Instead of doing multiple injections, the NIRS technique can be used to compute a baseline value for CBF in absolute units from a single bolus injection. This baseline value can then be combined with a relative and continuous measure of $\mathrm{CBF}$, such as that provided by $[\mathrm{HbD}] .{ }^{124}$ In another approach, DCS (see Sec. 3.8.2) was used to obtain quantitative relative $\mathrm{CBF}$ changes in combination with ICG and time-resolved NIRS measurements. ${ }^{125}$

One of the challenges with NIRS for measuring CBF is that measurements are contaminated by extracerebral BF. ${ }^{126}$ In fact, with typical methods to measure changes in oxyhemoglobin concentration (modified Beer-Lambert law), $\Delta\left[\mathrm{HbO}_{2}\right]$ in Eq. (27) represents a weighted average of the concentration changes occurring in extracerebral and cerebral tissue. Twolayer diffusion models, short and long source-detector separations, and other approaches for separating extracerebral BF contributions are an active area of research in the field.

\subsubsection{Coherent hemodynamics spectroscopy}

Coherent hemodynamics spectroscopy (CHS) is a technique that we have recently developed to translate hemodynamic cerebral measurements into physiological quantities, including CBF. ${ }^{127}$ This technique can be applied to NIRS data, in which case it has the same spatial resolution and reaches the same tissue depth as described in the NIRS section. CHS has the potential to provide noninvasive and continuous bedside monitoring of absolute CBF. ${ }^{128}$

CHS features two alternative methods for measuring physiological parameters. In the first method, operating in the frequency-domain, techniques such as paced breathing or cyclic thigh cuff inflations induce oscillations in MAP, which in turn result in oscillations of cerebral oxy- and deoxyhemoglobin concentrations (indicated with $O$ and $D$, respectively, in CHS) that are highly coherent with MAP. The phase and amplitude of ratios of $O$ and $D$ oscillations are combined to generate CHS spectra that are fit with a hemodynamic model, ${ }^{127}$ whose fitting parameters relate to microvascular flow and volume. ${ }^{129}$ In the second method, operating in the time domain, thigh cuffs are rapidly released after a 2 min arterial occlusion, thereby inducing a transient decrease in MAP and corresponding transient changes in cerebral oxy- and deoxyhemoglobin concentrations, which are fitted with the time-domain version of the hemodynamic model. ${ }^{130}$ Both methods yield several parameters, two of which can be used to compute an absolute value of baseline CBF. These two parameters are the mean blood transit time in the capillaries, $t^{(c)}$, and the capillary blood volume fraction, $\mathcal{F}^{(c)} \mathrm{CBV}_{0}^{(c)} / \mathrm{CBV}_{0}$, where $\mathcal{F}^{(c)}$ is the Fåhraeus factor that takes into account the hematocrit reduction in the capillaries, $\mathrm{CBV}_{0}$ is the baseline $\mathrm{CBV}$ (expressed here in $\mathrm{ml}_{\text {blood }} / \mathrm{ml}_{\text {tissue }}$ ), and $\mathrm{CBV}_{0}^{(c)}$ is the capillary blood volume ( $\mathrm{ml}$ of capillary blood per $\mathrm{ml}$ of tissue). From the central volume principle [Eq. (10)] applied to the capillary compartment, the baseline cerebral blood flow, $\mathrm{CBF}_{0}$, can be written as follows: ${ }^{128}$

$$
\mathrm{CBF}_{0}=\frac{1}{\rho_{T}} \frac{\mathcal{F}^{(c)} \mathrm{CBV}_{0}^{(c)}}{t^{(c)}}=\frac{1}{\rho_{T}} \frac{T_{0}}{\mathrm{ctHb}} \frac{\frac{\mathcal{F}^{(c)} \mathrm{CBV}_{0}^{(c)}}{\mathrm{CBV}_{0}}}{t^{(c)}}
$$

where $\rho_{T}$ is the brain tissue density, $T_{0}$ is the baseline total hemoglobin concentration (measurable with time-resolved $\mathrm{NIRS}$ ), and ctHb is the concentration of hemoglobin in blood (measurable from a blood test). In Eq. (28), we have used the fact that $T_{0}=\mathrm{ctHb} \mathrm{CBV}_{0}$.

In addition to absolute measurements of $\mathrm{CBF}_{0}$, $\mathrm{CHS}$ can also measure time traces of relative $\mathrm{CBF}$ changes, indicated with lower-case notation, $\operatorname{cbf}(t)$ (i.e., $\operatorname{cbf}(t)=[\mathrm{CBF}(t)-$ $\left.\left.\mathrm{CBF}_{0}\right] / \mathrm{CBF}_{0}\right) .{ }^{131}$ Because perturbations of $\mathrm{CBF}$ and metabolic rate of oxygen cannot be distinguished in NIRS measurements, ${ }^{132}$ we assume a constant metabolic rate of oxygen. Under this condition, the frequency domain version of our hemodynamic model yields the following expression for the Fourier transform of cbf, which can be obtained experimentally from the Fourier transform of the $O$ and $D$ time traces: ${ }^{131}$

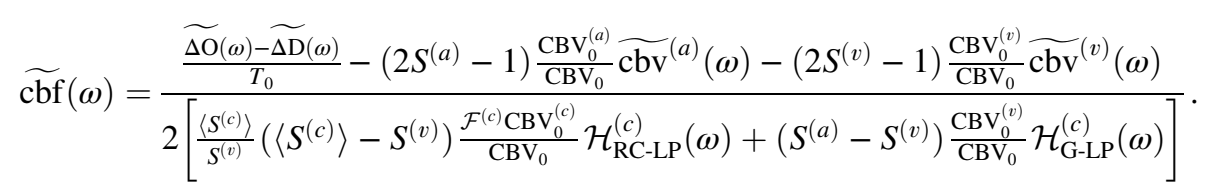

In Eq. (29), the tilde indicates Fourier transformation, cbv is relative blood volume changes, $S$ is the oxygen saturation of hemoglobin, and parameters specific to a particular vascular compartment are indicated with a superscript [ $(a)$ for arterial, $(c)$ for capillary, and $(v)$ for venous]. The two filters are notated with $\mathcal{H}$ where $\mathcal{H}_{\mathrm{RC}-\mathrm{LP}}^{(c)}$ is an RC low-pass filter describing the capillary compartment, and $\mathcal{H}_{\mathrm{G}-\mathrm{LP}}^{(v)}$ is a Gaussian low pass filter describing the venous compartment. The inverse Fourier transform of Eq. (29) yields the time trace of relative cerebral blood flow, $\operatorname{cbf}(t)$. With the results of Eqs. (28) and (29), the time trace of absolute cerebral blood flow $\operatorname{CBF}(t)$ can be written as follows:

$\operatorname{CBF}(t)=\mathrm{CBF}_{0}[1+\operatorname{cbf}(t)]$
The first clinical application of CHS revealed a significantly longer cerebral capillary transit time in hemodialysis patients $(1.1 \pm 0.2 \mathrm{~s})$ with respect to healthy subjects $(0.5 \pm 0.1 \mathrm{~s}),{ }^{133}$ and this longer capillary transit time corresponds to a lower baseline $\mathrm{CBF}_{0}{ }^{128}$

\section{Methods to measure arterial blood pressure}

We describe four of the most common methods for ABP measurements: arterial line, finger plethysmography, sphygmomanometry, and tonometry. ${ }^{18}$ For each technique, we specify whether it is invasive or noninvasive, and whether it allows for continuous or discrete-time monitoring of ABP. 


\subsection{Arterial line}

The arterial line is an invasive beat-to-beat method to measure $\mathrm{ABP}$ and is considered the gold standard. The main components of an arterial line are an intra-arterial cannula (to access an artery, usually the radial artery) a fluid filled tubing (to provide hydraulic coupling between blood in the cannula and the transducer), a transducer (to convert changes in blood pressure into electrical signals), a processor, an amplifier, and a display. ${ }^{134} \mathrm{~A}$ blood pressure wave is characterized by its first and higher-order harmonics. Usually, in order to have a good reconstruction of the $\mathrm{ABP}$ wave, 8-10 harmonics are needed; therefore, the detection system should be able to "pass" the harmonics up to these orders without altering their amplitude. This is achieved by having a detection system with a natural frequency that exceeds the frequency of the highest harmonic for the highest heart rate one wants to sample. ${ }^{135}$ In other words, the detection system should behave, with respect to the input (i.e., the ABP wave), as a low pass filter having a cutoff frequency with the specified requirements. For example, in order to reproduce pulse rates up to $140 \mathrm{bpm}$ with 10 harmonics, a cutoff frequency greater than $23.3 \mathrm{~Hz}\left[23.3 \mathrm{~Hz}=140 \mathrm{~min}^{-1} \times 1 \mathrm{~min} /(60 \mathrm{~s}) \times 10\right]$ is required. The natural frequency of the system can be increased by reducing the length of the cannula, increasing the diameter of the cannula, or by increasing the viscosity of the fluid filling the tubing. The natural frequency of a system can be measured by using the "fast flush" test. ${ }^{135}$ The detection system should also be damped, because as the frequency of the ABP approaches the natural frequency of the system, the system will resonate and amplify the input signal. Damping of the ABP wave derives mostly from friction in the fluid pathway and from other structural mechanisms in the system (like having a long and narrow compliant tubing). A detection system must be carefully designed in order not to be overdamped or underdamped. If a system is overdamped, the features of the ABP wave are lost, and the systolic $\mathrm{ABP}$ is underestimated while the diastolic $\mathrm{ABP}$ is overestimated. On the contrary, an underdamped system will be fast to respond to ABP changes but more noise will be introduced. Moreover, the systolic ABP will be overestimated and the diastolic ABP will be underestimated. An optimally damped system will balance between speed of response and accuracy. This is usually achieved with a damping coefficient of 0.7 . Optimally damped systems can be obtained by different choices of the natural frequency and the damping parameter. For example, the higher the natural frequency, the larger will be the interval for the damping parameter. ${ }^{136}$

\subsection{Finger photoplethysmography}

This method, also known as the volume clamp method, is a noninvasive technique that allows for continuous monitoring of ABP. This method uses the following components: (a) an inflatable finger cuff; (b) a photoplethysmography sensor built into the finger cuff; and (c) a pressure controller unit. When the finger cuff is not inflated, the plethysmography sensor detects the oscillations of light intensity due to the periodic expansion of the arterioles at the cardiac rate. A pulsating pressure is applied to the finger cuff by means of a fast pneumatic servo system that allows for arterial unloading. In other words, the radius of the arterioles is kept constant in order to achieve zero transmural pressure, and in this condition the temporal trend applied to the cuff pressure equals the temporal trend of ABP. More details on the principles of photoplethysmography can be found in Refs. 137 and 138. Figure 8 illustrates the principle of operation for finger photoplethysmography. ${ }^{139}$

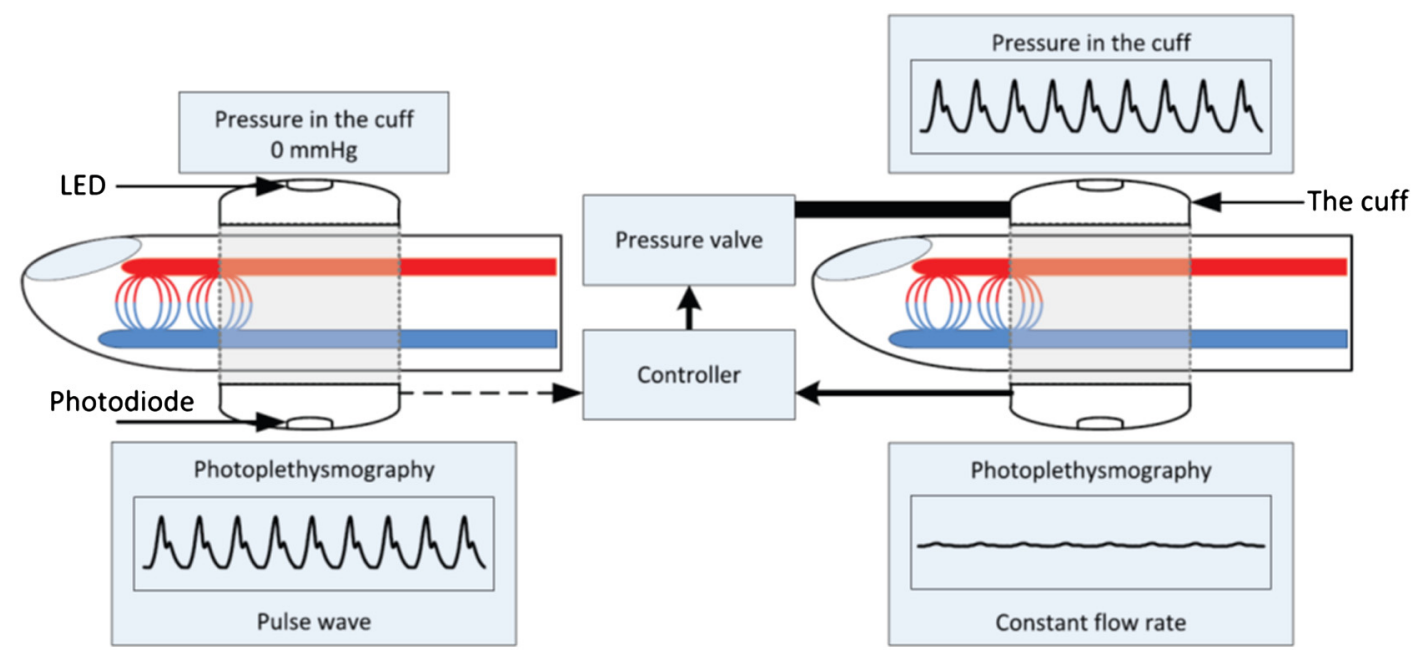

Fig. 8 Finger photoplethysmography. A photoplethysmography sensor, which is an optical system featuring a light emitting diode (LED) and an optical detector (photodiode), is built within a finger inflatable cuff. The pressure in the finger cuff is adjusted by an air control unit placed around the wrist of a subject. When no pressure is applied in the cuff (left panel) the optical signal detected is sensitive to the arterial expansion and contraction at the cardiac rate and shows typical intensity fluctuations due to the systolic and diastolic phases of the heart cycle ("Pulse wave" in the figure). In other words the features of the optical signal reflect the dynamic changes in arterial blood volume. When the finger cuff is inflated by a fast pneumatic servo system to achieve arterial unloading at zero transmural pressure (i.e., the arterial pressure equals the cuff pressure) the optical signal flattens out (right panel). In this situation, there is no change in the arterial blood volume and the blood flows in and out of the arterial compartment are the same ("constant flow rate" in the figure). Reproduced from Ref. 139 with permission. Copyright (C) 2014 Elsevier Masson SAS. All rights reserved. 


\subsection{Sphygmomanometers}

Sphygmomanometry is one of the noninvasive methods that allows for discrete time point monitoring of ABP. It is not a beat to beat method, and therefore only mean, systolic and diastolic ABP can be detected.

Manual: This is an auscultatory method based on listening to the so-called Korotkoff's sounds, ${ }^{139,140}$ which are produced when an arm cuff is inflated to a pressure large enough to occlude the brachial artery and stop BF. After BF is stopped, the cuff is steadily and slowly deflated and five distinct Korotkoff's sounds can be heard. These sounds are detected by using a stethoscope placed over the brachial artery and below a blood pressure cuff. During the deflation, when the cuff pressure reaches systolic pressure, the blood starts to flow again, and a sound is heard (the first phase of the Korotkoff sounds). As the cuff is deflated further, a regime of turbulent flow (when the artery opens and closes) is created in the artery and sounds are still heard. When the cuff pressure is less than the diastolic pressure, the artery is always open and the flow of blood is smooth causing the disappearance of the sounds. Systolic pressure is measured as the pressure at which the first Korotkoff's sound is heard, and diastolic as the pressure at which the sounds disappear. Standard sphygmomanometers are divided into aneroid and mercury sphygmomanometers. ${ }^{141}$

Digital (automatic): Automatic sphygmomanometers are based on the detection of oscillations in the pressure applied to an arm cuff as the pressure is decreased (as in the manual methods) below the systolic pressure. Instead of using an operator who can hear the Korotkoff's sounds in the deflation phase, this method uses a piezoelectric sensor, electronic components, and microprocessors. Mean ABP corresponds to the maximal oscillations during cuff deflation, and the instrument is most sensitive to these oscillations. However, systolic and diastolic $\mathrm{ABP}$ correspond to the appearing and disappearing of pressure cuff oscillations, respectively, and, therefore, are more difficult to detect. More precisely, systolic ABP is measured by an increase in cuff oscillation when blood first passes the deflating cuff. Diastolic ABP is instead detected when oscillations disappear. Detection of systolic and diastolic ABP requires sophisticated algorithms for the analysis of the temporal trends of the pressure cuff oscillations, with different manufacturers using different algorithms. ${ }^{141,142}$

\subsection{Tonometry}

Tonometry of the radial artery provides a noninvasive assessment of the central pulse pressure waveform (pressure in the aorta). Tonometry of the radial artery is carried out by placing a handheld tonometer (strain gauge pressure sensor) over the radial artery and applying a mild pressure in order to induce a partial occlusion of the artery. The pressure from the radial artery is transmitted from the vessel to the sensor and recorded digitally. A sophisticated algorithm based on the study of the reflection of the central pulse pressure waveform from the smaller resistance vessels permits derivation and calculation of central pressure indices from a peripheral brachial blood pressure. ${ }^{143}$

\subsection{Validation of methods and issues with arterial blood pressure measurements}

In the case of the techniques based on an arm pneumatic cuff, several factors have to be considered in order to obtain an accurate reading of ABP. These factors include the posture of the body, and especially of the arm, and the cuff size. ${ }^{141}$ Moreover, auscultatory methods are operator dependent, and for this reason more and more electronic devices to monitor blood pressure are being used at home. Finger photoplethysmography seems a reliable method to measure the beat to beat $\mathrm{ABP}$, and several studies have validated this technique with the arterial line method. ${ }^{137,144}$ Tonometry has also been validated against the invasive arterial line method and good agreement has been found except for its limited capacity to respond to fast variation in $\mathrm{ABP}{ }^{145}$

The general guidelines for testing a new electronic device for ABP measurements have been developed by the British Hypertension Society (BHS) and the Association for the Advancement of Medical Instrumentation (AAMI). Both require three measurements of blood pressure in 85 subjects, chosen to cover a wide range of subjects' ages and blood pressure values. The measurements are taken by trained observers using a gold standard method and the device being tested. The BHS protocol requires that a device must give at least $50 \%$ of readings within $5 \mathrm{mmHg}$, and $75 \%$ of readings within $10 \mathrm{mmHg}$ of the gold standard method. The AAMI requires that the average difference between the tested and gold standard methods does not exceed $5 \mathrm{mmHg}$, with a standard deviation of less than $8 \mathrm{mmHg}$. Therefore, according to these guidelines, it is possible that a new device will pass the test but at the same time it can be in clear disagreement with a gold standard method for several individuals. ${ }^{141}$

\section{Methods to measure cerebral autoregulation}

We conceptually divide the methods to measure CA into direct methods and model-based methods. In direct methods, the metrics for $\mathrm{CA}$ are derived from the relationships between the dynamics of MAP and CBF without any support of physiological and vascular models of cerebral hemodynamics. Signal processing methods featuring various levels of sophistication are applied to the raw data in order to provide metrics of CA. These methods are described in Sec. 5.3. On the contrary, the purpose of model-based methods is twofold: (a) to reach a deeper understanding of CA by providing a physiological and vascular model of the autoregulation process; (b) based on the model, to provide different metrics of CA that can be useful also in clinical settings.

\subsection{Direct methods}

The CA process can be directly observed in invasive studies by measuring the change in blood vessels' diameter in response to changes in MAP. ${ }^{25}$ However, this method cannot be used as a monitor of CA in clinical settings, not only because of its invasiveness, but also because it does not provide a measure of changes in CBF, which is the variable of real interest. Therefore, CA is usually assessed by direct measurements of CBF (or some surrogate) and MAP, and analyzing the relationships of their temporal dynamics by using signal processing methods (see Sec. 5.3). We refer to these methods as direct methods of autoregulation assessment. In principle, any method used to measure CBF can be used to measure CA, as long as it supports the specific requirements of the CA measurements under consideration (static versus dynamic, snapshot versus monitoring, and so on). Most of the current studies on CA (especially those on dynamic CA) are carried out with TCD 
ultrasound, because it is a noninvasive method that allows for continuous bedside monitoring of cerebral blood flow velocity $(\mathrm{CBFv})$ in large arteries, which in many protocols can be considered a reliable substitute of CBF. Most of the data analysis methods covered in this review have been first proposed to analyze TCD data. Other techniques are also currently used to assess CA, like PET, ${ }^{146,147}$ single photon emission computed tomography (SPECT), ${ }^{148}$ magnetic resonance imaging (MRI), ${ }^{149,150}$ and DCS. ${ }^{104}$ Note that in this latter study, ${ }^{104}$ the authors have measured the changes of $\mathrm{CBF}$ in response to posture maneuver (by changing the angle of the bed where the subjects were lying) in healthy subjects and in patients affected by stroke (either typical or paradoxical). The study showed a clear difference between the responses of CBF by the different groups even though the results were not summarized by a quantitative CA index or metric.
Near infrared spectroscopy (NIRS) has been used in a number of studies of CA. ${ }^{118,151-163}$ However, as discussed in Sec. 3.8.3, NIRS cannot measure CBF directly, unless an intravascular bolus is introduced. In its most common form, NIRS measurements of total hemoglobin concentration ([HbT]), tissue oxygen saturation $\left(\mathrm{StO}_{2}\right)$, tissue oxygenation index (TOI), or, more commonly, the difference between oxy- and deoxyhemoglobin concentrations ([HbD]) have been used as surrogates for CBF. More recently, a dynamic NIRS model was used to derive actual CBF measurements from NIRS data. ${ }^{128}$ In Table 3, we present a summary of some recent NIRS studies on CA, including the NIRS parameter used as a surrogate of CBF (if any), the method to derive a CA index, and the applications considered. Among these studies, we have also included a method that combines NIRS and ultrasound by ultrasonically tagging (UT) light. ${ }^{164}$ The UT-NIR method ${ }^{164}$ uses a combination of intensity

Table 3 Recent NIRS studies on cerebral autoregulation (CA).

\begin{tabular}{|c|c|c|c|c|c|c|}
\hline Reference & $\begin{array}{l}\text { NIRS data; } \\
\text { measured quantities }\end{array}$ & $\begin{array}{l}\text { CBF } \\
\text { index }\end{array}$ & Autoregulation index & Application & $n$ & Conclusion \\
\hline 163 & $\mathrm{CW}$ data; $\mathrm{ScO}_{2}$ & & $\begin{array}{l}\text { TFA: } \mathrm{ScO}_{2}-\mathrm{MAP} \\
\text { (phase, gain, coherence) }\end{array}$ & Sepsis & 10 & Poor correlation with TCD \\
\hline 153 & $\mathrm{CW}$ data; $\mathrm{ScO}_{2}$ & & $\begin{array}{l}\text { Pearson correlation } \\
\left(\mathrm{ScO}_{2}-\mathrm{MAP}\right)\end{array}$ & $\begin{array}{l}\text { Hypotensive maneuver } \\
\text { (piglets) }\end{array}$ & 6 & Sensitivity to loss of CA \\
\hline 160 & $\mathrm{CW}$ data; $\mathrm{ScO}_{2}$ & & $\begin{array}{l}\text { TFA: } \mathrm{ScO}_{2}-\mathrm{MAP} \\
\text { (phase, gain, coherence) }\end{array}$ & $\begin{array}{l}\text { Induced changes in } \\
\text { MAP (piglets) }\end{array}$ & 12 & Poor correlation with TCD \\
\hline 164 & $\begin{array}{l}\text { CW data; US signal; } \\
\text { US-modulated } \\
\text { optical signal }\end{array}$ & $\mathrm{CFI}$ & $\begin{array}{l}\text { Pearson correlation } \\
\text { (CFI-MAP) }\end{array}$ & $\begin{array}{l}\text { Cardiopulmonary } \\
\text { bypass }\end{array}$ & 64 & Significant correlation with TCD \\
\hline 166 & $\begin{array}{l}\mathrm{CW} \text { or FD data; } \\
\Delta\left[\mathrm{HbO}_{2}\right], \Delta[\mathrm{Hb}]\end{array}$ & $t^{(c)}$ & $f_{c}^{(A R)}$ & $\begin{array}{l}\text { Hyperventilation, } \\
\text { thigh cuff release }\end{array}$ & 11 & $\begin{array}{l}\text { Higher } f_{c}^{(A R)} \text { during hyperventilation } \\
\text { versus normal respiration }\end{array}$ \\
\hline 157 & $\mathrm{CW}$ data; $\mathrm{ScO}_{2}$ & & $\begin{array}{l}\text { Pearson correlation } \\
\left(\mathrm{ScO}_{2}-\mathrm{MAP}\right)\end{array}$ & $\begin{array}{l}\text { Orthotopic liver } \\
\text { transplantation }\end{array}$ & 33 & Effective measurement of CA \\
\hline 161 & CW data; $\Delta\left[\mathrm{HbO}_{2}\right]$ & & $\begin{array}{l}\text { Phase shift } \\
\Delta\left[\mathrm{HbO}_{2}\right]-\mathrm{MAP}\end{array}$ & $\begin{array}{l}\text { USCAS; paced } \\
\text { breathing }(0.1 \mathrm{~Hz})\end{array}$ & 15 & Spatial assessment of CA \\
\hline 154 & $\begin{array}{l}\text { CW data; } \\
\Delta\left[\mathrm{HbO}_{2}\right], \Delta[\mathrm{Hb}]\end{array}$ & $\Delta[\mathrm{HbD}]$ & $\begin{array}{l}\text { TFA: } \Delta[\mathrm{HbD}]-\mathrm{MAP} \\
\text { (coherence) }\end{array}$ & $\begin{array}{l}\text { Very low birth weight } \\
\text { neonates }\end{array}$ & 90 & Effective monitoring of impaired $\mathrm{CA}$ \\
\hline 158 & CW data; TOI & & $\begin{array}{l}\text { Pearson correlation } \\
\text { (TOI-MAP) }\end{array}$ & Sepsis & 23 & Significant correlation with TCD \\
\hline 151 & $\begin{array}{l}\text { CW data; } \\
\Delta\left[\mathrm{HbO}_{2}\right], \Delta[\mathrm{Hb}]\end{array}$ & $\Delta[\mathrm{HbD}]$ & $\begin{array}{l}\text { TFA: } \Delta[\mathrm{HbD}]-\mathrm{MAP} \\
\text { (coherence) }\end{array}$ & $\begin{array}{l}\text { Very low birth weight } \\
\text { neonates }\end{array}$ & 32 & Effective detection of impaired CA \\
\hline 118 & $\begin{array}{l}\text { CW data; } \\
\Delta\left[\mathrm{HbO}_{2}\right], \Delta[\mathrm{Hb}]\end{array}$ & $\begin{array}{l}\Delta[\mathrm{HbD}] \\
\Delta[\mathrm{HbT}]\end{array}$ & $\begin{array}{l}\Delta[\mathrm{HbD}] / \Delta \mathrm{MAP} \\
\Delta[\mathrm{HbT}] / \Delta \mathrm{MAP}\end{array}$ & Sick neonates & 27 & $\begin{array}{l}\text { Significant correlation with NIRS } \\
\text { bolus measurement of CBF }\end{array}$ \\
\hline 155 & CW data; TOI & & $\begin{array}{l}\text { TFA: TOI-MAP } \\
\text { (coherence) }\end{array}$ & Premature infants & 24 & Effective monitoring of $\mathrm{CA}$ \\
\hline 159 & CW data; TOI & & $\begin{array}{l}\text { Pearson correlation } \\
\text { (TOI-MAP) }\end{array}$ & $\mathrm{SAH}$ & 27 & Significant correlation with TCD \\
\hline
\end{tabular}

$\mathrm{CFI}$ : cerebral flow index; CW: continuous wave; FD: frequency domain; $f_{c}^{(A R)}$ : cutoff frequency for autoregulation; MAP: mean arterial pressure; SAH: subarachnoid hemorrhage; $\mathrm{ScO}_{2}$ : cerebral tissue oxygen saturation; TCD: transcranial Doppler ultrasound; TFA: transfer function analysis; $t^{(c)}$ : capillary transit time; TOI: tissue oxygenation index; US: ultrasound; USCAS: unilateral severe carotid artery syndrome. 
data from NIRS and ultrasound data to define a cerebral flow index (CFI), which is sensitive to the changes in $\mathrm{BF}$ velocity. This method is conceptually similar to the autocorrelation method of DCS, but achieves spatial selectivity by ultrasonic tagging of light. ${ }^{165}$ In Table 3, we have also included a model-based NIRS study (CHS) that is described in Sec. 5.2.2. ${ }^{166}$ Table 3 is organized as follows: the first column gives the relevant literature reference; the second column provides the data types that are measured directly by the NIRS instrument used; the third column lists the parameter (usually derived from the measured data) considered as a surrogate of changes in CBF (an empty cell means that the measured data were directly correlated with MAP); the fourth column specifies the CA index, usually obtained from transfer function analysis (TFA, see Sec. 5.3) considering MAP as the input and the CBF index or NIRS data as the output; the fifth column lists the application, and the sixth column the number of subjects $(n)$; the last column reports a brief conclusion of the study, noting the level of agreement with TCD in the cases in which the study was done concurrently with TCD.

One important difference between the optical NIRS and DCS methods compared to TCD is that while diffuse optical methods are sensitive to the local cerebral microcirculation, TCD senses BF in large blood vessels, thus providing a more global measure of cerebral circulation. In fact CA is a complex phenomenon and it is not yet known whether monitoring CA on the basis of flow measurements in a large artery is reflective of the autoregulation behavior in the entire brain (including regions perfused by other large arteries) as well as at the microcirculation level. For example, one recent TCD study has shown that the CBF response following a thigh pressure cuff release maneuver is different in the MCA and in the ophthalmic artery. ${ }^{167}$ The importance of a regional monitor of CA is also stressed in a recent work based on NIRS methods on patients with carotid artery obstructions, where the changes in total hemoglobin concentration were taken as a surrogate of $\mathrm{CBF}$ changes, and correlated with changes in MAP. ${ }^{161}$ The authors concluded that spatial mapping of CA may be useful to discover regions that are more likely to develop hemodynamic infarction. ${ }^{161}$

We conclude this section with a brief comment on MAP measurements. As discussed previously, the parameter that affects $\mathrm{CBF}$ is the cerebral perfusion pressure $(\mathrm{CPP}=\mathrm{MAP}-\mathrm{ICP})$ and MAP can be used as a reliable surrogate of CPP only if ICP is negligible with respect to MAP. There are certain clinical conditions, such as severe head injury, in which this assumption is not valid. ${ }^{11}$ Common methods to measure MAP make use of an intravascular catheter, or a servo-controlled photoplethysmograph, as described in Sec. 4. The latter provides noninvasive, continuous beat-to-beat monitoring of ABP and MAP, and it is currently the method most largely used for studies on healthy subjects.

\subsection{Model-based methods}

Model-based methods for assessing CA rely on physiological and vascular models of cerebral hemodynamics with unknown parameters that are fitted to experimental data. Mathematical models of CA are usually devised to predict the behavior of $\mathrm{CBF}$ and other hemodynamic parameters on the basis of a conceptual characterization of blood vessels in terms of resistance and compliance as in fluid dynamic or electrical networks. The values of the physiological/vascular parameters retrieved by a fitting procedure provide valuable alternative metrics for CA to those provided by direct methods. Many models have been proposed to describe and characterize CA using a variety of conceptual frameworks: a flow-dependent feedback mechanism based on a time delay and a rate of regulation, ${ }^{168}$ a two-resistor and one-capacitor Windkessel model, ${ }^{169}$ an electrical circuit representation of a hydrodynamic model of the cerebrovascular flow that takes into account the effects of $\mathrm{ICP},{ }^{170}$ more complex electrical networks to model the variety of autoregulatory feedback mechanisms in the cerebral circulation, ${ }^{21,171-174}$ a multicompartment electrical equivalent network reflecting arterial, capillary, and venous hemodynamics, ${ }^{175}$ a physiologically based model that consolidates circulatory biophysics, metabolic biochemistry, and a treatment of vascular smooth muscle to describe brain circulation, energy metabolism, and related feedback pathways, ${ }^{176-178}$ etc.

In Sec. 5.2.1, we briefly describe the model of Ursino ${ }^{21}$ and the model of Payne and Tarassenko, ${ }^{175}$ which are representative of several models based on mass conservation principles and electrical analogs. In Sec. 5.2.2, we describe an alternative method for physiological parameter quantification and CA assessment, based on different principles, which was recently proposed by our group as a method of data analysis in CHS. ${ }^{127,131}$

\subsubsection{Two representative model-based methods for cerebral autoregulation}

The Ursino model ${ }^{21}$ was developed to describe the dynamics of intracranial vascular beds, the production and absorption of CSF and the dynamics of ICP. In this complex mathematical model, the intracranial dynamics are described by electrical analogs. More specifically, the system is divided into three parts, where the first two represent the precapillary vascular bed (proximal pial arteries and small arterioles) and the last one represents the capillary and postcapillary parts of the vascular bed. Each part is defined by its own specific conductance, compliance, ICP, and so on. The autoregulation mechanism is introduced in the two arterial compartments by means of two formally identical equations describing the tension of the vascular walls (due to muscular action) that depend, among other parameters, on one activation factor. The activation factor can be positive (when describing vasoconstriction) or negative (when describing vasodilation). The temporal dynamics of the activation factors in the two arterial compartments are introduced by two differential equations that depend on two key parameters of the CA mechanism: a time constant and a gain. The original model featured 21 equations and 40 free parameters. In the original study, ${ }^{21}$ the model was applied to simulate the ICP pulse wave. In a later study the model was also applied to simulate the dynamics of CBF, of the radius of proximal arteries and arterioles and also of ICP, to a step decrease in MAP. ${ }^{179}$ For these simulated data, the time constants describing the CA mechanism were fixed at $20 \mathrm{~s}$ (proximal arteries) and $10 \mathrm{~s}$ (arterioles). The model could reproduce typical observed behavior of CBF during the step decrease in MAP. The model has been used also for fitting experimental data, in particular the dynamic of ICP after saline injection in the craniospinal space, which is a typical test applied to very sick subjects. ${ }^{171}$ Of all the model parameters, only four were varied for reproducing the experimental data, including the gain and time constant of the autoregulation mechanism in the distal arterioles. The time constant retrieved by the fitting in eighteen subjects analyzed showed a wide individual variance and covered the range 2-380 s (average value $53 \mathrm{~s}$ and standard deviation $108 \mathrm{~s}$ ). 
A similar (yet more simplified) model, based on a flow dependent feedback mechanism was proposed by Payne and Tarassenko. ${ }^{175}$ The arterial compartment is divided into two sections, one characterized by a fixed resistance (reflecting the behavior of larger arteries) and the other characterized by a variable resistance and capacitance (reflecting the behavior of smaller arteries and arterioles). The capillary compartment is represented by a fixed resistance and capacitance, while the venous compartment is represented by two resistances, one lumped together with the capillary resistance, reflecting the behavior of smaller veins, and the other reflecting larger veins. The venous compartment is also characterized by a variable capacitance. In the model, only one (constant) value of ICP is assumed. The model uses differential equations for the dynamics of arterial and venous blood volumes, and the autoregulation mechanism is introduced by a state variable that has a role similar to the activation factor in the Ursino model. The state variable is used to adjust the arterial compliance and its dynamic behavior is specified by a temporal differential equation that (similarly to the Ursino model) depends on two parameters: the gain and the time constant of the autoregulation mechanism. In order to derive the impulse response functions (IRFs) of $\mathrm{CBF}$ and $\mathrm{CBFv}$ due to a change in MAP, the model equations are linearized around the baseline values. Strictly speaking, the model is used to derive the transfer functions (TFs) of the system, which are expressed in terms of the Laplace transforms of the IRFs. In particular the TF for CBFv depends on five parameters, including the time constant of the autoregulatory mechanism and another parameter that depends on the gain. The TF was fitted to experimental data by using only four (unknown) parameters and fixing the fifth parameter sequentially in several ranges, in order to study the sensitivity of the fitting procedure on that parameter.

\subsubsection{Cerebral autoregulation assessment with coherent hemodynamics spectroscopy}

As previously mentioned in Sec. 3.8.4, CHS is based on cerebral hemodynamic oscillations that are highly coherent with MAP oscillations induced by a forcing mechanism (e.g., paced breathing, cyclic thigh cuff inflation, etc.). When cerebral hemodynamic oscillations are properly induced, the cerebral concentrations of oxy- and deoxyhemoglobin become highly coherent and feature a stable phase and amplitude, which depend on the frequency of oscillation. The mathematical model ${ }^{127}$ provides a rather straightforward analytical relationship between CBF changes and the changes in oxy- and deoxyhemoglobin concentrations at different frequencies of the forcing mechanism (this is the frequency-domain version of the method). It also provides a relationship between CBF and oxy- and deoxyhemoglobin concentrations during transients and arbitrary temporal evolution of $\mathrm{CBF}$ (this is the time domain version of the method). The idea behind this relatively simple mathematical model is to treat the entire capillary and venous vascular compartments as low pass filters in a circulation system in which the input variables are the changes in blood volume, $\mathrm{BF}$, and oxygen consumption, and the output variables are the changes in oxy-, deoxy-, and total hemoglobin concentrations. By curve fitting the temporal trends of oxy- and deoxyhemoglobin changes (in either the time or frequency domain) we derive six physiological parameters. ${ }^{129,131}$ Among these parameters, we have already mentioned the capillary transit time and the capillary blood volume, which can be used to derive an absolute measure of CBF (see Sec. 3.8.4). Another important parameter derived with CHS is the autoregulation cutoff frequency, $f_{c}^{(A R)}$, which is a measure of the upper limit for the frequency of MAP oscillations that can be regulated by CA. In fact, in the literature, the CA process has been often modeled as a high pass filter having MAP as input and CBF as output. In our mathematical model, the CA process is described by a resistor-capacitor (RC) high pass filter, with normalized CBV changes as input and normalized CBF changes as output. The filter is characterized by a cutoff frequency, which is usually in the order 0.01$0.10 \mathrm{~Hz}$. The larger this parameter, the more efficient the CA process is.

A validation test performed on healthy human subjects during hyperventilation-induced hypocapnia has shown that the autoregulation cutoff frequency is significantly greater during hypocapnia $(0.034 \pm 0.005 \mathrm{~Hz})$ with respect to normocapnia $(0.017 \pm 0.002 \mathrm{~Hz}) .{ }^{166}$ This result of enhanced autoregulation during hypocapnia is in agreement with studies based on different methods of grading CA. ${ }^{19}$ We place this study in Table 3 although we note that this study was model-based, while the other NIRS studies in the table were based on direct methods and data analysis, as described in Secs. 5.1 and 5.3.

Other hemodynamic models based on sets of unknown physiological parameters have been proposed for the interpretation of NIRS data. However, these models do not address directly the problem of estimating a metric for CA, and most of them are based on complex systems of partial differential equations that make them computationally intensive (for a list of these models we refer to Ref. 180).

\subsection{Methods of data analysis for cerebral autoregulation}

In this section, we review some methods of data analysis for CA that are used with the direct methods discussed in Sec. 5.1. These methods are based on the adoption of a signal processing perspective. In other words, these methods do not attempt to understand the phenomenon of CA from first principles, but they rather try to describe the relationship between MAP and $\mathrm{CBF}$ in a number of different conditions. One of the goals is to find an effective metric for $\mathrm{CA}$ assessment that may be used in clinical settings. Even if not exhaustive, this section summarizes some of the most popular methods that have been used for data analysis of CA (mostly in TCD studies).

\subsubsection{Methods for static cerebral autoregulation}

For data analysis of static CA, essentially three methods (with some variations) have been adopted:

(a) Linear regression analysis between CBF (usually relative to its baseline values) and MAP;

(b) Pearson correlation analysis;

(c) Monitoring of cerebrovascular reactivity (CVR) as MAP is changed.

The last method has been used mainly in those studies in which MAP (and therefore CBF) was changed only one time with respect to its baseline values. These methods are rather straightforward and they are usable to define CA either in a binary way (i.e., intact or impaired), or to define different levels of CA. 
In method (a), a threshold slope was defined, usually in the range $0.5-3-4 \% \mathrm{CBF} / \mathrm{mmHg}$, for the binary classification, so that any slope greater than the threshold slope was considered indicative of impaired CA. ${ }^{11}$ In other studies, a multiple linear regression was applied with $\mathrm{CBF}$ as dependent variable and MAP, partial pressure of oxygen $\left(\mathrm{pO}_{2}\right)$ and partial pressure of carbon dioxide $\left(\mathrm{pCO}_{2}\right)$ as regressors. In fact, it is well known that $\mathrm{CBF}$ is affected independently by other covariates of MAP. In static methods of CA assessment that need longer measurement times, the effect of these regressors on $\mathrm{CBF}$ changes is more prominent than for dynamic CA methods (for a list of these studies, we refer to ${ }^{11}$ ).

According to method (b), a Pearson correlation coefficient (r) can similarly be used to discriminate between intact (low correlation) and impaired (high correlation) CA. The reasoning is that if $\mathrm{CA}$ is impaired $\mathrm{CBF}$ changes are more passive to MAP changes, and a high value of the correlation coefficient is expected. In some studies $r=0.5$ was chosen as a threshold to discriminate between the two CA conditions. ${ }^{181}$

In method (c), only two values of MAP were used, and an index of static CA could be defined based on the slope of changes in CVR. This index has been variably named SARI (static autoregulation index) $^{11}$ or SCA (static CA) index. ${ }^{182}$ Despite different names, the two indices share the same definition: $\mathrm{SCA}=\mathrm{SARI}=\% \mathrm{CVR} / \% \mathrm{MAP}$, where $\% \mathrm{CVR}$ is the change in CVR (in percent of its baseline value) and \%MAP is the change in MAP (expressed also in percent of its baseline value). We remind that if $\mathrm{CPP} \cong \mathrm{MAP}, \mathrm{CVR}$ is defined by

$\mathrm{CVR}=\frac{\mathrm{MAP}}{\mathrm{CBF}}$

By differentiating Eq. (31), we obtain

$$
\frac{\Delta \mathrm{CVR}}{\mathrm{CVR}}=\frac{\Delta \mathrm{MAP}}{\mathrm{MAP}}-\frac{\Delta \mathrm{CBF}}{\mathrm{CBF}} .
$$

It is clear from Eq. (32) that if CBF follows MAP passively, $\Delta \mathrm{CVR} / \mathrm{CVR}=0$ and $\mathrm{SARI}=0$. On the contrary, if the change of CVR fully compensates the change in MAP (i.e., $\frac{\Delta C V R}{C V R}=\frac{M A P}{M A P}$, there is no change in $\mathrm{CBF}$ and SARI $=1$. This method of grading static CA has been used to compare static and dynamic CA indices. ${ }^{182}$ Recently, a more complex method of nonlinear regression (the project pursuit regression), has been used to define the static MAP-CBF curve. ${ }^{28}$ The static curve MAP-CBF was measured by using an oscillatory lower body negative pressure (OLBNP) maneuver. For each frequency of oscillation, the lower body negative pressure was changed in a given range and the corresponding average MAP and CBF amplitudes of oscillation were measured and plotted against each other. The authors were able to demonstrate that at lower frequency of oscillations $(0.03 \mathrm{~Hz})$, the MAP-CBF curve follows the usual characteristic autoregulation curve, with a central region of MAP values where changes of MAP were adequately buffered (region of low slope and efficient CA). However, at the highest frequency of oscillation $(0.08 \mathrm{~Hz})$, the effectiveness of CA was dramatically reduced, and the region where CA was more efficient had a steeper slope. The motivation behind the adoption of a nonlinear model for describing the relationship between MAP and CBF is that it is well known that at lower oscillation frequencies, pressure and flow become less linearly related and their mutual coherence becomes not significant, indicating that the phenomenon of $\mathrm{CA}$ is nonlinear. ${ }^{28}$

\subsubsection{Methods for dynamic cerebral autoregulation}

The methods for assessment of dynamic CA have been devised mainly to take advantage of the continuous monitoring of MAP and CBF (as, for example, with TCD). With these methods, the definition of different levels of CA has become more straightforward than for static methods. Most of the methods assume that the input-output system MAP-CBF (where MAP is the input and CBF is the output) is linear and time invariant. This can be a reasonable assumption for small changes of MAP and CBF, as for example during baseline data, but it should be tested for maneuvers in which MAP changes by relatively large amounts (e.g., thigh cuff release method). These methods have been developed both in the time domain and frequency domain.

In the frequency domain, one test of linearity between two signals is given by the coherence function. High values of the coherence function indicate a linear relationship between two signals. On the contrary, a low coherence value can be interpreted in several ways as being indicative of: (1) a nonlinear relationship, (2) a poor signal-to-noise ratio (SNR), (3) a lack of relationship between the two signals, or (4) a multiple inputs-single output relationship. ${ }^{183}$ Frequency domain methods have been applied to describe phase and amplitude relationships between MAP and CBF either during baseline data or during induced MAP oscillations at a single or multiple frequencies. In the time domain, most of the methods are used to describe the transient of MAP and $\mathrm{CBF}$ during a thigh cuff release maneuver.

One simple method for differentiating different levels of CA was introduced by Aaslid et al. ${ }^{19}$ during a thigh cuff release maneuver. The method takes advantage of the continuous monitoring of MAP and CBF by defining a time-dependent CVR. The authors demonstrated that in the first few seconds after the cuff release, CVR showed a linear trend as a function of time, in which the slope was sensitive to the level of CA. We note that an ideally working CA mechanism would instantly compensate for the change of MAP, and CVR as a function of time would be a vertical line (infinite slope). However, since the negative feedback loop that is rebalancing CBF through the adjustment of CVR (at least according to the metabolic theory of CA), needs a finite operation time, the slope is also finite. Similarly to the static SARI or SCA indices, the authors defined a rate of autoregulation $\mathrm{RoR}=$ $\% \mathrm{CVR} / \% \mathrm{MAP} / \Delta t$, where $\Delta t$ is the time interval where the linear trend of CVR can be assumed. The authors found different RoRs according to the level of $\mathrm{CO}_{2}$ present in the blood, with the steepest slope during hypocapnia, and the shallower slope during hypercapnia.

The same group proposed a more analytical model of CA based on a state space model of the ordinary differential equation (ODE) governing the temporal relationship between MAP and CBF during an ideal step decrease of MAP. ${ }^{182}$ Usually, the state variables in a state space model depend on the order of ODE, and for the case of the method proposed by Tiecks et al., the authors chose two state variables. ${ }^{182}$ The model depends also on three parameters: a damping factor $(D)$, an autoregulation gain $(K)$ and a time constant $(T)$. Arbitrarily, the authors chose ten different triplets for the three parameters and associated one single index (the autoregulation index, ARI) 
to describe the level of autoregulation. For each ARI, the state space model is solved and a CBF curve as a function of time is calculated for the transient after a thigh cuff release. An ARI $=$ 0 corresponds to no $\mathrm{CBF}$ response (which follows passively $\mathrm{MAP}$ ), and an ARI $=9$ is characterized by the fastest recovery time for CBF to its baseline value. This "library" of solutions for $\mathrm{CBF}$ after thigh cuff release is used for defining the ARI of a measured $\mathrm{CBF}$ curve as a function of time by least square fitting.

Instead of studying transients of MAP and CBF during a sudden change of $\mathrm{ABP}$, like in the thigh cuff maneuver, other researchers have induced hemodynamic oscillations at a single ${ }^{161,184,185}$ or multiple frequencies ${ }^{186}$ and studied the phase, amplitude, and coherence relationships between MAP and CBF. Typical maneuvers adopted to induce oscillations are paced breathing, ${ }^{161,184}$ squatting, ${ }^{185}$ or lower body negative pressure. ${ }^{28}$ The systematic study of phase, gain and coherence between MAP (input) and CBF (output) at multiple frequencies (ideally a continuous set of frequencies) is called TFA. Here we note that time and frequency domain analysis (or TFA) of CA are related by a Fourier transformation, and while the former aims at extracting the IRF, the latter aims at extracting the TF between MAP (input) and CBF (output). Pioneering studies using TFA date back to the early 1990 s, ${ }^{187}$ but here we summarize the results of the study carried out by Zhang et al., ${ }^{183}$ which have been reproduced in multiple independent studies.

The interesting aspect of the study by Zhang et al. is that TFA was applied to 6 min of rest data taken on healthy subjects, and TFA was applied to estimate the TF of the system MAP (input) $\mathrm{CBF}$ (output). ${ }^{183}$ The TF was estimated by calculating the ratio of the cross spectrum between MAP and CBF, and the auto spectrum of MAP. The coherence function was estimated by the ratio of the absolute value of the cross spectrum between MAP and CBF squared and the product of the autospectrum of MAP and CBF. The group average carried out on ten subjects showed that the amplitude of TF (also called gain) increased in the range of 0.07 to $0.2 \mathrm{~Hz}$, and that the coherence was larger than 0.5 for frequencies larger than $0.3 \mathrm{~Hz}$. The phase of the TF decreased in the range of 0.07 to $0.5 \mathrm{~Hz}$ from about 1 radian to 0 . These results are typical of a high-pass filter relationship between MAP and CBF, at least in the range where coherence was significant. The authors also discussed the possible interpretations of low coherence values. The IRF, calculated by an inverse Fourier transform of the TF, agreed well with the IRF measured during a thigh cuff release. Strictly speaking, with this maneuver one can measure the step response function; however, this function is related to the IRF by a simple time derivative. Measurements of IRF and TF from baseline data would be ideal for CA studies, since the influence of covariates of MAP changes on $\mathrm{CBF}$ changes are minimal. However, the main challenge of using spontaneous oscillations of MAP and CBF is to achieve a significant SNR, or, in other words, to select those spontaneous fluctuations of CBF that are not due to random noise but are caused by similar fluctuations of MAP. For this reason, Panerai et al. proposed the method of coherent averaging ${ }^{188,189}$ before applying TFA analysis. This method consists of finding a certain number of MAP fluctuations (about 30 in their original studies) where the peak-to-peak change of MAP is larger than $2 \%$ of the baseline value. The coherent averaging of MAP fluctuations and of the corresponding $\mathrm{CBF}$ oscillations is done by defining a point of synchronism, which is the point of maximum derivative in the rising part of each MAP oscillation.
Other models of data analysis that assume a linear relationship between MAP and CBF are the autoregressive moving average processes (ARMA). An ARMA model is a process in which a system is described by a single input-single output relationship (as for MAP and CBF in CA) and the output at a certain time is obtained as a linear combination of output values at previous times and of input values at previous and current times. We can say that an ARMA model is applied to stochastic data in an attempt to find the law governing the relationship between input and output. The mathematical form of an ARMA model resembles a discretized differential equation, with the difference that in this case the coefficients must be determined from the data and are not given a priori. Usually, one aims to find the ARMA model with the least number of coefficients that can reliably reproduce the data. A comparison of different ARMA models was performed to study the relationship between MAP and CBF in neonates. ${ }^{190}$

Nonlinear models have also been used to describe CA. An interesting comparison between linear and nonlinear models is reported in Ref. 191. Measurements of MAP and CBF were recorded on 47 healthy subjects during two periods of rest (5 min each) and during a sudden thigh cuff deflation protocol. Different methods of predicting the IRF of the CBF response were used: (a) TFA, (b) Tiecks model with ARI, and (c) the method of Volterra-Wiener kernels by considering either the linear term, or both the linear term and the quadratic term. The IRFs were obtained by the different methods during one of the two rest periods (training dataset) and were then tested on the other rest period and during the thigh cuff release maneuver (test datasets) to reproduce the CBF temporal trends. This was done by using the convolution of the IRFs with MAP temporal trends. During the training data set, the correlation between the measured and predicted CBF temporal trends was significant for all the models, but the Volterra kernel method with the quadratic term yielded the highest correlation. However, when the same IRFs were applied to the other rest period and to the thigh cuff release maneuver (test data set) to reproduce the CBF temporal trend, the linear models yielded similar performances that were comparable to those obtained for the training dataset, while the nonlinear model yielded the poorest correlation. The authors explained this result by the lack of randomness in the input data (MAP spontaneous oscillations), which is required by the Volterra-Wiener method and that makes the method too sensitive to the training dataset.

Finally, other methods have released the assumption of linearity and/or stationarity between MAP and CBF (implicit in the TFA method), and require more complex mathematical constructs like Volterra kernels, ${ }^{191,192}$ continuous complex wavelet, ${ }^{193}$ the Hilbert transform, and empirical mode decomposition. ${ }^{194}$

\section{Cerebral perfusion assessments in the clinical practice}

The brain is a highly metabolically active organ that places a high demand on the body for nutrients and resources. The utility of measurement of CBF and CA in clinical practice can be divided into two realms: investigational use - of which there is a plethora, and practical clinical use - of which there is less robust but no less interesting data. Static measurements of CBF and CA are currently used in the diagnosis and assessment of disease states, while dynamic resting state or task oriented assessment is useful in surveillance of disease fluctuation or response to 
therapy. Of clinically relevant metrics of $\mathrm{CBF}$ and CA it is helpful to approach two different aspects of clinical careinvestigation of chronic diseases and investigation and management of acute illness, which will be discussed below. This is not meant to be an exhaustive review of the clinical utility of $\mathrm{CBF}$ and $\mathrm{CA}$ assessment but rather to highlight some practical applications.

\subsection{Chronic central nervous system diseases- understanding pathobiology and treatments}

Certain neurodegenerative diseases have been associated with changes in regional or global CBF. Cerebral hypoperfusion and vascular dysfunction are thought to be related to the pathogenesis of Alzheimer's disease (AD) thus implicating that the measurement of CBF might be a useful biomarker of disease incidence or progression. ${ }^{195}$ Though mostly investigational, MRI, ${ }^{196}$ SPECT, ${ }^{197}$ and NIRS ${ }^{198}$ are rapidly approaching prime time to be a useful marker of $A D$ via a point estimate of CBF. Noninvasive assessment of dynamic CBF has also recently shed some light on how certain medications used to treat dementia, notably donepezil ${ }^{199}$ and memantine, ${ }^{198}$ exert their cognition-preserving effects by perhaps protecting $\mathrm{CBF}$ integrity over time. Analogous to these studies are $\mathrm{CBF}$ assessments in Parkinson's disease (PD). Single point in time measurements of regional cerebral hypoperfusion have been shown to correlate with certain symptoms of PD along with a corresponding increase in $\mathrm{CBF}$ after treatment with both pharmacologic agents ${ }^{200}$ and functional neurosurgical interventions. ${ }^{201}$ Assessment of CA is also of interest in these diseases. Broadening of the cerebral autoregulatory maintenance threshold has been shown as a potentially adaptive mechanism to orthostatic hypotension and supine hypertension that is common in PD and multiple system atrophy, and is noticeably contrary to animal models. ${ }^{202}$ Some degree of the breakdown of $\mathrm{CA}$ is associated with normal aging, but there seems to be an additional association between reduced CA, amyloid plaque deposition, and white matter disease. ${ }^{203}$ This potentially conflicting information is evidence that the exquisite nature of chronic cerebral pathology, $\mathrm{CBF}$ and $\mathrm{CA}$ is still in its infancy of being understood and more work is needed.

Alterations in CBF have been of academic interest in psychiatric illnesses since the 1940s. ${ }^{204}$ Advances in technology have allowed a renewed interest in the pathophysiology and clinical biomarkers of these diseases. PET, SPECT and MR perfusion, and fMRI-based global and regional CBF methods have resulted in new insights into these debilitating chronic disorders. ${ }^{205}$ Both dynamic task-related assessments and resting state assessments have been invaluable in characterizing the neurobiologic correlates of symptoms in schizophrenia. Both regional cerebral hypoperfusion and hyperperfusion have been associated with various positive and negative symptoms in schizophrenia. ${ }^{206}$ Assessment of CBF has also revealed alterations in regional brain perfusion in bipolar disorder ${ }^{207}$ and major depressive disorder. $^{208}$ Dynamic changes in regional CBF using fNIRS and fMRI have been used to assess neurobiologic correlates of these diseases as well as attention deficit disorder. ${ }^{209}$ Though academically intriguing, both snapshot assessments of CBF and dynamic responses to therapy are not widely used to guide clinical therapy.

Static CBF and tissue perfusion assessment are quickly becoming a useful part of the workup of brain neoplasms, specifically cerebral gliomas. Understanding the histopathological grade of these tumors is useful for diagnosis and prognosis as well as guiding surgical, chemotherapeutic and radiation therapies. Various MR and CT based techniques have been used to determine a relative $\mathrm{CBF}$ ratio, in addition to a relative $\mathrm{CBV}$ ratio, and can be a useful tool in the evaluation of grading of cerebral gliomas prior to surgery. ${ }^{210-212}$

Ischemic stroke, arguably the most severe manifestation of acute reduction in regional $\mathrm{CBF}$, is often managed with carotid endarterectomy for secondary stroke prevention due to critical carotid artery stenosis. Most complications from this procedure are ischemic, either from intraoperative embolus or inadequate intraoperative brain perfusion. One feared complication is the postoperative hyperperfusion syndrome, which can lead to cerebral edema and intracerebral hemorrhage in the most severe cases. ${ }^{213}$ Efforts to predict who would be at risk for this complication and to guide postoperative perfusion thresholds have led some vascular surgeons to assess CA, CVR, and CBF via single-photon emission $\mathrm{CT}$ with acetazolamide challenge and internal carotid artery flow measurements during surgery. ${ }^{214}$ Reduced CVR preoperatively has been shown to positively correlate with postoperative hyperperfusion allowing for risk stratification of the patients for complications in the postoperative period and perhaps allowing more individualized treatment. ${ }^{215}$

Intraoperative measurement of real-time $\mathrm{CBF}$ and CA during neurovascular procedures has become a routine practice for some surgeons. ${ }^{216}$ Various methods ranging from contrastaided video angiography to more novel laser speckle contrast analysis have allowed neurosurgeons to have a dynamic assessment of CBF during surgeries ranging from aneurysm repair and reconstruction, cerebral arterial bypass surgery, and operative treatment of cerebral atherosclerotic disease. ${ }^{216,217}$

\subsection{Acute central nervous system diseases- assessment and treatment}

Perhaps the most useful and exciting application of both realtime and static measurements of $\mathrm{CBF}$ and $\mathrm{CA}$ is in the care of the patient with acute central nervous system injury. In the care of the patient with severe brain injury from a variety of mechanisms, notably TBI, aneurysmal subarachnoid hemorrhage (SAH) and acute ischemic stroke (AIS), supporting the injured brain with adequate $\mathrm{CBF}$ and delivery of oxygen and energy substrate is the main goal in the acute care. Basic management of these patients requires calculation of a CPP as a surrogate for global CBF. Recent evidence suggests that monitoring ICP, and therefore CPP, alone may not significantly alter functional outcomes after TBI, and opens the door for a more individualized plan of care. ${ }^{218}$ Intracranial hypertension and calculated CPP is likely not a sensitive indicator of cerebral hypoxic/ischemic injury. This method doesn't take into account the multicompartmental nature of heterogeneous brain injuries. Ischemic brain injury occurs in the acute stage of over $80 \%$ of severe TBIs and is common in other nontraumatic brain lesions. $^{219}$ Individualizing care has led to consensus guidelines incorporating $\mathrm{CA}$-recent guidelines by the Neuro-Intensive Care and Emergency Medicine (NICEM) section of the European Society of Intensive Care Medicine specifically address this and suggest that $\mathrm{CPP}$ targets $>70 \mathrm{mmHg}$ in braininjured patients should be targeted only if $\mathrm{CA}$ is determined to be intact. $^{220}$

Monitoring of $\mathrm{CBF}$ and $\mathrm{CA}$ has applications in the research aspect of the evolving field of neurocritical care. Osmotic agents such as mannitol and hypertonic saline (HTS) are standard of 
care for patients with cerebral edema, intracranial hypertension and cerebral herniation syndromes. Recent evidence using PET shows that in addition to lowering ICP and reversing herniation syndromes, osmotic therapy can improve regional $\mathrm{CBF}$ as well. ${ }^{221}$ This investigation found that administration of mannitol or HTS increased CBF specifically in regions with baseline hypoperfusion without increasing global CBF.

Autoregulatory failure and cerebral hyperperfusion are less common acute illnesses. The prototypic diseases associated with arterial hypertension and dysregulation of CA are the posterior reversible encephalopathy syndrome and eclampsia. ${ }^{222}$ Conflicting data regarding the exact role of $\mathrm{CA}, \mathrm{CBF}$ thresholds, and pre-existing autoregulatory curves make these diseases still poorly understood and more work needs to be done to understand their pathophysiology. ${ }^{223}$ The role of assessing CBF and $\mathrm{CA}$ in real time to guide treatment is also poorly understood at this time.

Delayed cerebral ischemia (DCI) is one of the most feared complications of SAH. Perhaps the simplest, cheapest, and most widely used index of CBF in these patients is TCD. TCD has the benefit of being able to detect cerebral vasospasm and give real-time bedside indicators of $\mathrm{CA}^{224}$ Bedside TCD assessment of $\mathrm{CBF}$ velocities and $\mathrm{CA}$ has shown to be highly predictive of the incidence of DCI and may further augment individualized care of these patients. ${ }^{225}$ Static metrics of whole brain CBF, CBV, and MTT using perfusion CT are used routinely to assess cerebral vasospasm and DCI but can also help to risk-stratify those who may develop DCI during the course of their illness. ${ }^{226}$ This modality can be limited clinically by the risks of intravenous contrast agents and multiple exposures to radiation. Though proven as feasible, continuous TCD measurement of response to therapy in patients with cerebral vasospasm has proven cumbersome to do at the bedside and is not routinely used in practice. ${ }^{227}$ Recently, fMRI has been postulated as a useful tool to identify brain at risk for DCI after SAH by assessing CVR. ${ }^{228}$ While an exciting technology, critically ill patients are often not able to participate with MRI scanning potentially limiting the utility. Assessment of CA after SAH is available via multiple methods. In one study, TCDderived and NIRS-derived metrics were superior to transient hyperemic response ratio, though an index comprised of all three was $100 \%$ sensitive in predicting the development of DCI. ${ }^{229}$ A promising advent in the ongoing care of these patients is the advance in NIRS-based metrics of CBF and CA. Recent studies have shown that continuous real-time measurement of tissue oxygenation index is a feasible approach at the bedside for these patients ${ }^{159}$ and can identify thresholds of cerebral ischemia allowing the clinician to individualize CPP targets of individual patients and for the duration of the patients' illness. $^{230}$

Another devastating yet heterogeneous illness is TBI. TBI is one of the leading causes of death and disability in young adults. The goal of caring for patients with severe TBI is attenuation of secondary injury that occurs minutes to days after the primary injury and is focused on maintenance of adequate cerebral perfusion and oxygenation. ${ }^{231} \mathrm{CA}$ has been shown to be disrupted in up to one third of patients with severe TBI, ${ }^{232,233}$ making the injured brain "pressure passive" and highly susceptible to ongoing ischemic injury. TBI is not a single disease and the best way to care for these patients as a group remains an elusive target. TBI patients with diffuse injuries likely have different CPP and CBF targets than those with mass lesions and should be treated as such. ${ }^{234}$ ICP monitoring for severe TBI patients remains the standard of care and is currently recommended by the Brain Trauma Foundation. ${ }^{231}$ Currently, the best technology available to do this and to be able to calculate CPP relies on surgically implanted probes. Current technology to measure real-time dynamic CBF is somewhat limited to TDF and $\mathrm{LDF}$, both invasive tools requiring an implantable probe. ${ }^{235}$ These are useful, but have failed to guide widespread use due to acquisition cost of the technology, the limited information from tissue directly adjacent to the probe, and the invasive nature of the monitors and inherent risks from implantation. Diffuse optical imaging and NIRS have been validated as providing comparable data to LDF in TBI in regards to total hemoglobin, oxyhemoglobin and deoxyhemoglobin concentrations, ${ }^{236}$ and also are comparable to perfusion CT for regional CBF monitoring in brain injured patients. ${ }^{237}$ Additional realtime regional $\mathrm{CBF}$ monitoring is available via brain surface TDF but also is invasive and used mostly in experimental application and has not gained widespread acceptance. ${ }^{238}$

\section{Clinical prospects of noninvasive optical measurements of blood flow and autoregulation}

$\mathrm{CBF}$ and CA assessment is used widely in research but the transition to well accepted and standardized approaches in clinical care has been slow. Primary limitations are the access to technology that might not be available to all practitioners, cost, and the invasive and time consuming nature of various technologies. An ideal technology measurement of $\mathrm{CBF}$ and autoregulation would be inexpensive, easy to apply, noninvasive, harmless, have a high inter-rater reliability, and allow for real-time assessment of pathology as well as dynamic changes based on evolving illness and response to therapy.

TCD fulfills many of these criteria but TCD measurements are significantly operator-dependent and can have a wide interexaminer variability. ${ }^{239}$ Transcranial color-coded sonography has the advantage of visualization of tissues and reducing sampling error but comes with increased cost and training of the sonographer. ${ }^{240}$ TCD monitoring of CA and CVR can also be affected by patient position making day-to-day trending of these variables potentially troublesome. ${ }^{241}$ Automated TCD monitoring of CBF through the MCA are available to reduce inter-rater variability as with the validated Presto 1000 TCD system (PhysioSonics, Bellevue, WA), ${ }^{242}$ but this technology is in its infancy and gives limited clinical information.

Available implantable monitors utilizing LDF or TDF suffer from all the limitations of any invasive procedure leaving the vulnerable patient at risk for additional pain, infection, bleeding, and the potential for dislodging probes during the routine care of the patient. This, in addition to lack of validation studies supporting that their use alters outcomes after brain injury, has led to their use being mostly experimental. ${ }^{238}$

MRI and CT hardware are relatively common in modern healthcare, making both MR-based and CT-based assessment of $\mathrm{CBF}$ and CA potentially available to a wide population. Both healthy subjects and critically ill patients may have significant contraindications to either or both of these, making the standardization of application to all patients difficult. Most CT-based tools use intravenous contrast agents that some patients cannot have either due to allergy or renal dysfunction. CT also requires significant radiation exposure. Recent data refutes the notion that repeated exposures in TBI patients 
alter long-term cancer risk, but clinicians should try to minimize radiation exposure whenever possible. ${ }^{243}$ MR technology can be severely limited by cost. Acquisition cost for an MR can be in the millions of dollars, and a single MRI can cost payers thousands of dollars. ${ }^{244}$ MR technology is also limited by the inherent dangers of strong magnets making assessment of CBF and CA in critically ill patients logistically very difficult and potentially dangerous.

NIRS is a very attractive technology fulfilling nearly all the gaps in current technology to assess both a reasonable point estimate of $\mathrm{CBF}$ and $\mathrm{CA}$ as well as potentially offer real-time regional $\mathrm{CBF}$ information to the clinician. NIRS has been validated as a reliable bedside tool for dynamic measurement of regional $\mathrm{CBF}$ in patients with $\mathrm{SAH}$, ischemic stroke and intracerebral hemorrhage ${ }^{237}$ and TBI. ${ }^{245}$ Studies using NIRS to evaluate routine aspects of care for brain injured patients have shed light on the effect of maneuvers such as head of bed angle manipulation on $\mathrm{CBF}$, further setting the stage for prospective trials to validate the utility of this exciting technology going forward. ${ }^{245}$ Recently, wireless NIRS devices to detect brain hemoglobin levels have been validated in animals ${ }^{246}$ and will hopefully be validated as a tool to measure CBF in humans soon. A simple, harmless, wireless device to provide real-time regional $\mathrm{CBF}$ of the brain-injured patient has the potential to dramatically change the way we care for these patients. Additional studies still need to be performed to assess the impact on outcomes after brain injury however.

\section{Conclusions}

In summary, this comprehensive review of the state of the art of $\mathrm{CBF}$ and $\mathrm{CA}$ assessment in the research and clinical arenas shows that optical techniques have a significant potential for practical measurements of cerebral perfusion. In fact, they are safe, noninvasive (or minimally invasive in the case of intravenous administration of contrast agents), applicable at the bedside, and suitable for quantitative measurements and continuous monitoring. Advances in real-time measurements of $\mathrm{CBF}$ and $\mathrm{CA}$ have the potential to significantly improve the understanding of a variety of neurological conditions and alter outcomes after devastating brain injuries.

\section{Acknowledgments}

We acknowledge support from the National Institutes of Health (Grant R01-CA154774).

\section{References}

1. M. Bélanger, I. Allaman, and P. J. Magistretti, "Brain energy metabolism: focus on astrocyte-neuron metabolic cooperation," Cell Metab. 14, 724-738 (2011).

2. M. S. Vavilala, L. A. Lee, and A. M. Lam, "Cerebral blood flow and vascular physiology," Anesthesiol. Clin. N. Am. 20, 247-264 (2002).

3. Y. C. Tzeng and P. N. Ainslie, "Blood pressure regulation IX: cerebral autoregulation under blood pressure challenges," Eur. J. Appl. Physiol. 114, 545-559 (2014).

4. T. W. Barber, J. A. Brockway, and L. S. Higgins, "The density of tissue in and about the head," Acta. Neurol. Scandinav. 46, 85-92 (1970).

5. N. A. Lassen, "Normal average value of cerebral blood flow in younger adults is $50 \mathrm{ml} / 100 \mathrm{~g} / \mathrm{min}, "$ J. Cereb. Blood Flow Metab. 5, 347-349 (1985).

6. H. Girouard and C. Iadecola, "Neurovascular coupling in the normal brain and in hypertension, stroke, and Alzheimer disease," J. Appl. Physiol. 100, 328-335 (2006).

7. E. Bor-Seng-Shu et al., "Cerebral hemodynamics: concepts of clinical importance," Arq. Neuropsiquiatr. 70, 357-365 (2012).
8. R. C. Koehler, R. J. Roman, and D. R. Harder, "Astrocytes and the regulation of cerebral blood flow," Trends Neurosci. 32, 160-169 (2009).

9. S. J. Payne, "A model of the interaction between autoregulation and neural activation in the brain," Math. Biosci. 204, 260-281 (2006).

10. C. O. Tan and J. A. Taylor, "Integrative physiological and computational approaches to understand autonomic control of cerebral autoregulation," Exp. Physiol. 99, 3-15 (2014).

11. R. B. Panerai, "Assessment of cerebral pressure autoregulation in humans-a review of measurement methods," Physiol. Meas. 19, 305338 (1998).

12. H. J. Knot and M. T. Nelson, "Regulation of arterial diameter and wall $\left[\mathrm{Ca}^{2+}\right]$ in cerebral arteries of rat by membrane potential and intravascular pressure," J. Physiol. 508, 199-209 (1998).

13. H. A. Kontos, "Regulation of the cerebral circulation," Ann. Rev. Physiol. 43, 397-407 (1981).

14. C. Iadecola, "Neurovascular regulation in the normal brain and in Alzheimer's disease," Nat. Rev. Neurosci. 5, 347-360 (2004).

15. V. Pollard et al., "Cerebral blood flow during experimental endotoxemia in volunteers," Crit. Care Med. 25, 1700-1706 (1997).

16. J. Wolff et al., "Effects of eltanolone on cerebral blood flow and metabolism in healthy volunteers," Anesthesiology 81, 623-627 (1994).

17. D. R. Jobes et al., "Effects of morphine-nitrous oxide anesthesia on cerebral autoregulation," Anesthesiology 42, 30-34 (1975).

18. T. Numan et al., "Static autoregulation in humans: a review and reanalysis," Med. Eng. Phys. 36, 1487-1495 (2014).

19. R. Aaslid et al., "Cerebral autoregulation dynamics in humans," Stroke 20, 45-52 (1989).

20. N. A. Lassen, "Cerebral blood flow and oxygen consumption in man," Physiol. Rev. 39, 183-238 (1959).

21. M. Ursino, "A mathematical study of human intracranial hydrodynamics Part I- the cerebrospinal fluid pulse pressure," Ann. Biomed. Eng. 16, 379-401 (1988).

22. S. J. Payne, J. Mohammad, and M. M. Tisdall, "Effects of arterial blood gas levels on cerebral blood flow and oxygen transport," Biom. Opt. Exp. 2, 966-979 (2011).

23. B. Schmidt et al., "Cerebral autoregulatory response depends on the direction of change in perfusion pressure," J. Neurotrauma 26, 651656 (2009).

24. R. Aaslid et al., "Asymmetric dynamic cerebral autoregulatory response to cyclic stimuli," Stroke 38, 1465-1469 (2007).

25. M. Fog, "Cerebral circulation. II. Reaction of pial arteries to increase in blood pressure," Arch. Neurol. Psychiatry 41, 260-268 (1939).

26. C. K. Willie et al., "Regional brain blood flow in man during acute changes in arterial blood gases" J. Physiol. 590, 3261-3275 (2012).

27. N. E. Dineen et al., "Continuous estimates of dynamic cerebral autoregulation during transient hypocapnia and hypercapnia," J. Appl. Physiol. 108, 604-613 (2010).

28. C. O. Tan, "Defining the characteristic relationship between arterial pressure and cerebral flow," J. Appl. Physiol. 113, 1194-1200 (2012).

29. P. Meier and K. L. Zierler, "On the theory of the indicator-dilution method for measurement of blood flow and volume," J. Appl. Physiol. 6, 731-744 (1954).

30. K. L. Zierler, "Theoretical basis of indicator-dilution methods for measuring flow and volume," Circ. Res. 10, 393-407 (1962).

31. K. Høedt-Rasmussen, E. Sveinsdottir, and N. A. Lassen, "Regional cerebral blood flow in man determined by intra-arterial injection of radioactive inert gas," Circ. Res. 18, 237-247 (1966).

32. S. S. Kety, "The theory and applications of the exchange of inert gases at the lungs and tissues," Pharmacol Rev. 3, 1-41 (1951).

33. P. Herscovitch and M. E. Raichle, "What is the correct value for the brain-blood partition coefficient for water?" J. Cereb. Blood Flow Metab. 5, 65-69 (1985).

34. S. S. Kety and C. F. Schmidt, "The determination of cerebral blood flow in man by the use of nitrous oxide in low concentrations," Am. J. Physiol. 143, 53-66 (1945).

35. R. J. Traystman, "The paper that completely altered our thinking about cerebral blood flow measurements," J. Appl. Physiol. 97, 1601-1602 (2004).

36. S. S. Kety and C. F. Schmidt, "The nitrous oxide method for the quantitative determination of cerebral blood flow in man: theory, procedure and normal values," J. Clin. Invest. 27, 476-483 (1948). 
37. E. M. Wilson and J. H. Halsey, "Bilateral jugular venous blood flow by thermal dilution," Stroke 1, 348-355 (1970).

38. C. Mélot et al., "Estimation of cerebral blood flow at bedside by continuous jugular thermodiluation," J. Cereb. Blood Flow Metab. 16, 1263-1270 (1996).

39. D. H. Ingvar and N. A. Lassen, "Quantitative determination of regional cerebral blood flow in man," Lancet 278, 806-807 (1961).

40. B. L. Holman et al., "Regional cerebral blood flow with the Anger camera," J. Nucl. Med. 13, 916-923 (1972).

41. N. A. Lassen, "Blood flow of the cerebral cortex calculates from ${ }^{85}$ Kripton-beta-clearance recorded over the exposed surface; Evidence of inhomogeneity of flow," Acta Neur. Scand. 41, 24-28 (1965).

42. E. J. Hanson, R. E. Anderson, and T. M. Sundt, "Comparison of ${ }^{85}$ Kripton and ${ }^{133}$ Xenon cerebral blood flow measurements before, during, and following focal, incomplete ischemia in the squirrel monkey," Circ Res. 36, 18-26 (1975).

43. K. L. Zierler, "Equations for measuring blood flow by external monitoring of radioisotopes," Circ. Res. 16, 309-321 (1965).

44. R. Y. Z. Chen et al., "Tissue-blood partition coefficient for xenon: temperature and hematocrit dependence," J. Appl. Physiol. Respir. Environ. Exerc. Physiol. 49, 178-183 (1980).

45. N. A. Lassen et al., "Regional cerebral blood flow in man determined by krypton ${ }^{85}$," Neurology 13, 719-727 (1963).

46. J. Olesen, O. B. Paulson, and N. A. Lassen, "Regional cerebral blood flow in man determined by the initial slope of the clearance of intra-arterially injected ${ }^{133} \mathrm{Xe}$," Stroke 2, 519-540 (1971).

47. R. C. Walovitch et al., "Characterization of technetium-99m-L, L- ECD for brain perfusion imaging, part 1: pharmacology of technetium-99m ECD in nonhuman primates," J. Nucl. Med. 30(11), 1892-1901 (1989).

48. J. Léveillé et al., "Characterization of technetium-99m-L, L-ECD for brain perfusion imaging, part 2: biodistribution and brain imaging in humans," J. Nucl. Med. 30, 1902-1910 (1989).

49. N. A. Lassen et al., "The retention of [99mTc]-d, 1-HM-PAO in the human brain after intracarotid bolus injection: a kinetic analysis," J. Cereb. Blood Flow Metab. 8(6), S13-S22 (1988).

50. K. Ishizu et al., "Extraction and retention of technetium-99m-ECD in human brain: dynamic SPECT and oxygen-15-water PET studies," J. Nucl. Med. 37, 1600-1604 (1996).

51. H. Matsuda et al., "Acetazolamide effect on vascular response in areas with diaschisis as measured by Tc-99m HMPAO brain SPECT," Clin. Nucl. Med. 17(7), 581-586 (1992).

52. S. Asenbaum et al., "Imaging of cerebral blood flow with technetium99m-HMPAO and technetium-99m-ECD: a comparison," J. Nucl. Med. 39(4), 613-618 (1998).

53. P. Herscovitch, J. Markham, and M. E. Raichle, "Brain blood flow measured with intravenous H2(15)O. I. Theory and error analysis," J. Nucl. Med. 24, 782-789 (1983).

54. M. E. Raichle et al., "Brain blood flow measured with intravenous H2 (15)O. II. Implementation and validation," J. Nucl. Med. 24, 790-798 (1983).

55. O. Carlsen and O. Hedegaard, "Evaluation of regional cerebral circulation based on absolute mean transit times in radionuclide cerebral angiography," Phys. Med. Biol. 32, 1457-1467 (1987).

56. M. E. Raichle, "Quantitative in vivo autoradiogaphy with positron emission tomography," Brain Res. Rev. 1, 47-68 (1979).

57. W. J. Powers et al., "Cerebral blood flow and cerebral metabolic rate of oxygen requirements for cerebral function and viability in humans," J. Cereb. Blood Flow Metab. 5, 600-608 (1985).

58. J. A. K. Blokland et al., "Positron emission tomography: a technical introduction for clinicians," Eur. J. Radiol. 44(1), 70-75 (2002).

59. D. W. Johnson et al., "Stable xenon CT cerebral blood flow imaging: rationale for and role in clinical decision making," AJNR 12, 201-213 (1991).

60. A. P. Carlson et al., "Xenon-enhanced cerebral blood flow at $28 \%$ xenon provides uniquely safe access to quantitative, clinically useful cerebral blood flow information: a multicenter study," AJNR 32(7), 1315-1320 (2011).

61. E. Rostami et al., "Monitoring of cerebral blood flow and metabolism bedside in patients with subarachnoid hemorrhage - a Xenon-CT and microdialysis study," Front. Neurol. 5(June), 89 (2014).

62. E. G. Hoeffner et al., "Cerebral perfusion CT: technique and clinical applications," Radiology 231, 632-644 (2004).
63. B. Turowski and P. Schramm, "An appeal to standardize CT- and MR-perfusion," Clin. Neuroradiol. 25(Suppl 2), 205-210 (2015).

64. R. E. Latchaw et al., "Guidelines and recommendations for perfusion imaging in cerebral ischemia," Stroke 34(4), 1084-1104 (2003).

65. D. V. Sahani, "Perfusion CT: an overview of technique and clinical applications," in Int. Society for Magnetic Resonance in Medicine, 18th Scientific Meeting \& Exhibition and the Society for Magnetic Resonance Technologists, Vol. 18 (2010).

66. H. C. Roberts, "Neuromaging techniques in cerebrovascular disease: computed tomography angiography/computed tomography perfusion," Semin. Cerebrovasc. Dis. Stroke 1(4), 303-316 (2001).

67. M. Wintermark et al., "Vasospasm after subarachnoid hemorrhage: utility of perfusion CT and CT angiography on diagnosis and management," AJNR 27(1), 26-34 (2006).

68. W. W. Orrison et al., "Whole-brain dynamic CT angiography and perfusion imaging," Clin. Radiol. 66(6), 566-574 (2011).

69. A. Villringer et al., "Dynamic imaging with lanthanide chelates in normal brain: contrast due to magnetic susceptibility effects," Magn. Reson. Med. 6, 164-174 (1988).

70. L. Østergaard et al., "High resolution measurement of cerebral blood flow using intravascular tracer bolus passages. Part I: mathematical approach and statistical analysis," Magn. Res. Med. 36, 715-725 (1996).

71. F. Calamante et al., "Measuring cerebral blood flow using magnetic resonance imaging techniques," J. Cereb. Blood Flow Metab. 19, 701-735 (1999).

72. L. Østergaard, "Principles of cerebral perfusion imaging by bolus tracking," J. Magn. Res. Imag. 22, 710-717 (2005).

73. T.-Q. Li et al., "Quantification of cerebral blood flow by bolus tracking and artery spin tagging methods," Magn. Res. Imag. 18, 503-512 (2000).

74. M. Essig et al., "Perfusion MRI: the five most frequently asked technical questions," Am. J. Roentgenol. 200, 24-34 (2013).

75. P. Korfiatis and B. Erickson, "The basics of diffusion and perfusion imaging in brain tumors," Appl. Rad. 43, 22-29 (2014).

76. J. A. Detre et al., "Perfusion imaging," Magn. Res. Med. 23, 37-45 (1992).

77. D. S. Williams et al., "Magnetic resonance imaging of perfusion using spin inversion of arterial water," Proc. Natl. Acad. Sci. USA 89, 212216 (1992).

78. J. A. Detre and D. C. Alsop, "Perfusion magnetic resonance imaging with continuous arterial spin labeling: methods and clinical applications in the central nervous system," Eur. J. Rad. 30, 115-124 (1999).

79. X. Golay, J. Hendrikse, and T. C. C. Lim, "Perfusion imaging using arterial spin labeling," Top. Magn. Reson. Imaging 15, 10-27 (2004).

80. R. R. Edelman et al., "Qualitative mapping of cerebral blood flow and functional localization with echo-planar MR imaging and signal targeting with alternating radio frequency (STAR) sequences: applications to MR angiography," Radiology 192, 513-520 (1994).

81. E. C. Wong, R. B. Buxton, and L. R. Frank, "Implementation of quantitative perfusion imaging techniques for functional brain mapping using pulsed arterial spin labeling," NMR Biomed. 10, 237-249 (1997).

82. S.- G. Kim, "Quantification of regional blood flow change by flowsensitive alternating inversion recovery (FAIR) technique: application to functional mapping," Magn. Reson. Med. 37, 425-435 (1995).

83. E. C. Wong, R. B. Buxton, and L. R. Frank, "Quantitative imaging of perfusion using a single subtraction (QUIPSS and QUIPSS II)," Magn. Reson. Med. 39, 702-708 (1998).

84. R. Aaslid, T. M. Markwalder, and H. Nornes, "Noninvasive transcranial Doppler ultrasound recording of flow velocity in basal cerebral arteries," J. Neurosurg. 57, 769-774 (1982).

85. J. Lu, K. A. Mamum, and T. Chau, "Online transcranial Doppler ultrasonographic control of an onscreen keyboard," Front. Hum. Neurosci. 8, 199 (2014).

86. N. B. Smith and A. Webb, Introduction to Medical Imaging: Physics, Engineering and Clinical Applications, Cambridge University Press, Cambridge, UK (2011).

87. U. Bogdahn et al., "Transcranial color-coded real-time sonography in adults," Stroke 21, 1680-1688 (1990).

88. M. L. Sanderson and H. Yeung, "Guidelines for the use of ultrasonic non-invasive metering techniques," Flow Meas. Instrum. 13, 125-142 (2002). 
89. F. T. Charbel et al., "Quantitative assessment of vessel flow integrity for aneurysm surgery," J. Neurosurg. 91, 1050-1054 (1999).

90. P. Le Roux, "Invasive neurological and multimodality monitoring in the neuroICU," in Textbook of Neurointensive Care, A. J. Layon, A. Gabrielli, and W. A. Friedman, Eds., pp. 127-145, Springer, London (2013).

91. A. Bhatia and A. K. Gupta, "Neuromonitoring in the intensive care unit. I. Intracranial pressure and cerebral blood flow monitoring," Intensive Care Med. 33(7), 1263-1271 (2007).

92. G. T. Martin and H. F. Bowman, "Validation of real-time continuous perfusion measurement," Med. Biol. Eng. Comput. 38, 319-325 (2000).

93. P. Vajkoczy et al., "Continuous monitoring of regional cerebral blood flow: experimental and clinical validation of a novel thermal diffusion microprobe," J. Neurosurg. 93, 265-274 (2000).

94. J. W. Valvano, J. T. Allen, and H. F. Bowman, "The simultaneous measurement of thermal conductivity, thermal diffusivity, and perfusion in small volumes of tissue," J. Biomech. Eng. 106(3), 192-197 (1984).

95. C. N. Seubert, J. E. Cibula, and M. E. Mahla, "Noninvasive monitoring in the neurointensive care unit: EEG, oximetry, TCD," in Textbook of Neurointensive Care, A. J. Layon, A. Gabrielli, and W. A. Friedman, Eds., pp. 109-126, Springer, London (2013).

96. G. Rosenthal et al., "Incorporating a parenchymal thermal diffusion cerebral blood flow probe in bedside assessment of cerebral autoregulation and vasoreactivity in patients with severe traumatic brain injury," J. Neurosurg. 114(1), 62-70 (2011).

97. M. D. Stern, "In vivo evaluation of microcirculation by coherent light scattering," Nature 254, 56-58 (1975).

98. U. Dirnagl et al., "Continuous measurement of cerebral cortical blood flow by laser-Doppler flowmetry in a rat stroke model," J. Cereb. Blood Flow Metab. 9, 589-596 (1989).

99. I. Fredriksson, C. Fors, and J. Johansson, "Laser doppler flowmetry - a theoretical framework," Department of Biomedical Engineering, Linköping University (2007), www.imt.liu.se/bit/ldf/ldfmain.html (20 December 2015).

100. T. Durduran, "Non-invasive measurements of tissue hemodynamics with hybrid diffuse optical methods," PhD Thesis, University of Pennsylvania, Philadelphia, PA (2004).

101. P. J. Kirkpatrick et al., "Continuous monitoring of cortical perfusion by laser Doppler flowmetry in ventilated patients with head injury," J. Neurol. Neurosurg. Psychiatry 57(11), 1382-1388 (1994).

102. B. A. Sutherland, T. Rabie, and A. M. Buchan, "Laser Doppler flowmetry to measure changes in cerebral blood flow," in Cerebral Angiogenesis: Methods and Protocols, Vol. 1135, R. Milner, Ed., pp. 237-248, Springer, New York, NY (2014).

103. T. Durduran and A. G. Yodh, "Diffuse correlation spectroscopy for non-invasive, micro-vascular cerebral blood flow measurement," NeuroImage 85, 51-63 (2014).

104. E. M. Buckley et al., "Diffuse correlation spectroscopy for measurement of cerebral blood flow: future prospects," Neurophotonics 1(1), 011009 (2014).

105. A. D. Boas and A. G. Yodh, "Spatially varying dynamical properties of turbid media probed with diffusing temporal light correlation," J. Opt. Soc. Am. A 14, 192-215 (1997).

106. R. C. Mesquita et al., "Influence of probe pressure on the diffuse correlation spectroscopy blood flow signal: extra-cerebral contributions," Biomed. Opt. Express 4(7), 978-994 (2013).

107. M. Ferrari and V. Quaresima, "A brief review on the history of human functional near-infrared spectroscopy (fNIRS) development and fields of application," NeuroImage 63, 921-935 (2012).

108. F. Scholkmann et al., "A review on continuous wave functional nearinfrared spectroscopy and imaging instrumentation and methodology," NeuroImage 85, 6-27 (2014).

109. A. Torricelli et al., "Time domain functional NIRS imaging for human brain mapping," Neurolmage 85, 28-50 (2014).

110. M. Smith, "Shedding light on the adult brain: a review of the clinical applications of near-infrared spectroscopy," Phil. Trans. R. Soc. A 369, 4452-4469 (2011).

111. A. Casati et al., "Continuous monitoring of cerebral oxygen saturation in elderly patients undergoing major abdominal surgery minimizes brain exposure to potential hypoxia," Anesth. Analg. 101, 740-747 (2007).
112. M. M. Tisdall et al., "Increase in cerebral aerobic metabolism by normobaric hyperoxia after traumatic brain injury," J. Neurosurg. 109, 424-432 (2008).

113. S. R. Leal-Noval et al., "Invasive and noninvasive assessment of cerebral oxygenation in patients with severe traumatic brain injury," Intens. Care Med. 36, 1309-1317 (2010).

114. F. Fabbri et al., "Bilateral near-infrared monitoring of the cerebral concentration and oxygen saturation of hemoglobin during right unilateral electro-convulsive therapy," Brain Res. 992, 193-204 (2003).

115. M. Tsuji et al., "Near infrared spectroscopy detects cerebral ischemia during hypotension in piglets," Pediatr. Res. 44, 591-595 (1998).

116. J. S. Soul et al., "Noninvasive detection of changes in cerebral blood flow by near-infrared spectroscopy in a piglet model of hydrocephalus," Pediatr. Res. 48(4), 445-449 (2000).

117. H. Bassan et al., "Identification of pressure passive cerebral perfusion and its mediators after infant cardiac surgery," Pediatr. Res. 57(1), 35-41 (2005).

118. B. P. Wagner et al., "Rapid assessment of cerebral autoregulation by near-infrared spectroscopy and a single dose of phenylephrine," Pediatr. Res. 69(5 Pt 1), 436-441 (2011).

119. A. D. Edwards et al., "Cotside measurement of cerebral blood flow in ill newborn infants by near infrared spectroscopy," Lancet 332, 770771 (1988).

120. C. E. Elwell et al., "Quantification of adult cerebral hemodynamics by near-infrared spectroscopy," J. Appl. Physiol. 77(6), 2753-2760 (1994).

121. P. W. McCormick et al., "Noninvasive cerebral optical spectroscopy for monitoring cerebral oxygen delivery and hemodynamics," Crit. Care Med. 19, 89-97 (1991).

122. I. Roberts et al., "Estimation of cerebral blood flow with near infrared spectroscopy and indocyanine green," Lancet 342, 1425 (1993).

123. D. W. Brown et al., "Quantitative near infrared spectroscopy measurement of cerebral hemodynamics in newborn piglets," Pediatr. Res. 51(5), 564-570 (2002).

124. J. A. Cooper et al., "Continuous monitoring of absolute cerebral blood flow by near-infrared spectroscopy during global and focal temporary vessel occlusion," J. Appl. Physiol. 110(6), 1691-1698 (2011).

125. K. Verdecchia et al., "Quantifying the cerebral metabolic rate of oxygen by combining diffuse correlation spectroscopy and time-resolved near-infrared spectroscopy," J. Biomed. Opt. 18(2), 027007 (2013).

126. T. S. Leung et al., "Theoretical investigation of measuring cerebral blood flow in the adult human head using bolus Indocyanine Green injection and near-infrared spectroscopy," Appl. Opt. 46(10), 1604 (2007).

127. S. Fantini, "Dynamic model for the tissue concentration and oxygen saturation of hemoglobin in relation to blood volume, flow velocity, and oxygen consumption: implications for functional neuroimaging and coherent hemodynamics spectroscopy (CHS)," Neurolmage $\mathbf{8 5}$, 202-221, (2014).

128. S. Fantini et al., "Non-invasive assessment of cerebral microcirculation with diffuse optical and coherent hemodynamics spectroscopy," Proc. SPIE 9690, 96900S (2016).

129. M. L. Pierro et al., "Validation of a novel hemodynamic model for coherent hemodynamics spectroscopy (CHS) and functional brain studies with fNIRS and fMRI," Neurolmage 85, 222-233 (2014).

130. J. M. Kainerstorfer, A. Sassaroli, and S. Fantini, "Coherent hemodynamics spectroscopy in a single step," Biomed. Opt. Express 5(10), 3403-3616 (2014).

131. J. M. Kainerstorfer et al., "Practical steps for applying a new dynamic model to near-infrared spectroscopy measurements of hemodynamic oscillations and transient changes: implications for cerebrovascular and functional brain studies," Acad. Radiol. 21(2), 185-196 (2014).

132. S. Fantini, "A new hemodynamic model shows that temporal perturbations of cerebral blood flow and metabolic rate of oxygen cannot be measured individually using functional near-infrared spectroscopy.," Physiol. Meas. 35(1), N1-N9 (2014).

133. M. L. Pierro et al., "Reduced speed of microvascular blood flow in hemodialysis patients versus healthy controls: a coherent hemodynamics spectroscopy study," J. Biomed. Opt. 19(2), 026005 (2014).

134. ATOTW (Anesthesia Tutorial of The Week) n. 137, "AnaesthesiaUK," http://www.anaesthesiauk.com/Documents/137\%20Physical\%20principles $\% 20$ of\%20intra-arterial\%20blood\%20pressure\%20measurement.pdf (June 8 2009). 
135. I. Moxham, "Physics of invasive blood pressure monitoring," South. Afr. J. Anaesthesia Analgesia 9, 33-38 (2003).

136. R. M. Gardner, "Direct blood pressure measurement - dynamic response requirements," Anesthesiology 54, 227-236 (1981).

137. B. P. M. Imholz et al., "Fifteen years experience with finger arterial pressure monitoring: assessment of the technology," Cardiovasc. Res. 38, 605-616 (1998).

138. K. H. Wesseling et al., "Physiocal, calibrating finger vascular physiology for Finapres," Homeostasis 36, 67-82 (1995).

139. L. Peter et al., "A review of methods for non-invasive and continuous blood pressure monitoring: pulse transit time method is promising?," IRBM 35, 271-282 (2014)

140. G. Beevers et al., "Blood pressure measurement. Part II—conventional sphygmomanometry: technique of auscultatory blood pressure measurement," BMJ 322, 1043-1047 (2001).

141. G. Ogedegbe and T. Pickering, "Principles and techniques of blood pressure measurement," Cardiol. Clin. 28, 571-586 (2010).

142. T. Dieterle, "Blood pressure measurement - an overview," Swiss Med. Weekly 142, 1-9 (2012).

143. M. R. Nelson et al., "Noninvasive measurement of central vascular pressures with arterial tonometry: clinical revival of the pulse pressure waveform?," Mayo Clin. Proc. 85, 460-472 (2010).

144. G. Parati et al., "Comparison of finger and intra-arterial blood pressure monitoring at rest and during laboratory testing," Hypertension 13, 647-655 (1989).

145. T. Sato et al., "Accuracy of a continuous blood pressure monitor based on arterial tonometry," Hypertension 21, 866-874 (1993).

146. L. A. Steiner et al., "Assessment of cerebrovascular autoregulation in head-injured patients. A validation study," Stroke 34, 2404-2409 (2003).

147. A. R. Zazulia et al., "Autoregulation of cerebral blood flow to changes in arterial pressure in mild Alzheimer's disease," J. Cerebral Blood Flow Metab. 30, 1883-1889 (2010).

148. J. F. Schmidt et al., "Computerized analysis of cerebral blood flow autoregulation in humans: validation of a method for pharmacological studies," J. Cardiovascular Pharmacol. 15, 983-988 (1990).

149. M. A. Horsfield et al., "Regional differences in dynamic cerebral autoregulation in the healthy brain assessed by magnetic resonance imaging," PLoS One 8, e62588 (2013).

150. M. J. de Boorder, J. Hendrikse, and J. van der Grond, "Phase-contrast magnetic resonance imaging measurements of cerebral autoregulation with a breath-hold challenge. A feasibility study," Stroke 35, 13501354 (2004).

151. M. Tsuji et al., "Cerebral intravascular oxygenation correlates with mean arterial pressure in critically ill premature infants," Pediatrics 106, 625-632 (2000).

152. B. P. Wagner and J. Pfenninger, "Dynamic cerebral autoregulatory response to blood pressure rise measured by near-infrared spectroscopy and intracranial pressure," Crit. Care Med. 30, 2014-2021 (2002).

153. K. M. Brady et al., "Continuous time-domain analysis of cerebrovascular autoregulation using near-infrared spectroscopy," Stroke 38, 2818-2825 (2007)

154. J. S. Soul et al., "Fluctuating pressure-passivity is common in the cerebral circulation of sick premature infants," Pediatr. Res. 61, 467-473 (2007).

155. F. Y. Wong et al., "Impaired autoregulation in preterm infants identified by using spatially resolved spectroscopy," Pediatrics 121, e604e611 (2008).

156. J. K. Lee et al., "Cerebrovascular reactivity measured by near-infrared spectroscopy," Stroke 40, 1820-1826 (2009).

157. P. Nissen et al., "Near-infrared spectroscopy for evaluation of cerebral autoregulation during orthotopic liver transplantation," Neurocrit. Care 11, 235-241 (2009).

158. L. A. Steiner et al., "Near-infrared spectroscopy can monitor dynamic cerebral autoregulation in adults," Neurocrit. Care 10, 122-128 (2009).

159. C. Zweifel et al., "Continuous assessment of cerebral autoregulation with near-infrared spectroscopy in adults after subarachnoid hemorrhage," Stroke 41(9), 1963-1968 (2010).

160. G. H. Hahn et al., "Applicability of near-infrared spectroscopy to measure cerebral autoregulation noninvasively in neonates: a validation study in piglets," Pediatr. Res. 70 166-170 (2011).
161. M. Reinhard et al., "Spatial mapping of dynamic cerebral autoregulation by multichannel nearinfrared spectroscopy in high-grade carotid artery disease," J. Biomed. Opt. 19, 097005 (2014).

162. V. J. Burton et al., "A pilot cohort study of cerebral autoregulation and 2 -year neurodevelopmental outcomes in neonates with hypoxicischemic encephalopathy who received therapeutic hypothermia," BMC Neurol. 15, 209 (2015).

163. R. M. G. Berg and R. R. Plovsing, "Near-infrared spectroscopy versus transcranial Doppler ultrasound for assessing dynamic cerebral autoregulation by transfer function analysis in sepsis," Scand. J. Clin. Lab. Inv. 76(1), 88-91 (2016).

164. D. Hori et al., "Cerebral autoregulation monitoring with ultrasoundtagged near-infrared spectroscopy in cardiac surgery patients," Anesth. Analg. 121, 1187-1193 (2015).

165. A. Tsalach et al., "Depth selective acousto-optic flow measurement," Biomed. Opt. Express 6, 4871-4886 (2015).

166. J. M. Kainerstorfer et al., "Cerebral autoregulation in the microvasculature measured with near-infrared spectroscopy," J. Cerebral Blood Flow Metabo. 35, 959-966 (2015).

167. J. Kolodjaschna et al., "Comparison of autoregulatory mechanisms between middle cerebral artery and ophthalmic artery after thigh cuff deflation in healthy subjects," IOVS 46, 636-640 (2005).

168. S. K. Kirkham, R. E. Craine, and A. A. Birch, "A new mathematical model of dynamic cerebral autoregulation based on a flow dependent feedback mechanism," Physiol. Meas. 22, 461-473 (2001).

169. M. S. Olufsen, A. Nadim, and L. A. Lipsitz, "Dynamics of cerebral blood flow regulation explained using a lumped parameter model," Am. J. Physiol. Regul. Integr. Comp Physiol. 282, R611-R622 (2002).

170. M. Czosnyka et al., "Contribution of mathematical modelling to the interpretation of bedside tests of cerebrovascular autoregulation," J. Neurol. Neurosurg. Psych. 63, 721-731 (1997).

171. M. Ursino, M. Iezzi, and N. Stocchetti, "Intracranial pressure dynamics in patients with acute brain damage: a critical analysis with the aid of a mathematical model," IEEE Trans. Biomed. Eng. 42, 529-540 (1995).

172. M. Ursino and C. A. Lodi, "A simple mathematical model of the interaction between intracranial pressure and cerebral hemodynamics," J. Appl. Physiol. 82, 1256-1269 (1997).

173. M. Ursino and C. A. Lodi, "Interaction among autoregulation, $\mathrm{CO}_{2}$ reactivity, and intracranial pressure: a mathematical model," Am. J. Physiol. Heart Circ. Physiol. 274, H1715-H1728 (1998).

174. M. Ursino et al., "Cerebral hemodynamics during arterial and $\mathrm{CO}_{2}$ pressure changes: in vivo prediction by a mathematical model," Am. J. Physiol. Heart Circ. Physiol. 279, H2439-H2455 (2000).

175. S. J. Payne and L. Tarassenko, "Combined transfer function analysis and modelling of cerebral autoregulation," Ann. Biomed. Eng. 34, $847-858$ (2006).

176. M. Banaji et al., "A physiological model of cerebral blood flow control," Math. Biosci. 194, 125-173 (2005).

177. M. Banaji et al., "A model of brain circulation and metabolism: NIRS signal changes during physiological challenges," PLoS Comp. Biol. 4, e1000212 (2008).

178. M. Caldwell et al., "BrainSignals revisited; simplifying a computational model of cerebral physiology," PLOS ONE 10, e0126695 (2015).

179. M. Ursino and P. Di Giammarco, "A mathematical model of the relationship between cerebral blood volume and intracranial pressure changes: the generation of plateau waves," Ann. Biomed. Eng. 19, 15-42 (1991).

180. A. Sassaroli, J. M. Kainerstorfer, and S. Fantini, "Nonlinear extension of a hemodynamic linear model for coherent hemodynamics spectroscopy," J. Theor. Biol. 389, 132-145 (2016).

181. J. M. K. Lam, J. N. K. Hsiang, and W. S. Poon, "Monitoring of autoregulation using laser Doppler flowmetry in patients with head injury," J. Neurosurg. 86 438-445 (1997).

182. F. P. Tiecks et al., "Comparison of static and dynamic cerebral autoregulation measurements" Stroke 26, 1014-1019 (1995).

183. R. Zhang et al., "Transfer function analysis of dynamic cerebral autoregulation in humans," Am. J. Physiol. Heart Circ. Physiol. 274, H233H241 (1998).

184. R. R. Diehl et al., "Phase relationship between cerebral blood flow velocity and blood pressure," Clin. Test Autoregul. Stroke 26, 1801-1804 (1995). 
185. A. A. Birch et al., "Assessment of autoregulation by means of periodic changes in blood pressure," Stroke 26, 834-837 (1995).

186. Y. C. Tzeng et al., "Determinants of human cerebral pressure-flow velocity relationships: new insights from vascular modelling and $\mathrm{Ca}^{2+}$ channel blockade," J. Physiol. 589, 3263-3274 (2011).

187. C. A. Giller, "The frequency-dependent behavior of cerebral autoregulation," Neurosurgery 27, 362-368 (1990).

188. R. B. Panerai et al., "Cerebral autoregulation dynamics in premature newborns," Stroke 26, 74-80 (1995).

189. R. B. Panerai et al., "Analysis of cerebral blood flow autoregulation in neonates," IEEE Trans. Biomed. Eng. 43, 779-788 (1996).

190. R. B. Panerai, A. W. R. Kelsall, and J. M. Rennie, "Model selection for pressure-flow velocity relationships of cerebral arteries," in IEEE Proc. Computer Cardiology, pp. 779-782 (1993).

191. R. B. Panerai, S. L. Dawson, and J. F. Potter, "Linear and nonlinear analysis of human dynamic cerebral autoregulation," Am. J. Physiol. Heart Circ. Physiol. 277, H1089-H1099 (1999).

192. G. D. Mitsis et al., "Nonlinear modeling of the dynamic effects of arterial pressure and $\mathrm{CO}_{2}$ variations on cerebral blood flow in healthy humans," IEEE Trans Biomed Eng. 51, 1932-1943 (2004).

193. M. Latka et al., "Phase dynamics in cerebral autoregulation," Am. J. Physiol. Heart Circ. Physiol. 289, H2272-H2279 (2005).

194. V. Novak et al., "Multimodal pressure-flow method to assess dynamics of cerebral autoregulation in stroke and hypertension," Biomed Eng. Online 3, 39 (2004).

195. R. D. Bell and B. V. Zlokovic, "Neurovascular mechanisms and blood-brain barrier disorder in Alzheimer's disease," Acta. Neuropathol. 118(1), 103-113 (2009).

196. C. E. Wierenga, C. C. Hays, and Z. Z. Zlatar, "Cerebral blood flow measured by arterial spin labeling MRI as a preclinical marker of Alzheimer's disease," J. Alzheimers Dis. 42(Suppl 4), S411-S419 (2014).

197. V. Valotassiou et al., "Clinical evaluation of brain perfusion SPECT with Brodmann areas mapping in early diagnosis of Alzheimer's disease," J. Alzheimers Dis. 47(3), 773-785 (2015).

198. T. Araki et al., "The effects of combine treatment of memantine and donepezil on Alzheimer's disease patients and its relationship with cerebral blood flow in the prefrontal area," Int. J. Geriatr. Psychiatry 29(9), 881-889 (2014).

199. X. Chen et al., "Donepezil effects on cerebral blood flow in older adults with mild cognitive deficits," J. Neuropsychiatry Clin. Neurosci. 18(2), 178-185 (2006).

200. K. Imamura, N. Okayasu, and T. Nagatsu, "Cerebral blood flow and freezing of gait in Parkinson's disease," Acta. Neurol. Scand. 126(3), 210-218 (2012).

201. J. J. Sidtis et al., "Therapeutic high-frequency stimulation of the subthalamic nucleus in Parkinson's disease produces global increases in cerebral blood flow," J. Cereb. Blood Flow Metab. 32(1), 41-49 (2012).

202. E. Indelicato et al., "Cerebral autoregulation and white matter lesions in Parkinson's disease and multiple system atrophy," Parkinsonism Relat. Disord. 21(12), 1393-1397 (2015).

203. A. M. Brickman et al., "Cerebral autoregulation, beta amyloid, and white matter hyperintensities are interrelated," Neurosci. Lett. 592, 54-58 (2015).

204. S. S. Kety and R. B. Woodford, "Cerebral blood flow and metabolism in schizophrenia: the effects of barbiturate semi-narcosis, insulin coma and electroshock," Am. J. Psychiatry 104 (12), 765-770 (1948).

205. A. Pinkham et al., "Resting quantitative cerebral blood flow in schizophrenia measured by pulsed arterial spin labeling perfusion MRI," Psychiatry Res 194(1), 64-72 (2011).

206. O. Sabri et al., "Correlation of positive symptoms exclusively to hyperperfusion or hypoperfusion of cerebral cortex in never-treated schizophrenics," Lancet 349(9067), 1735-1739 (1997).

207. N. Agarwal et al., "Increased fronto-temporal perfusion in bipolar disorder," J. Affect. Disord. 110, 106-114 (2008).

208. N. Vasic et al., "Baseline brain perfusion and brain structure in patients with major depression: a multimodal magnetic resonance imaging study," J. Psychiatry Neurosci. 40(6), 412-421 (2015).

209. Y. Monden et al., "Clinically-oriented monitoring of acute effects of methylphenidate on cerebral hemodynamics in ADHD children using fNIRS," Clin. Neurophysiol. 123(6), 1147-1157 (2012).
210. J. H. Shin et al., "Using relative cerebral blood flow and volume to evaluate the histopathologic grade of cerebral gliomas: preliminary results," AJR Am. J. Roentgenol. 179(3), 783-789 (2002).

211. H. Cebeci et al., "Assesment of perfusion in glial tumors with arterial spin labeling; comparison with dynamic susceptibility contrast method," Eur. J. Radiol. 83(10), 1914-1919 (2014).

212. O. Togao et al., "Differentiation of high-grade and low-grade diffuse gliomas by intravoxel incoherent motion MR imaging," Neuro. Oncol. 18(1), 132-141 (2016).

213. K. Ouriel et al., "Intracerebral hemorrhage after carotid endarterectomy: incidence, contribution to neurologic morbidity, and predictive factors," J. Vasc. Surg. 29(1), 82-89 (1999).

214. K. Hosoda et al., "Cerebral vasoreactivity and internal carotid artery flow help to identify patients at risk for hyperperfusion after carotid endarterectomy," Stroke 32(7), 1567-1573 (2001).

215. N. Komoribayashi et al., "Cerebral hyperperfusion after carotid endarterectomy is associated with preoperative hemodynamic impairment and intraoperative cerebral ischemia," J. Cereb. Blood Flow Metab. 26(7), 878-884 (2006).

216. N. Hecht et al., "Laser speckle imaging allows real-time intraoperative blood flow assessment during neurosurgical procedures," J. Cereb. Blood Flow Metab. 33(7), 1000-1007 (2013).

217. N. Hecht et al., "Intraoperative monitoring of cerebral blood flow by laser speckle contrast analysis," Neurosurg. Focus 27(4), E11 (2009).

218. R. M. Chesnut et al., "A trial of intracranial-pressure monitoring in traumatic brain injury," $N$. Engl. J. Med. 367(26), 2471-2481 (2012).

219. D. W. Marion, J. Darby, and H. Yonas, "Acute regional cerebral blood flow changes caused by severe head injuries," J. Neurosurg. 74(3), 407-414 (1991).

220. P. J. D. Andrews et al., "NICEM consensus on neurological monitoring in acute neurological disease," Intensive Care Med. 34(8), 1362-1370 (2008).

221. E. Rostami, H. Engquist, and P. Enblad, "Imaging of cerebral blood flow in patients with severe traumatic brain injury in the neurointensive care," Front. Neurol. 5(July), 1-9 (2014).

222. G. Granata et al., "Posterior reversible encephalopathy syndromeinsight into pathogenesis, clinical variants and treatment approaches," Autoimmun. Rev.14(9), 830-836 (2015).

223. C. Lamy, C. Oppenheim, and J. L. Mas, "Posterior reversible encephalopathy syndrome," Handb. Clin. Neurol. 121, 1687-1701 (2014).

224. G. Kumar, R.B. Shahripour, and M.R. Harrigan, "Vasospasm on transcranial Doppler is predictive of delayed cerebral ischemia in aneurysmal subarachnoid hemorrhage: a systematic review and metaanalysis," J. Neurosurg. 124, 1257-1264 (2015).

225. L. Calviere et al., "Prediction of delayed cerebral ischemia after subarachnoid hemorrhage using cerebral blood flow velocities and cerebral autoregulation assessment," Neurocrit. Care 23(2), 253-258 (2015).

226. V. Malinova et al., "Early whole-brain CT perfusion for detection of patients at risk for delayed cerebral ischemia after subarachnoid hemorrhage," J. Neurosurg. 1-9 (2015).

227. S. C. Zygmunt and T. J. Delgado-Zygmunt, "The haemodynamic effect of transcranial Doppler-guided high-dose nimodipine treatment in established vasospasm after subarachnoid haemorrhage," Acta. Neurochir. 135, 179-185 (1995).

228. L. da Costa et al., "Early identification of brain tissue at risk for delayed cerebral ischemia after aneurysmal subarachnoid hemorrhage," Acta. Neurochir. Suppl. 120, 105-109 (2015).

229. K. P Budohoski et al., "Cerebral autoregulation after subarachnoid hemorrhage: comparison of three methods," J. Cereb. Blood Flow Metab. 33(3), 449-456 (2013).

230. P. G. Al-Rawi and P. J. Kirkpatrick, "Tissue oxygen index: thresholds for cerebral ischemia using near-infrared spectroscopy," Stroke 37(11), 2720-2725 (2006).

231. Brain Trauma Foundation, American Association of Neurological Surgeons, and Congress of Neurological Surgeons, "Guidelines for the management of severe traumatic brain injury," J. Neurotrauma 24(Suppl 1), S1-S106 (2007).

232. G. J. Bouma et al., "Blood pressure and intracranial pressure-volume dynamics in severe head injury: relationship with cerebral blood flow," J. Neurosurg. 77(1), 15-19 (1992). 
233. G. J. Bouma and J. P Muizelaar, "Cerebral blood flow, cerebral blood volume, and cerebrovascular reactivity after severe head injury," J. Neurotrauma 9(Suppl 1), S333-S348 (1992).

234. U. Johnson et al., "Should the neurointensive care management of traumatic brain injury patients be individualized according to autoregulation status and injury subtype?," Neurocrit. Care 21(2), 259-265 (2014).

235. C. Miller et al., "Monitoring of cerebral blood flow and ischemia in the critically ill," Neurocrit. Care 21(Suppl 2), S121-S128 (2014).

236. B.- S. Lin et al., "Evaluation of traumatic brain injury by optical technique," BMC Neurol. 15, 202 (2015).

237. P. Taussky et al., "Validation of frontal near-infrared spectroscopy as noninvasive bedside monitoring for regional cerebral blood flow in brain-injured patients," Neurosurg. Focus 32(2), E2 (2012).

238. S.-C. Lee, J.-F. Chen, and S.-T. Lee, "Continuous regional cerebral blood flow monitoring in the neurosurgical intensive care unit," J. Clin. Neurosci. 12(5), 520-523 (2005).

239. P. Minciotti, M. G. Ceravolo, and L. Provinciali, "Inter-examiner variability of transcranial Doppler procedure and reports: a multicenter survey," Ital. J. Neurol. Sci. 18(1), 21-30 (1997).

240. F. Proust et al., "Usefulness of transcranial color-coded sonography in the diagnosis of cerebral vasospasm," Stroke 30(5), 1091-1098 (1999).

241. M. N. McDonnell et al., "Transcranial Doppler ultrasound to assess cerebrovascular reactivity: reliability, reproducibility and effect of posture," Peer J. 1, e65 (2013).

242. S. J. Han et al., "The Presto 1000: a novel automated transcranial Doppler ultrasound system," J. Clin. Neurosci. 22(11), 1771-1775 (2015).

243. P. N. Salibi et al., "Lifetime attributable risk of cancer from CT among patients surviving severe traumatic brain injury," AJR Am. J. Roentgenol. 202(2), 397-400 (2014).

244. D. M. Yousem, "The economics of functional magnetic resonance imaging: clinical and research," Neuroimaging Clin. N. Am. 24(4), 717-724 (2014).

245. M. N. Kim et al., "Continuous optical monitoring of cerebral hemodynamics during head-of-bed manipulation in brain-injured adults," Neurocrit. Care 20(3), 443-453 (2014).
246. J.-R. Kuo et al., "Wireless near-infrared spectroscopy system for determining brain hemoglobin levels in laboratory animals," J. Neurosci. Methods 214(2), 204-209 (2013).

Sergio Fantini is professor of biomedical engineering and principal investigator of the "Diffuse Optical Imaging of Tissue Laboratory" (DOIT Lab) at Tufts University. The research in the DOIT Lab aims to develop noninvasive applications of diffuse optics to assess cerebral perfusion, detect breast cancer, and quantify skeletal muscle oxygenation. His research resulted in eleven patents and about two hundred scientific publications. He coauthored with Prof. Irving Bigio (Boston University) a textbook on "Quantitative Biomedical Optics."

Angelo Sassaroli received his $\mathrm{PhD}$ degree in physics from the University of Electro-Communications, Tokyo (Japan), in 2002. From July 2002 to August 2007, he was a research associate at Tufts University, Medford, Massachusetts, where he has been a research assistant professor since September 2007. He has authored or coauthored more than 70 peer reviewed publications in the areas of his interests which include diffuse optical imaging and near-IR spectroscopy.

Kristen T. Tgavalekos is a doctoral student in biomedical engineering at Tufts University. She completed her undergraduate training in electrical engineering at Northeastern University in 2013. Her research interests include medical imaging for disease detection and monitoring. Her current research focuses on using near-infrared spectroscopy for clinical monitoring of cerebral hemodynamics.

Joshua Kornbluth is an assistant professor of neurology and neurosurgery at the Tufts University School of Medicine. He is a board certified neurologist and neurointensivist and medical director of the Michael Neely Neurosciences Critical Care Unit. His clinical and research interests are in disorders of consciousness, traumatic brain injury, and noninvasive methods of quantifying cerebral physiology. 